Superstructures,

heat and momentum transport in inclined turbulent thermal convection of low-Prandtl-number fluids

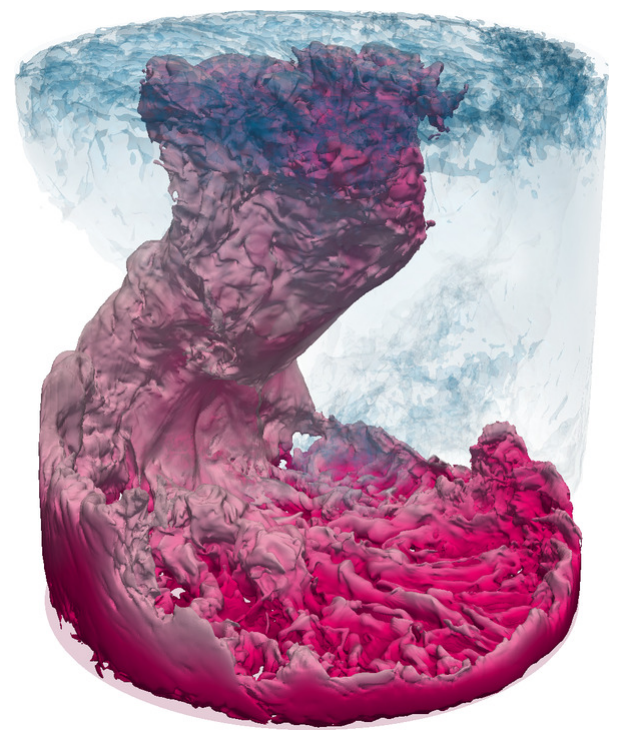

Lukas Zwirner 



\title{
Superstructures,
}

\section{heat and momentum transport in inclined turbulent thermal convection of low-Prandtl-number fluids}

\author{
Dissertation \\ for the award of the degree \\ "Doctor rerum naturalium" \\ Division of Mathematics and Natural Sciences \\ at the Georg-August-Universität Göttingen \\ within the doctoral degree program \\ Physics of Biological and Complex Systems \\ of the Göttingen Graduate School of Neurosciences, Biophysics, \\ and Molecular Biosciences (GGNB) \\ of the Georg-August University School of Sciences (GAUSS)
}

submitted by

Lukas Zwirner

from Neustadt am Rübenberge, Germany

Göttingen, 31.03.2020 


\section{Thesis Committee:}

\section{PD Dr. Olga Shishkina}

Department of Fluid Physics, Pattern Formation and Biocomplexity

Max Planck Institute for Dynamics and Self-Organization

\section{Prof. Dr. Andreas Tilgner}

Institute of Geophysics

Georg-August-University Göttingen

\section{Dr. Michael Wilczek}

Max Planck Research Group: Turbulence, Complex Flows \& Active Matter Max Planck Institute for Dynamics and Self-Organization

\section{Members of the Examination BoARD:}

PD Dr. Olga Shishkina (Referee)

Department of Fluid Physics, Pattern Formation and Biocomplexity

Max Planck Institute for Dynamics and Self-Organization

\section{Prof. Dr. Andreas Tilgner (Co-Referee)}

Institute of Geophysics

Georg-August-University Göttingen

\section{Other Members of the Examination BoARd:}

Dr. Michael Wilczek

Max Planck Research Group: Turbulence, Complex Flows \& Active Matter Max Planck Institute for Dynamics and Self-Organization

\section{Prof. Dr. Ulrich Parlitz}

Biomedical Physics Group

Max Planck Institute for Dynamics and Self-Organization

\section{Prof. Dr. Laurent Gizon}

Department Solar and Stellar Interiors

Max Planck Institute for Solar System Research

\section{Prof. Dr. Stefan Dreizler}

Institute of Astrophysics

Georg-August-University Göttingen 


\section{Abstract}

Recent experiments by Vasil'ev et al. (2015a) using liquid sodium as working fluid inside an extremely slender Rayleigh-Bénard convection (RBC) cell of the diameter-to-height aspect ratio $\Gamma=1 / 20$, which was inclined with respect to gravity, showed astonishing results: the heat transport through the system can be tenfold stronger for an inclination of $\beta \approx 65^{\circ}$, compared to classical RBC without inclination, $\beta=0^{\circ}$. As liquid metals are opaque and the flow structure is hardly accessible by experimental means, the main objective of this work is to conduct high-fidelity direct numerical simulations (DNS), where the full temperature and velocity fields are available, and to understand the complex relationship between the flow structures and the heat transport.

It is found that inside slender cylinders of $\Gamma=1 / 5$ at Prandtl numbers 1 and 0.1 , the heat transport is generally enhanced through inclination. This enhancement is related to a stronger single large-scale circulation (LSC) roll, which helps to create a hot and a cold plume column that eventually span the whole cylinder and impinge on the opposite boundary layer.

Furthermore, for low-Prandtl-number fluids $(\operatorname{Pr}=0.1)$ and slender cylinders $(\Gamma=1 / 5)$, the DNS reveal a global flow structure, that consists of up to four distinguishable rolls on top of each other. The heat transport is strongly determined by the number of rolls and decreases significantly as the number of rolls increases. Additionally, the elliptical instability is identified as the mechanism to break up the single-roll flow structure into multiple rolls.

Finally, DNS for $\Gamma=1$, extremely low Prandtl number $(\operatorname{Pr}=0.0094)$ and $R a=1.67 \times 10^{7}$ are directly compared to measurements in liquid sodium. The study demonstrates a quantitative agreement of the experimental and numerical results, in particular with respect to the global heat and momentum transport, temperature and velocity profiles, as well as the dynamics of the LSC. The DNS reveal that the twisting and sloshing of the LSC at small inclination angles periodically affects the instantaneous heat transport (with a variation of up to $\pm 44 \%$ of the mean heat transport). The twisted LSC is associated with a weak heat transport, while the sloshing mode that brings together the hot and cold streams of the LSC is associated with a strong heat transport. 



\section{Contents}

1 Introduction 1

2 Confined inclined thermal convection in low-Prandtl-number fluids 13

2.1 Introduction . . . . . . . . . . . . . . . . . . 13

2.2 Analytical relations and numerical simulations . . . . . . . . . . 17

2.2.1 Governing equations . . . . . . . . . . . . . . . . . 17

2.2.2 Exact relations for the dissipation rates in inclined convection 18

2.2.3 Direct numerical simulations and mesh resolution . . . . . . . 20

2.3 Results . . . . . . . . . . . . . . . . . . . . . . . . . 21

2.3.1 The effect of inclination . . . . . . . . . . . . 21

2.3.2 Plume columns and the flow structure of enhanced heat transfer 30

2.3.3 Single-roll and double-roll states in Rayleigh-Bénard convection 31

2.3.4 The effect of confinement . . . . . . . . . . . . . . . 36

2.3.5 Limiting cases: Rayleigh-Bénard and vertical convection . . . 39

2.4 Summary and conclusions . . . . . . . . . . . . . . . . . . . . . . 40

3 Elliptical instability and multi-roll flow modes of the large-scale circulation in confined turbulent Rayleigh-Bénard convection

3.1 Introduction . . . . . . . . . . . . . . . . . . . . . . . 43

3.2 Properties of different flow modes . . . . . . . . . . . . . . . . . 45

3.3 Mechanism of the mode transitions . . . . . . . . . . . . . . . . . . 49

3.4 Conclusions . . . . . . . . . . . . . . . . . . . . . . 50

3.5 Supplementary material: roll extraction algorithm . . . . . . . . . 50

4 The influence of the cell inclination on the heat transport and largescale circulation in liquid metal convection 53

4.1 Introduction . . . . . . . . . . . . . . . . . . 54

4.2 Methods . . . . . . . . . . . . . . . . . 60 60

4.2.1 Experiment . . . . . . . . . . . . . . . . 60

4.2 .2 Direct numerical simulations . . . . . . . . . . . 65

4.2 .3 Large-eddy Simulations . . . . . . . . . . . . . . . . 66

4.2.4 Methods of data analysis . . . . . . . . . . . . . . . . . 68

4.3 Results and discussion . . . . . . . . . . . . . . . . 72

4.3.1 Time-averaged heat and momentum transport . . . . . . . 75

4.3.2 Temperature and velocity profiles . . . . . . . . . . . . 82

4.3.3 Dynamics of the large-scale flow . . . . . . . . . . . . . . . 85

4.4 Conclusions . . . . . . . . . . . . . . . . . . . . . . . . 94

5 Summary \& Conclusions $\quad 97$

$\begin{array}{ll}\text { References } & 101\end{array}$ 



\section{Introduction}

Natural thermal convection is ubiquitous in nature and technical applications, thus it is deeply rooted in our day-to-day lives. As soon as we switch on the electric kettle in the morning to heat up some tea water, thermal convection occurs. We look outside the window and see thermal convection shaping our weather and climate, and over the horizon rises the sun. This distant source of energy and warmth for all mankind also relies on thermal convection.

As these examples show, thermal convection happens in different kinds of fluids, with various boundary conditions and often with additional physical effects. Buoyancy is the driving force behind thermal convection, and it arises due to the temperature-dependent density variation of the fluid. Studying a simple thermal convection system theoretically was initiated about 100 years ago by Lord Rayleigh (1916). He investigated the onset of thermal convection inside a fluid layer heated from below and cooled from above (cf. figure 1.1a). Bénard $(1900,1901)$ explored this kind of setup experimentally, and today it is named as "Rayleigh-Bénard convection (RBC)", acknowledging both of these fundamental contributions.

Since then, the Rayleigh-Bénard convection system has become one of the most thoroughly studied systems, however, there are still several open questions. Today's research interest on RBC devotes itself to the extremes: Extremely high Rayleigh numbers (thermal driving of the system) (Niemela et al., 2000), extremely low-Prandtl numbers (a fluid property) (Frick et al., 2015; Scheel \& Schumacher, 2017; Zürner et al., 2019), extremely diverse boundary conditions (for example roughness (Wagner \& Shishkina, 2015), very small (Vasil'ev et al., 2015a; Chong et al., 2015) or very wide aspect ratios (Stevens et al., 2018)). Furthermore, additional effects are considered, such as magnetic fields (Zhang \& Zikanov, 2014), rotation (Kunnen et al., 2011; Horn \& Shishkina, 2015; Zhang et al., 2020), double-diffusive systems (Garaud, 2018), non-Oberbeck-Boussinesq effects (Horn et al., 2013; Shishkina et al., 2016b) and moist convection (Prabhakaran et al., 2017), to name only a few. This work is also devoted to the extremes: well-resolved computer simulations of extremely low-Prandtl number fluids, i.e. liquid metals. Two additional effects are studied as well: the influence of the cell's inclination with respect to gravity and the influence of lateral confinement (small aspect ratio cells).

Before going to a more detailed introduction to set the present work into perspective to recent research results, a short overview of the defining system parameters of RBC and the most common output quantities will be given. 
In thermal convection, the global flow structures and heat and momentum transport are determined mainly by the following system parameters: the Rayleigh number, $R a$, the Prandtl number, $P r$, and the aspect ratio of the container, $\Gamma$. Those are defined as

$$
R a \equiv \alpha g \Delta H^{3} /(\kappa \nu), \quad \operatorname{Pr} \equiv \nu / \kappa, \quad \Gamma \equiv D / H,
$$

respectively (Zwirner et al., 2020a).

Here $\alpha$ denotes the isobaric thermal expansion coefficient, $\nu$ the kinematic viscosity, $\kappa$ the thermal diffusivity of the fluid, $g$ the acceleration due to gravity, $\Delta \equiv T_{+}-T_{-}$the difference between the temperatures at the heated plate, $T_{+}$, and at the cooled plate, $T_{-}, H$ the distance between the plates and $D$ the diameter of the plates (for a cylindrical cell) (Zwirner et al., 2020a).

The main response characteristics of a natural convective system are the mean total heat flux across the heated/cooled plates, $q$, normalised by the conductive part of the total heat flux, $\hat{q}$, i.e. the Nusselt number, $N u$, and the Reynolds number, Re:

$$
N u \equiv q / \hat{q}, \quad R e \equiv L U / \nu .
$$

Here $U$ is the reference velocity, which is usually determined by either the maximum of the time-averaged velocity along the plates or by $\langle\mathbf{u} \cdot \mathbf{u}\rangle^{1 / 2}$, i.e. it is based on the mean kinetic energy; $\mathbf{u}$ is the velocity vector-field and $\langle\cdot\rangle$ denotes the average in time and over the whole convection cell (Zwirner et al., $2020 a)$.

Though these integral quantities are usually measured, major fascination lies also in the flow structures of thermal convection, starting from the diverse patterns that thermal convection develops close to onset (Bodenschatz et al., 2000) to the vorticity filaments of turbulent thermal convection. Over the years various experimental techniques — such as shadowgraphy, arrays of thermal probes, Doppler-velocimetry and particle image velocimetry (PIV) - have been utilized to visualize and quantify those flow structures. Furthermore, the advancement of computer technology facilitates highly-resolved simulations to solve the underlying equations and thus the detailed visualization and complete accessibility of the flow structures becomes possible.

Theory of turbulent thermal convection. One important research question is, how the volume-averaged heat and momentum transport depend on the input parameters, i.e. on $R a$ and $P r$. Starting from the marginal stability idea 
(a)

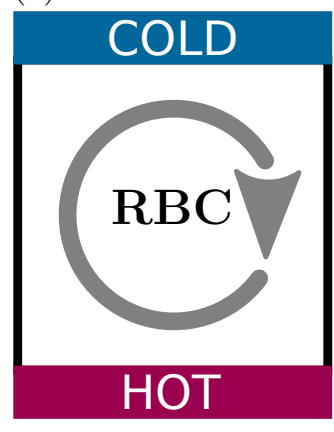

(b)

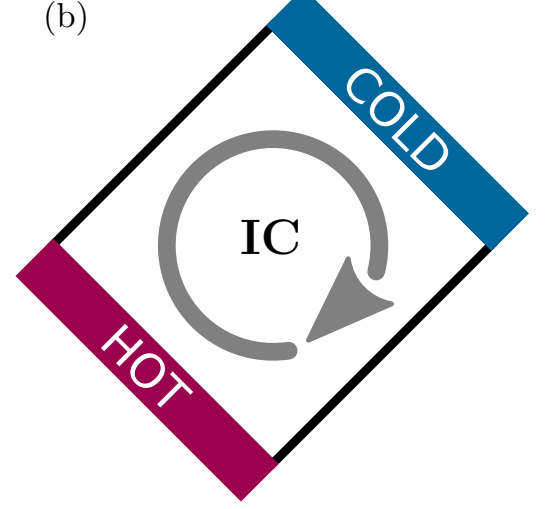

(c)

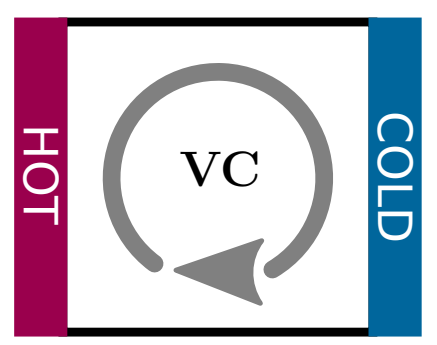

Figure 1.1: Sketch of the investigated configurations: (a) Rayleigh-Bénard convection, (b) inclined convection, (c) vertical convection.

by Malkus (1954), many attempts have been made to find a unifying theory that answers this question. Grossmann \& Lohse (2000, 2001, 2011) developed a scaling theory which is based on a decomposition into the boundary-layer and bulk contributions of the time- and volume-averaged kinetic $\left(\epsilon_{u}\right)$ and thermal $\left(\epsilon_{\theta}\right)$ dissipation rates, for which analytical relations with $N u, R a$ and $\operatorname{Pr}$ exist. Equating $\epsilon_{u}$ and $\epsilon_{\theta}$ to their estimated either bulk or BL contributions and employing in the BL dominated regimes the Prandtl-Blasius scaling (Prandtl, 1905; Blasius, 1908; Landau \& Lifshitz, 1987; Schlichting \& Gersten, 2000), theoretically possible limiting scaling regimes were derived and a smooth transition between the limiting regimes is provided by introducing crossover functions. The theory allows to predict $N u$ and $R e$ in RBC, while the pre-factors need to be fitted with the latest experimental and numerical data, see Stevens et al. (2013) and Shishkina et al. (2017) (Zwirner et al., 2020a).

Thermal convection in liquid metals. The main characteristic of liquid metals is, that $\operatorname{Pr} \ll 1$. This means, the thermal diffusion, $\kappa$, is much larger than the momentum diffusion, $\nu$, and therefore, thermal diffusion is much faster than momentum diffusion. This leads, among others things, to very thin viscous boundary layers at large $R a$. The effect of the Prandtl number on the flow structure can be seen by comparing figures $1.2(\mathrm{a})$ and (b), which depict the instantaneous temperature isosurfaces for a fluid of $\operatorname{Pr}=1$ and $\operatorname{Pr}=0.0094$, respectively. In figure 1.2(a) one can clearly see multiple thermal plumes that extend from the thermal boundary layers, as it is common for $\operatorname{Pr} \gtrsim 1$. Furthermore, the bulk region of the flow is well mixed at temperatures within less than $\pm 10 \%$ of the temperature difference $\Delta$. For low-Prandtl-number fluids, this structure changes and merely one large plume is observed, which extends verti- 



Figure 1.2: Visualization of the instantaneous flow of RBC in a cylinder $(\Gamma=1)$ from DNS data at (a, c) $R a=10^{9}, \operatorname{Pr}=1$ and (b, d) $R a=1.67 \times 10^{7}, \operatorname{Pr}=0.0094$. (a, b) Temperature isosurfaces $T / \Delta \in\{ \pm 0.5, \pm 0.4, \pm 0.3, \pm 0.2, \pm 0.1\}$ and (c, d) the isosurface where the local heat flux equals the time averaged heat flux $\left(\left(u_{z} T-\kappa \partial_{z} T\right) /(\kappa \Delta / H)=N u\right)$ coloured by temperature. Note that the cold (blue) part of the temperature scale is set to be almost completely transparent in order to gain a better view inside the flow. The cell is viewed from a slightly elevated position and black circles are drawn to emphasis the cylinder boundaries. 
cally across the whole cell. In the low- $\operatorname{Pr}$ case, the temperature isosurfaces are also much smoother, however, the velocity field is more turbulent, as one can see in figure 1.2(d), where the instantaneous heat flux is shown as an isosurface coloured by temperature. Note, the convective heat transport is basically the product of the vertical velocity and temperature. If one compares this visualization for different Prandtl number, cf. figures 1.2(c) and (d), one can immediately notice that the low-Prandtl-number visualization shows a slightly more vigorous isosurface of the heat flux. The Grashof number, $\mathrm{Gr} \equiv \mathrm{Ra} / \mathrm{Pr}$, for both compared cases is of the same order, however it is slightly larger for the low-Prandtl-number case and hence this flow is more vigorous.

Although the knowledge on thermal convection in liquid metals is required for the development of safe and efficient liquid metal heat exchangers, the experimental database of the corresponding measurements remains to be quite restricted, due to difficulties in conducting measurements in liquid metals. A few measurements of the scaling relations of $N u$ vs. $R a$ in liquid-metal Rayleigh-Bénard convection have been conducted, those are listed in table 1.1.

In nature, turbulent thermal convection at low Prandtl numbers is present, for example, in the surfaces of stars, where the Prandtl number varies from $10^{-8}$ to $10^{-4}$ (Spiegel, 1962; Hanasoge et al., 2016). It also takes place in the core of the earth, driving the geodynamo (Christensen \& Tilgner, 2004). Furthermore, turbulent thermal convection in liquid metals is relevant in engineering applications, especially in cooling systems of tokamaks and fast breeder reactors (Zhilin et al., 2009; Belyaev et al., 2013). Liquid sodium is of particular interest because of its very low Prandtl number $(\operatorname{Pr} \approx 0.009)$ and it is widely used as a cooling agent in fast neutron reactors (Heinzel et al., 2017) (Zwirner et al., 2020a).

Direct numerical simulations. Conducting high-fidelity direct numerical simulations (DNS) of natural thermal convection at high $R a$ and very low $\operatorname{Pr}$ is challenging. Very fine meshes in space and short steps in time are required due to the necessity to resolve the Kolmogorov microscales in the bulk of the flows as well as in the viscous BLs (Shishkina et al., 2010). Thus, there are only a few DNS of thermal convection for a combination of large $R a$ and very low Prandtl numbers $(\operatorname{Pr} \leq 0.025)$, which, moreover, have been conducted exclusively for the Rayleigh-Bénard configuration (Schumacher et al., 2016; Scheel \& Schumacher, 2016, 2017; Horn \& Schmid, 2017; Vogt et al., 2018) (Zwirner \& Shishkina, 2018).

Along with the advances in computer technology it is now possible to inves- 


\begin{tabular}{|c|c|c|c|c|c|}
\hline Reference & Fluid & $\operatorname{Pr}$ & & $R a$ & $\gamma$ \\
\hline McDonald \& Connolly (1960) & $\mathrm{Na}$ & 0.009 & $4.8 \times 10^{6}$ & $4 \times 10^{7}$ & $0.32 \pm 0.01$ \\
\hline Dropkin \& Somerscales (1965) & $\mathrm{Hg}$ & 0.020 & $10^{6}$ & $2 \times 10^{7}$ & $1 / 3^{\dagger}$ \\
\hline Rossby (1969) & $\mathrm{Hg}$ & 0.025 & $2 \times 10^{4}$ & $5 \times 10^{5}$ & $0.26 \pm 0.01$ \\
\hline Takeshita et al. (1996) & $\mathrm{Hg}$ & 0.024 & $1.5 \times 10^{6}$ & $7.6 \times 10^{7}$ & $0.27 \pm 0.02$ \\
\hline Cioni et al. (1997) & $\mathrm{Hg}$ & 0.025 & $7 \times 10^{6}$ & $4.5 \times 10^{8}$ & $0.26 \pm 0.02$ \\
\hline Cioni et al. (1997) & $\mathrm{Hg}$ & 0.025 & $4.5 \times 10^{8}$ & $2.1 \times 10^{9}$ & $0.20 \pm 0.02$ \\
\hline Glazier et al. (1999) & $\mathrm{Hg}$ & 0.024 & $2 \times 10^{5}$ & $7 \times 10^{10}$ & $0.29 \pm 0.01$ \\
\hline Horanyi et al. (1999) & $\mathrm{Na}$ & 0.006 & $2 \times 10^{4}$ & $5 \times 10^{6}$ & $0.25 \pm 0.01$ \\
\hline King \& Aurnou (2013) & $\mathrm{Ga}$ & 0.025 & $2 \times 10^{6}$ & $10^{8}$ & $0.25 \pm 0.01$ \\
\hline Scheel \& Schumacher (2017) & $\mathrm{Hg}^{*}$ & 0.021 & $3 \times 10^{5}$ & $4 \times 10^{8}$ & $0.26 \pm 0.01$ \\
\hline Scheel \& Schumacher (2017) & $\mathrm{Na}^{*}$ & 0.005 & $3 \times 10^{5}$ & $10^{7}$ & $0.27 \pm 0.01$ \\
\hline Zürner et al. (2019) & GaInSn & 0.029 & $4 \times 10^{6}$ & $6 \times 10^{7}$ & $0.27 \pm 0.02$ \\
\hline Zwirner et al. $(2020 a)$ & $\mathrm{Na}$ & 0.009 & $5.3 \times 10^{6}$ & $2.4 \times 10^{7}$ & $0.22 \pm 0.06$ \\
\hline
\end{tabular}

Table 1.1: Overview of the scaling exponents of $N u \sim R a^{\gamma}$. Direct Numerical Simulations are marked with *. Note, in Zwirner et al. (2020a) the scaling exponents were measured experimentally, however, numerical results are presented as well, for a fixed Rayleigh number (cf. chapter 4).

tigate thermal convection in low-Prandtl-number fluids by means of DNS. This work adds more data points to this sparsely investigated parameter space.

Turbulent superstructures. The turbulent thermal convective flow is composed of several interacting flow structures, for example the large-scale circulation, boundary layers and larger, so-called superstructures.

Stevens et al. (2018) investigated superstructures in turbulent thermal convection at Rayleigh numbers up to $R a=10^{9}$, Prandtl number, $\operatorname{Pr}=1$, and very large aspect ratios of up to $\Gamma=L / H=128$, where $L$ is the lateral extent of the square hot and cold plates. Their numerical results demonstrate the existence of superstructures with a horizontal extent of six to seven times of the cell height, hence, these structures are much larger than the LSC present in the aspect ratio one. Furthermore, they found that the peak position of the temperature variance and turbulent kinetic energy spectra converges for aspect ratios of approximately $\Gamma=64$.

Pandey et al. (2018) studied numerically the temporal evolution of similar superstructures in aspect ratio $\Gamma=L / H=25$ for a huge range of Prandtl numbers $(0.005 \leq \operatorname{Pr} \leq 70)$ and Rayleigh numbers up to $R a=10^{7}$, and revealed a separation of large-scale, slowly evolving structures and small-scale, rapidly changing structures. Their results suggest a correlation of the thermal plume 
ridges in the BLs and the characteristic scales of the superstructures. To reveal this separation, they averaged the flow over timescales that are larger than the free-fall timescale and smaller than the horizontal dissipation timescale.

Vogt et al. (2018) found in laboratory experiments and corresponding DNS that in liquid gallium and cylindrical convection cells of a greater-than-unity aspect ratio (up to $\Gamma=D / H=2$ ), the LSC takes the form of a "jumping rope vortex".

Krug et al. (2020) discovered that the temperature and velocity superstructures in turbulent RBC are highly correlated and coherent, which was not observed in previous studies using an ordinary averaging strategy (i.e. Stevens et al. (2018)). This was found by a scale-by-scale analysis of the linear coherence spectrum using DNS data. Additionally, results by Krug et al. (2020) suggest, that the superstructure scale increases with $R a$ and it is independent of the vertical position inside the cell.

Green et al. (2020) applied a filtering approach to their DNS data to study the kinetic energy and temperature variance budgets. They found that the energy transfer rate between the large-scale flow structures and small-scale fluctuations has a height-dependent, complex structure (i.e. boundary layer and bulk features).

However, not only large aspect ratios give rise to superstructures, also lateral confinement, as discussed in the next paragraph, creates large flow structures. Those are built out of several rolls stacked on top of each other.

Laterally confined convection. Apart from Prandtl number and Rayleigh number, the geometry of the container plays an important role for the global flow structure formation. In several experimental and numerical studies, the effect of lateral geometric confinement in rectangular containers (width-to-height aspect ratio $\Gamma \leq 1 / 3$ ) was studied (Wagner \& Shishkina, 2013; Huang et al., 2013; Chong et al., 2015; Chong \& Xia, 2016; Chong et al., 2018). Experiments by Huang et al. (2013) revealed a confinement induced increase in convective heat transport in water $\left(\operatorname{Pr}=4.38,8.6 \times 10^{7} \leq R a \leq 2.5 \times 10^{9}\right)$. They observed more coherent plumes, also inside the bulk, and these plumes suppress the LSC. In a numerical study, Wagner \& Shishkina (2013) found that at fixed Prandtl number $\left(\operatorname{Pr}=0.786,10^{5} \leq R a \leq 10^{9}\right)$, the heat and mass transport is reduced and the global flow structure changes with increasing confinement. Chong et al. (2015) found that for a fixed Prandtl number $(\operatorname{Pr}=4.38$, $10^{7} \leq R a \leq 10^{10}$ ) there is an optimal aspect ratio at which the heat transport is maximized and it decreases with increasing Rayleigh number. Recently, 
Chong et al. (2018) found that the Prandtl number determines whether the optimal aspect ratio exists or not. Thus, the Prandtl number plays an important role in the formation of the global flow structure and heat transport in confined containers. For $\operatorname{Pr} \geq 0.5\left(R a=10^{8}\right)$, an enhanced heat transport was observed, where the optimal aspect ratio decreases with increasing Prandtl number, but for $\operatorname{Pr} \leq 0.5$ a gradual reduction of the heat transport with confinement was observed, as it was reported by Wagner \& Shishkina (2013) (Zwirner \& Shishkina, 2018). The single-roll LSC is known to be more efficient in the heat transport than its double-roll form, as it was proved in the measurements (Xi \& Xia, 2008; Weiss \& Ahlers, 2013) (Zwirner et al., 2020a). The global flow structure under geometrical confinement changes in a way that clusters of thermal plumes develop near the lateral walls and change the plume covered area of the BLs, which appears to be associated with an increased heat transport. For all Prandtl numbers, the confinement induced friction causes a decrease of the Reynolds number (Zwirner \& Shishkina, 2018). The present work considers lateral confinement as well. In chapter 2 , the focus lies on a thermal convection inside a small-aspect-ratio cylinder, where the cell height, $H$, is five times larger than the diameter, $D$. In chapter 3 the occurrence of multiple rolls on top of each other inside a slender cell is investigated and their formation will be linked to the elliptical instability.

Inclined convection. The concept of inclined convection (IC) is a modification of RBC, where the fluid layer between the parallel plates is tilted with respect to the direction of gravity, and both buoyancy and shear act on the flow (cf. figure 1.1b) (Zwirner et al., 2020a). In the literature, the inclined convection studies can be classified into two groups: in the first, small tilt angles are considered to study the effect of imperfectly aligned RBC cells, or to fix the plane of the large-scale circulation in order to measure its properties. In the second, also larger inclination angles up to $\beta=\pi / 2$ are considered. In the present work, this full range of inclination angles is explored.

Small tilt angles were investigated in several studies. Sun et al. (2005) observed a decrease in Nusselt number of approximately $2 \%$ for a tilt of $2^{\circ}$ in their diameter-to-height aspect ratio $1 / 2$ cylindrical cell filled with water $\left(\operatorname{Pr}=5.3, R a=5.3 \times 10^{10}\right)$. Weiss \& Ahlers (2013) conducted experiments in a similar cell filled with water $\left(\operatorname{Pr}=4.38, R a \simeq 10^{10}\right)$ for tilt angles $\beta<7^{\circ}$ and, in contrast to Sun et al. (2005), found a very small increase in the Nusselt number of approximately $0.3 \%$. In $\operatorname{RBC}(\beta=0)$ they observed transitions between two different flow states: a single-roll state and a double-roll state. 
They proposed that the double-roll state produces a lower global heat flux and this state is generally suppressed by inclination, that is, for larger inclination angle the system spends less time in the double-roll state (Zwirner \& Shishkina, 2018). There are further studies investigating the dependence of a small tilt angle on the heat transport and those find only a small effect, see Ciliberto et al. (1996); Cioni et al. (1997); Chillà et al. (2004); Ahlers et al. (2006b); Roche et al. (2010); Wei \& Xia (2013).

Recent investigations of inclined thermal convection at inclination angles of up to $90^{\circ}$ revealed a complex non-monotonic behaviour of the heat and momentum transport. One example is the work by Shishkina \& Horn (2016), where inclined convection was investigated in a cylindrical cell for Rayleigh numbers from $10^{6}$ to $10^{8}$ and Prandtl numbers from 0.1 to 100 . Guo et al. (2015) observed experimentally in a rectangular cell a monotonic decrease in heat transport for $\operatorname{Pr} \simeq 6.3$ and $R a \simeq 4.42 \times 10^{9}$, while the Reynolds number showed a non-monotonic behaviour. Dropkin \& Somerscales (1965) did experiments of inclined convection for various Rayleigh numbers and Prandtl numbers inside a large aspect ratio cell and found that the scaling relation of the Nusselt number is similar for all inclination angles, only the prefactors differ (Zwirner \& Shishkina, 2018).

Inclined convection in liquid metals is especially fascinating. Previous studies showed that the heat transport at any inclination angle $\left(0^{\circ}<\beta \leq 90^{\circ}\right)$ is larger than that in the RBC case, see Frick et al. (2015), Mamykin et al. (2015), Vasil'ev et al. (2015b), Kolesnichenko et al. (2015), and Khalilov et al. (2018). These experiments showed that the convective heat transport is most efficient neither for $\beta=0^{\circ}$ nor for $\beta=90^{\circ}$, but for an intermediate inclination angle. Furthermore, this maximum of heat transport at an intermediate inclination angle becomes more pronounced if the aspect ratio is smaller, i.e. the cylinder becomes very long and slender. Vasil'ev et al. (2015a) observed an approximately tenfold increase in the heat transport for an inclination angle of $\beta \approx 65^{\circ}$ at Rayleigh number $R a_{D}=2.4 \times 10^{6}$, based on the cell diameter. In their experiments, liquid sodium and a cylinder of the diameter-to-height aspect ratio $\Gamma=1 / 20$ were used. This effect of a huge increase in the Nusselt number might be exploited in technical applications, e.g. in cooling systems for nuclear power plants or computers (Zwirner \& Shishkina, 2018).

So far, all available experimental and numerical results on IC show that the $N u(\beta) / N u(0)$ dependence is a complex function of $R a, \operatorname{Pr}$ and $\Gamma$, which cannot be represented as a simple combination of their power functions (Zwirner et al., 2020a). Therefore, this work aims to shed light on this dependence. 
Vertical convection. At an inclination of $\beta=\pi / 2$, the second limiting case of inclined convection is reached. This case is usually called vertical convection (VC) since the heated and cooled plates are vertical compared to the original $\mathrm{RBC}$ configuration. In the literature, it is also called convection in cavities or side-heated convection.

Shear plays a key role in VC, as the heated and cooled plates are parallel to gravity, see $\mathrm{Ng}$ et al. $(2015,2017)$ and Shishkina (2016). The theoretical scaling relations of the Nusselt and Reynolds number with respect to $R a$ and $\operatorname{Pr}$ are already known for laminar flow, as derived by Shishkina (2016), however, for the turbulent case there is no model so far. While the general ansatz of the Grossmann-Lohse theory can be applied, there is a closure problem in VC, which makes it necessary to model the buoyancy flux parallel to the plates and it is not easy to find a proper model (Zwirner \& Shishkina, 2018).

Note that even for the same geometry, $R a$ and $P r$, not only the heat transport in $\mathrm{VC}$ differs from that in $\mathrm{RBC}$, but also the flow states can be different. For example, in a cylindrical container of $\Gamma=1$, for $\operatorname{Pr}=1$ and $R a=10^{8}$, the VC flow is steady, while the RBC flow is turbulent (Shishkina \& Horn, 2016).

Further motivation to investigate VC is that until now the dependences of $N u$ and $R e$ on $R a$ and $P r$ in VC have been investigated less than those in RBC. They were measured experimentally by Belmonte et al. (1995), who found a scaling exponent close to $2 / 7$ for Rayleigh numbers of $4 \times 10^{5}<R a \leq 10^{11}$ and Prandtl number Pr $=0.7$. Previous experimental and numerical studies of free thermal convection under an imposed horizontal temperature gradient (i.e., VC) reported the scaling exponent $\gamma$ in the power law $N u \sim R a^{\gamma}$, varying from $1 / 4$ to $1 / 3$. In laminar VC, it is about $1 / 4$ (Schmidt \& Beckmann, 1930; Lorenz, 1934; Saunders, 1939; Churchill \& Chu, 1975), being slightly larger for very small $R a$, where the geometrical cell confinement influences the heat transport (Versteegh \& Nieuwstadt, 1999; Yu et al., 2007; Kis \& Herwig, 2012; $\mathrm{Ng}$ et al., 2015). The scaling exponent $\gamma$ is also larger for very high $R a$, where, with growing $R a$, the VC flows become first transitional (Ng et al., 2017) and later on fully turbulent (George \& Capp, 1979; Fujii et al., 1970). Note that all the mentioned experiments and simulations of $\mathrm{VC}$ were conducted for fluids of $\operatorname{Pr}$ about or larger than 1 (Zwirner \& Shishkina, 2018).

For the case of small $P r$, in the experiments by Frick et al. (2015) and Mamykin et al. (2015) on turbulent VC in liquid sodium $(\operatorname{Pr} \approx 0.01)$ in elongated cylinders, significantly larger scaling exponents were observed, due to the geometrical confinement. Thus, for a cylinder with $H=5 D$ and the Rayleigh numbers, based on the cylinder diameter, up to $10^{7}$, Frick et al. (2015) obtained 
$N u \sim R a^{0.43}$ and $R e \sim G r^{0.44}$, where $G r$ is the Grashof number (Zwirner \& Shishkina, 2018).

A final remark on $\mathrm{VC}$ of small-Prandtl-number fluids: usually, the absolute heat transport is larger, compared to RBC, although the flow is less turbulent.

Outline. The present study is dedicated to investigate the interplay of confinement and inclination of the convection cell, in the case of low Prandtl numbers $(\operatorname{Pr} \leq 1)$. The heat and momentum transport, as well as the emerging flow structures are studied. In chapter 2 the focus is set on inclined convection under confinement of the cell. Afterwards, the appearance of multi-roll flow modes inside a slender cylinder is discussed for the RBC case (chapter 3). Eventually, the influence of the flow structure dynamics on the heat transport is discussed in chapter 4 , for the very low-Prandtl number fluid, liquid sodium, and aspect ratio one. This work concludes with a discussion and summary of the obtained results. 



\section{Confined inclined thermal convection in low-Prandtl-number fluids}

This chapter is a reprint of the following publication:

Lukas Zwirner \& Olga Shishkina: Confined inclined thermal convection in low-Prandtl-number fluids. J. Fluid Mech. 850, 984-1008, (2018).

Reproduced with permission.

Own contribution: conduction of all direct numerical simulations, writing the code to analyse and visualize the data, analysis of all the data, writing the first draft of the paper.

Abstract. Any tilt of a Rayleigh-Bénard convection cell against gravity changes the global flow structure inside the cell, which leads to a change of the heat and momentum transport. Especially sensitive to the inclination angle is the heat transport in low-Prandtl-number fluids and confined geometries. The purpose of the present work is to investigate the global flow structure and its influence on the global heat transport in inclined convection in a cylindrical container of diameter-to-height aspect ratio $\Gamma=1 / 5$. The study is based on direct numerical simulations where two different Prandtl numbers $\operatorname{Pr}=0.1$ and 1.0 are considered, while the Rayleigh number, $R a$, ranges from $10^{6}$ to $10^{9}$. For each combination of $R a$ and $\operatorname{Pr}$ the inclination angle is varied between 0 and $\pi / 2$. An optimal inclination angle of the convection cell, which provides the maximal global heat transport, is determined. For inclined convection we observe the formation of two system-sized plume columns, a hot and a cold one, that impinge on the opposite boundary layers. These are related to a strong increase in the heat transport.

\subsection{Introduction}

Thermal convection is omnipresent in nature and technical applications. The model system of Rayleigh-Bénard convection (RBC), where a fluid is confined between a hot bottom plate and a cold top plate, has been thoroughly studied for over a century (Bodenschatz et al., 2000; Ahlers et al., 2009; Roche et al., 2010; Chillà \& Schumacher, 2012). In most of the studies, moderate and relatively large diameter-to-height aspect ratios $\Gamma$ of the convection cell $(\Gamma \geq 1 / 2)$ and fluids of relatively large Prandtl number $\operatorname{Pr} \geq 0.7$ were considered.

Along with the advances in computer technology, it is now possible to in- 
vestigate thermal convection in low-Prandtl-number fluids by means of direct numerical simulations (DNS). Studies of convection in fluids like liquid mercury or gallium $\left(\operatorname{Pr}=0.021, R a \leq 4 \times 10^{8}\right)$ in a cylindrical container of diameter-to-height aspect ratio $\Gamma=1$ (Schumacher et al., 2015, 2016; Scheel \& Schumacher, 2016) revealed several differences to convection in high-Prandtlnumber fluids. In general, convection in these fluids is characterized by the production of coarse thermal plumes and the development of more vigorous turbulent flows. The production of only a few but coarse plumes is caused by the increased thermal diffusivity at low Prandtl numbers and is accompanied by a smaller maximum in the root-mean-square temperature profile. Especially for their lowest Prandtl number $(\operatorname{Pr}=0.005)$ Scheel \& Schumacher (2016) observed only one hot and one cold large plume rather than multiple ones. Furthermore, in low-Prandtl-number RBC, the viscous boundary layer (BL) is embedded in the thermal BL. With decreasing Prandtl number the viscous BL becomes thinner, while the thermal one becomes thicker and the profiles of the root-mean-square velocities are enhanced, which indicates a more vigorous flow. Scheel \& Schumacher (2016) examined the local viscous $\mathrm{BL}$ thickness and the local shear Reynolds number $R e_{s h}$ (based on the viscous BL thickness and the root-mean-square velocity at the BL edge) and found that the boundary layer is in a transient state with multiple turbulent patches when $R e_{s h} \geq 250$. These turbulent patches cover a larger fraction $(\sim 5 \%)$ of the BL at smaller Prandtl numbers and/or higher Rayleigh numbers. Schumacher et al. (2015) found that the Kolmogorov-like cascade is extended at both the large- and small-scale ends for smaller Prandtl numbers at constant Grashof number $G r=R a / P r=4.76 \times 10^{8}$. Additionally, the enstrophy generation is enhanced because of the higher Reynolds numbers achieved by higher Rayleigh/lower Prandtl numbers.

Apart from $\operatorname{Pr}$ and the Rayleigh number $R a$, the geometry of the container plays an important role. In several experimental and numerical studies, the effect of lateral geometric confinement in rectangular containers (width-to-height aspect ratio $\Gamma \leq 1 / 3$ ) was studied (Wagner \& Shishkina, 2013; Huang et al., 2013; Chong et al., 2015; Chong \& Xia, 2016; Chong et al., 2018). Experiments by Huang et al. (2013) revealed a confinement-induced increase in convective heat transport in water $\left(\operatorname{Pr}=4.38,8.6 \times 10^{7} \leq R a \leq 2.5 \times 10^{9}\right)$. They observed more coherent plumes, also inside the bulk, and these plumes suppress the large-scale circulation (LSC). In a numerical study, Wagner \& Shishkina (2013) found that at fixed Prandtl number $\left(\operatorname{Pr}=0.786,10^{5} \leq R a \leq 10^{9}\right)$ the heat and mass transport is reduced and the global flow structure changes with 
increasing confinement. Chong et al. (2015) found that for a fixed Prandtl number $\left(\operatorname{Pr}=4.38,10^{7} \leq R a \leq 10^{10}\right)$ there exists an optimal aspect ratio at which the heat transport is maximized and it decreases with increasing Rayleigh number. Recently, Chong et al. (2018) found that the Prandtl number determines whether the optimal aspect ratio exists or not. Thus, the Prandtl number plays an important role in the formation of the global flow structure and heat transport in confined containers. For $\operatorname{Pr} \geq 0.5\left(R a=10^{8}\right)$ an enhanced heat transport was observed, where the optimal aspect ratio decreases with increasing Prandtl number, but for $\operatorname{Pr} \leq 0.5$ a gradual reduction of the heat transport with confinement was observed, as reported by Wagner \& Shishkina (2013). The global flow structure under geometrical confinement changes in such a way that clusters of thermal plumes develop near the lateral walls and change the plume-covered area of the boundary layers, which appears to be associated with an increased heat transport. For all Prandtl numbers, the confinement-induced friction causes a decrease of the Reynolds number.

Previous investigations of thermal convection in inclined cylinders with $\Gamma=1$ showed a complex non-monotonic behaviour of the Nusselt number and the Reynolds number (Shishkina \& Horn, 2016). In these investigations the classical Rayleigh-Bénard cell was tilted up to $\pi / 2$, i.e. up to the case of so-called vertical convection (VC), while the Rayleigh number ranged from $10^{6}$ to $10^{8}$ and the Prandtl number ranged from 0.1 to 100. Guo et al. (2015) observed, in their experiments in a rectangular cell (length-to-height aspect ratio 1 and width-to-height aspect ratio 0.25), a monotonic decrease in heat transport with an inclination angle of up to $\pi / 2$, for $P r \simeq 6.3$ and $R a \simeq 4.42 \times 10^{9}$. The Reynolds number behaves differently with inclination: first it increases until a certain inclination angle and then monotonically decreases. Sun et al. (2005) observed a decrease in Nusselt number of approximately $2 \%$ for a tilt of $2^{\circ}$ in their diameter-to-height aspect ratio $1 / 2$ cylindrical cell filled with water $\left(\operatorname{Pr}=5.3, R a=5.3 \times 10^{10}\right)$. Weiss \& Ahlers (2013) conducted experiments in a similar cell filled with water $\left(\operatorname{Pr}=4.38, R a \simeq 10^{10}\right)$ for tilt angles $\beta<7^{\circ}$ and, in contrast to Sun et al. (2005), found a very small increase in the Nusselt number of approximately $0.3 \%$. In $\operatorname{RBC}(\beta=0)$ they observed transitions between two different flow states: a single-roll state and a double-roll state. They proposed that the double-roll state produces a lower global heat flux and this state is generally suppressed by inclination, that is, for larger inclination angle the system spends less time in the double-roll state. In this article we will also show the existence of these states and confirm their influence on the 
global heat transport. Dropkin \& Somerscales (1965) did experiments on inclined convection for various Rayleigh numbers and Prandtl numbers inside a large-aspect-ratio cell. They found that the scaling relation of the Nusselt number is similar for all inclination angles, only the prefactor differs.

For vertical convection $(\beta=\pi / 2)$, the second limiting case of our study, the scaling relations of the Nusselt and Reynolds numbers with $\operatorname{Ra}$ and $\operatorname{Pr}$ are already known for the laminar case (Shishkina, 2016). In an attempt to adapt the unifying theory for RBC (Grossmann \& Lohse, 2000; Stevens et al., 2013) to turbulent vertical convection, $\mathrm{Ng}$ et al. (2015) showed that the principal ideas can be applied to VC, but for closure it is necessary to model the buoyancy flux parallel to the plates. The closure problem exists also in the general case of inclined convection, where the buoyancy flux splits into two components, as we will see in section 2.2.2. Vertical convection was also observed experimentally, for example by Belmonte et al. (1995), who investigated the heat transport and temperature profiles in a box.

The present work aims to study the effect of combining the three aforementioned aspects: low Prandtl number, lateral geometric confinement and inclination of the convection cell. Recent experiments of this kind, as conducted by Frick et al. (2015), showed a doubled global heat transport for the inclined case compared to that in RBC. In their experiments, a cylindrical cell $(\Gamma=1 / 5)$ with liquid sodium $\left(\operatorname{Pr} \approx 0.008, R a \approx 10^{9}\right)$ was used. Teimurazov \& Frick (2017) compared the results obtained in these experiments and in largeeddy simulations (LES). Their simulations indicated that the LSC plays an important role in the heat transport of inclined convection. Furthermore they compared two different boundary conditions (BC): constant temperature and constant heat transport at the plates. The latter boundary condition is closer to the experimental reality, because of the small difference in thermal conductivity of the plate and the fluid. The constant-heat-transport BC leads to a Nusselt number, that is approximately $15-20 \%$ higher than the Nusselt number obtained for the constant-temperature BC. Proceeding to even stronger confinement, Vasil'ev et al. (2015a) observed an approximately tenfold increase in the heat transport for an inclination angle of $\simeq 65^{\circ}$ at Rayleigh number $R a_{D}=2.4 \times 10^{6}$, based on the cell diameter. In their experiments, liquid sodium and a cylinder of diameter-to-height aspect ratio $\Gamma=1 / 20$ was used. This effect of a huge increase in the Nusselt number might be exploited and can be very important for numerous technical applications, e.g. in cooling systems for nuclear power plants or computers; therefore it is necessary to gain further insight into its mechanism. However, especially in experiments with 
liquid metals, it is impossible to access the flow structure in detail. Therefore, we aim to close this gap of knowledge with our direct numerical simulations.

In the present study, we will show that inclination and confinement of the convection cell influence the global flow structure in convection of low-Prandtlnumber fluids, which manifests in the development of two plume columns inside the inclined cell, a hot one and a cold one. The formation and the strength of these plume columns are the main features that determine the enhancement of the heat transport.

\subsection{Analytical relations and numerical simulations}

\subsubsection{Governing equations}

Inclined thermal convection in Boussinesq approximation is described by the following Navier-Stokes and energy equations:

$$
\begin{aligned}
\partial_{t} \mathbf{u}+\mathbf{u} \cdot \nabla \mathbf{u} & =-\nabla p+\nu \nabla^{2} \mathbf{u}+\alpha\left(T-T_{0}\right) g \mathbf{e}, \\
\partial_{t} T+\mathbf{u} \cdot \nabla T & =\kappa \nabla^{2} T
\end{aligned}
$$

with $T_{0}=\left(T_{+}+T_{-}\right) / 2, \mathbf{u}=\left(u_{r}, u_{\phi}, u_{z}\right)$ and $\mathbf{e}=(-\sin \beta \cos \phi, \sin \beta \sin \phi, \cos \beta)$, where $\beta$ is the angle of inclination, $\Delta=T_{+}-T_{-}$the temperature difference between the hot and cold plates, $g$ the gravitational acceleration, $\kappa$ the thermal diffusivity, $\nu$ the kinematic viscosity and $\alpha$ the thermal expansion coefficient. The inclination angle $\beta$ is varied between $\beta=0$, which corresponds to $\mathrm{RBC}$ and $\beta=\pi / 2$, so-called vertical convection (VC) (Ng et al., 2015, 2017; Shishkina, 2016). Additionally, the incompressibility condition, $\nabla \cdot \mathbf{u}=0$, is fulfilled and the following boundary conditions are applied: no-slip velocity at all walls, constant temperature at the top/bottom plates and adiabatic sidewalls. Non-dimensionalization of these equations results in three governing input parameters: the Rayleigh number $R a$, Prandtl number $\operatorname{Pr}$ and aspect ratio $\Gamma$ :

$$
R a \equiv \frac{\alpha g \Delta H^{3}}{\kappa \nu}, \quad \operatorname{Pr} \equiv \frac{\nu}{\kappa}, \quad \Gamma \equiv \frac{D}{H},
$$

where $D$ and $H$ are the diameter and height of the cylinder.

In the present work we consider two Prandtl numbers, $\operatorname{Pr}=0.1$ and 1 , and the Rayleigh numbers $R a=10^{6}, 10^{7}, 10^{8}$ and $10^{9}$. For each combination of the Rayleigh number and Prandtl number, we conduct simulations with 11 different inclination angles distributed equally from 0 to $\pi / 2$. The aspect ratio is fixed to $\Gamma=1 / 5$, except for $\operatorname{Pr}=1$ and $R a=10^{9}$, where we present results also for the aspect ratio $\Gamma=1$. 
The main output quantities are the global heat transport represented by the dimensionless Nusselt number

$$
N u \equiv \frac{\left\langle T u_{z}\right\rangle_{\mathrm{A}}-\kappa\left\langle\partial_{z} T\right\rangle_{\mathrm{A}}}{\kappa \Delta / H}
$$

and the Reynolds number based on the kinetic energy,

$$
R e \equiv \frac{H \sqrt{\left\langle\mathbf{u}^{2}\right\rangle}}{\nu}
$$

Here $\langle\cdot\rangle_{\mathrm{A}}$ denotes the averaging in time and over a cross-section at any distance from the heated plate and $\langle\cdot\rangle$ denotes the averaging in time and over the entire volume.

We define the velocity across the plates, the so-called wind, as

$$
U_{\mathrm{w}} \equiv \max _{z}\left(\sqrt{\left\langle u_{r}^{2}\right\rangle_{\mathrm{A}}+\left\langle u_{\phi}^{2}\right\rangle_{\mathrm{A}}}\right)
$$

and the Reynolds number $R e_{\mathrm{w}}$ based on the wind as

$$
R e_{\mathrm{w}} \equiv \frac{H U_{\mathrm{w}}}{\nu}
$$

Following Teimurazov \& Frick (2017), we define the Reynolds number based on the volume-averaged velocity fluctuations, or small-scale Reynolds number, as

$$
R e_{u^{\prime}} \equiv \frac{H \sqrt{\left\langle\left(\mathbf{u}-\langle\mathbf{u}\rangle_{\mathrm{t}}\right)^{2}\right\rangle}}{\nu}
$$

where $\langle\cdot\rangle_{\mathrm{t}}$ denotes the averaging in time and the large-scale Reynolds number

$$
R e_{U} \equiv \frac{H \sqrt{\left\langle\langle\mathbf{u}\rangle_{\mathrm{t}}^{2}\right\rangle_{\mathrm{V}}}}{\nu}
$$

Note that it is different from (2.5) because $\langle\mathbf{u}\rangle_{\mathrm{t}}^{2} \neq\left\langle\mathbf{u}^{2}\right\rangle_{\mathrm{t}}$.

\subsubsection{Exact relations for the dissipation rates in inclined convection}

The Nusselt number in RBC is related to the kinetic and thermal energy dissipation rates,

$$
\left\langle\varepsilon_{u}\right\rangle \equiv \nu\left\langle\left[\partial_{i} u_{j}\right]^{2}\right\rangle \quad \text { and } \quad\left\langle\varepsilon_{\theta}\right\rangle \equiv \kappa\left\langle\left[\partial_{i} \theta\right]^{2}\right\rangle,
$$






Figure 2.1: Sketch of an inclined convection setup. $D$ is the diameter, $H$ is the height of the cylinder, $\beta$ the inclination angle, $T_{+}\left(T_{-}\right)$the temperature of the hot (cold) plate and $g$ is the acceleration due to gravity.

respectively, as follows (Shraiman \& Siggia, 1990):

$$
\begin{aligned}
\left\langle\varepsilon_{u}\right\rangle & =\frac{\nu^{3}}{H^{4}} \frac{R a}{\operatorname{Pr}^{2}}(N u-1) \text { and } \\
\left\langle\varepsilon_{\theta}\right\rangle & =\frac{\kappa \Delta^{2}}{H^{2}} N u .
\end{aligned}
$$

These fundamental relations are widely used, for example to validate the computational mesh in numerical simulations (see section 2.2.3). They are also the starting point of the Grossmann \& Lohse (2000) theory (GLT) for the scaling relations of the mean heat and momentum transport in different regimes of RBC. However, in inclined convection, the relation (2.11) generally does not hold, but it is possible to get a similar relation for $\left\langle\varepsilon_{u}\right\rangle$. In the inclined case, this relation has an additional term, which describes the heat transport parallel to the plates and which can be modelled. $\mathrm{Ng}$ et al. (2015) pointed out that, in order to extend the GLT to vertical convection, it is necessary to find a closure model for this term.

Multiplying scalarly the Navier-Stokes equation (2.1) with the velocity vector field $\mathbf{u}$, averaging in time and space and utilizing the definition of the Nusselt number (2.4), we get

$$
\frac{\nu^{3}}{H^{4}} \frac{R a}{P r^{2}}(N u-1)=\frac{\left\langle\varepsilon_{u}\right\rangle+\alpha g\left\langle T u_{\|}\right\rangle \sin \beta}{\cos \beta},
$$

where $\left\langle T u_{\|}\right\rangle$denotes the time- and volume-averaged heat transport parallel to the plates in the $x$ direction (see figure 2.1). For the two limiting cases of RBC 
$(\beta=0)$ and $\operatorname{VC}(\beta=\pi / 2)$ we get

$$
\begin{aligned}
\left\langle\varepsilon_{u}\right\rangle_{\beta=0} & =\alpha g\left\langle T u_{z}\right\rangle \text { and } \\
\left\langle\varepsilon_{u}\right\rangle_{\beta=\pi / 2} & =-\alpha g\left\langle T u_{\|}\right\rangle
\end{aligned}
$$

respectively. From the equation (2.13) and the definition of the Nusselt number (2.4) we obtain

$$
\frac{H^{4}}{\nu^{3}} \frac{\operatorname{Pr}^{2}}{R a} \frac{\left\langle\varepsilon_{u}\right\rangle}{N u-1}=\cos \beta-\frac{\left\langle T u_{\|}\right\rangle}{\left\langle T u_{z}\right\rangle} \sin \beta
$$

This equation shows how the ratio of $\left\langle\varepsilon_{u}\right\rangle$ and the Nusselt number depends on the inclination angle. Note that $\left\langle T u_{\|}\right\rangle /\left\langle T u_{z}\right\rangle$ depends on the inclination angle as well.

\subsubsection{Direct numerical simulations and mesh resolution}

To investigate inclined thermal convection, three-dimensional DNS were performed in a cylindrical domain. The momentum and energy equations (2.1) and (2.2) are solved by the GoldFish code (Shishkina et al., 2015; Shishkina \& Wagner, 2016; Shishkina \& Horn, 2016; Kooij et al., 2018). The utilized mesh needs to resolve the smallest scales of the flow like the Batchelor and Kolmogorov scales (Shishkina et al., 2010). Since here we consider the case of $\operatorname{Pr} \leq 1$, the Kolmogorov scale $\eta=\left(\nu^{3} / \varepsilon_{u}\right)^{1 / 4}$ is the most demanding scale in our DNS. As a finite volume scheme is used in the simulations, the local characteristic mesh size is defined as $h=\left(r \Delta_{r} \Delta_{\phi} \Delta_{z}\right)^{1 / 3}$, where $\Delta_{r}, \Delta_{\phi}$ and $\Delta_{z}$ are the local mesh spacings in the $r, \phi$ and $z$ directions, respectively. For a good mesh resolution $h$ should be less than or at least similar to $\eta$. This ratio $h / \eta$ is a local quantity, since a non-equidistant mesh is used and the kinetic energy dissipation rate varies locally as well. To get a single characteristic number to describe the mesh resolution, the maximum of the average in the $\phi$ direction is used:

$$
h_{\eta}=\max _{r, z}\langle h / \eta\rangle_{\mathrm{t}, \phi}
$$

Furthermore, deviations from the exact relations (2.12) and (2.13) can serve as reliable indicators for the accuracy of the numerical simulations. Therefore, we monitor also the quantities

$$
\mathcal{E}_{\mathrm{u}} \equiv \max _{\beta \in[0, \pi / 2)}\left\{\frac{H^{4}}{\nu^{3}} \frac{\operatorname{Pr}^{2}}{R a} \frac{\left\langle\varepsilon_{u}\right\rangle+\alpha g\left\langle T u_{\|}\right\rangle \sin \beta}{(N u-1) \cos \beta}-1\right\} \text {, }
$$

(for $\beta<\pi / 2$ only, since the limit $\beta \rightarrow \pi / 2$ is difficult to obtain numerically) 
and

$$
\mathcal{E}_{\theta} \equiv \max _{\beta \in[0, \pi / 2]}\left\{\frac{H^{2}}{\kappa \Delta^{2}} \frac{\left\langle\varepsilon_{\theta}\right\rangle}{N u}-1\right\} .
$$

In table 2.1 these values as well as the number of mesh points are listed for all performed simulations. Additionally, for $R a=10^{7}, \operatorname{Pr}=1, \beta / \pi=0$ and $\Gamma=1 / 5$ a simulation was run also on a finer mesh, similar to the one for $R a=10^{8}$. It showed good agreement with the lower resolved mesh: averaged quantities such as the Nusselt number and Reynolds number deviate only approximately $1 \%$.

\subsection{Results}

\subsubsection{The effect of inclination}

We consider the global transport quantities in the case of inclined convection. Inclination of the cell, in the investigated parameter range, usually leads to an increase in the global heat transport until a certain optimal inclination angle $\beta^{*}$ where the heat transport is maximal. Further inclination leads to a decrease of the global heat transport (see figure 2.2a,c,e). For the Reynolds number, $R e$, based on the kinetic energy (2.5), the behaviour with inclination is similar to that for the heat transport. However, the maximum of the Reynolds number, based on kinetic energy, is usually observed for angles smaller than or equal to $\beta^{*}$ and the relative decrease of $R e$ is much stronger near the VC case (see figures $2.2 \mathrm{~b}, \mathrm{~d}, \mathrm{f})$. Also the relative increase of the Reynolds number is usually less than the relative increase of the Nusselt number (cf. figure 2.2c,e with $2.2 \mathrm{~d}, \mathrm{f})$. The only exception from the above observations is the case of $\operatorname{Pr}=1$ and $R a=10^{7}$, where inclination always leads to a decrease of the heat and momentum transport. The reason for this will be discussed further below.

The inclination angle $\beta^{*}$ for the optimal heat transport is plotted against the Rayleigh number in figure 2.3. The figure shows that $\beta^{*}$ depends nonmonotonically on $R a$. However, one can see that, at a lower Prandtl number and/or larger aspect ratio, the optimal heat transport is reached at a larger inclination angle.

A closer look at the instantaneous flow fields (see figure 2.4 for $\operatorname{Pr}=1$ and figure 2.5 for $\operatorname{Pr}=0.1$ ) reveals some expected and also some new features, which can be associated with the inclination. Inclining the cylinder forces the direction of the LSC to be fixed, which results in a very structured flow. This is illustrated by those flow fields in figures 2.4 and 2.5, where the streamlines align nicely in the case of inclined convection.

At the lowest Rayleigh number, $R a=10^{6}$, the spatial temperature dis- 






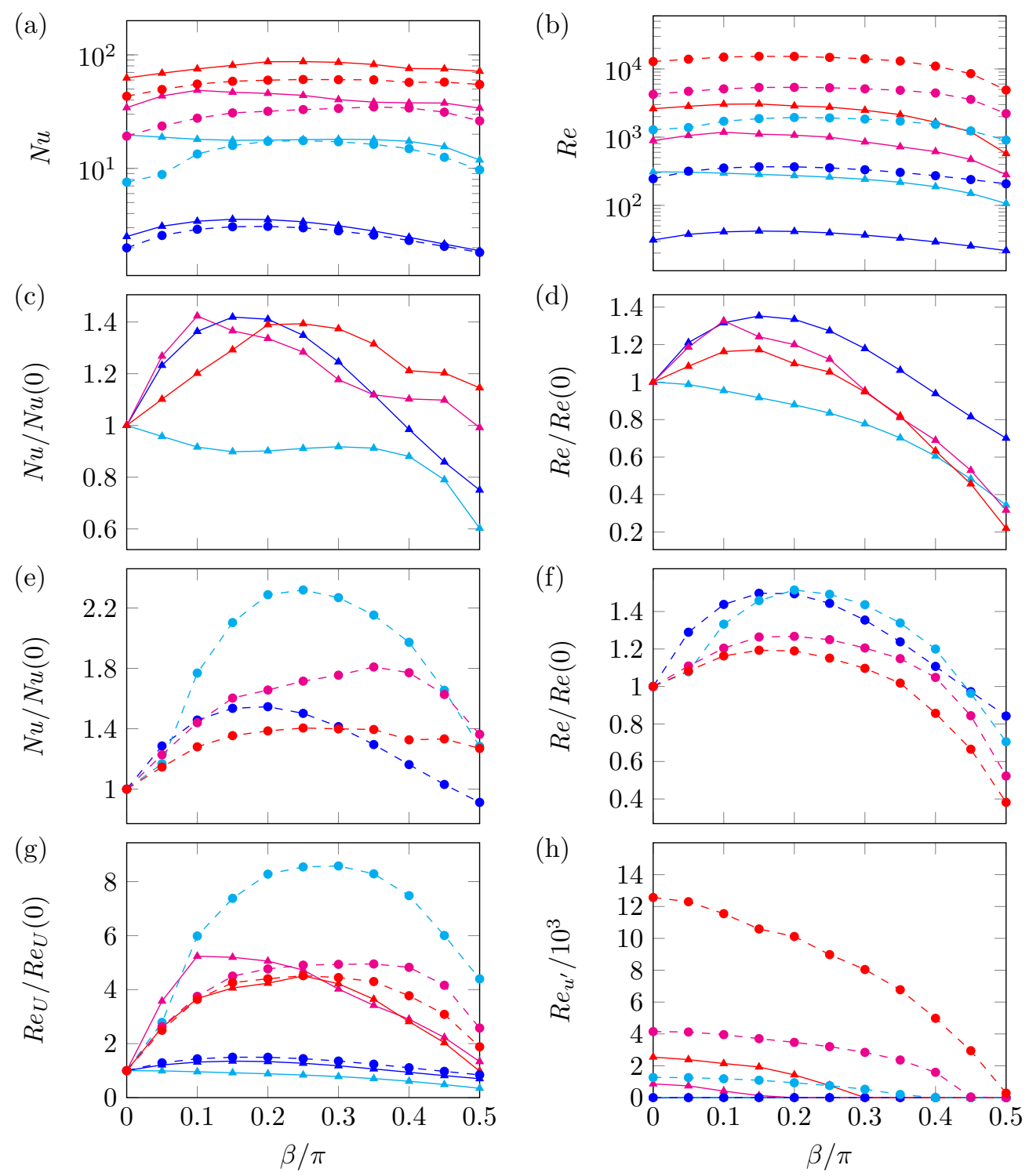

Figure 2.2: The absolute and normalized (with respect to RBC) Nusselt and Reynolds numbers plotted against the inclination angle $\beta$ for $\operatorname{Pr}=1$ (solid lines) and $\operatorname{Pr}=0.1$ (dashed lines). Rayleigh numbers are $10^{6}$ (blue), $10^{7}$ (cyan), $10^{8}$ (magenta) and $10^{9}$ (red). (a) Absolute Nusselt number. (b) Absolute Reynolds number, based on kinetic energy. (c) Normalized Nusselt number for $\operatorname{Pr}=1$. (d) Normalized Reynolds number for $\operatorname{Pr}=1$. (e) Normalized Nusselt number for $\operatorname{Pr}=0.1$. (f) Normalized Reynolds number for $\operatorname{Pr}=0.1$. (g) Normalized large-scale Reynolds number. (h) Absolute small-scale Reynolds number. 


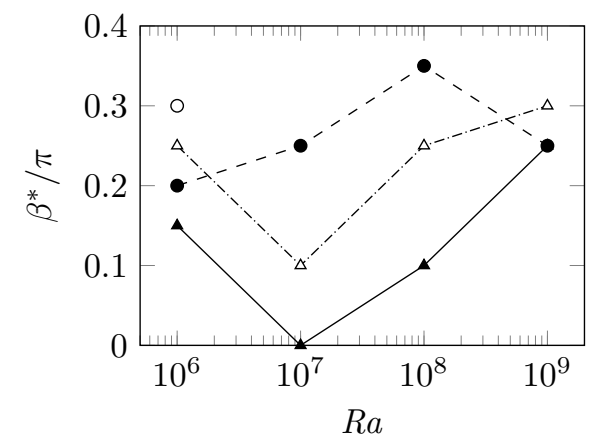

Figure 2.3: Inclination angle $\beta^{*}$ for the optimal heat transport, as a function of $R a$, for $\operatorname{Pr}=1$ (triangles) and $\operatorname{Pr}=0.1$ (circles), for the aspect ratios $\Gamma=1 / 5$ (filled symbols) and $\Gamma=1$ (open symbols). The data for $\Gamma=1, R a<10^{9}$ are adopted from Shishkina \& Horn (2016).

tribution is similar to that of the conductive case and there are no single plumes visible for either of the investigated Prandtl numbers. These flow states are steady solutions of the governing equations (2.1) and (2.2) because the Reynolds number based on the velocity fluctuations, $R e_{u^{\prime}}$, vanishes. Additionally, the streamlines are aligned and show a flow field with a smooth laminar-like LSC (see figures 2.4a and 2.5a).

While increasing the Rayleigh number and thus the thermal driving $\left(\operatorname{Ra}=10^{7}, \operatorname{Pr}=1\right)$, we still observe a laminar-like flow with an LSC but the spatial temperature distribution looks completely different now (figure 2.4b). The temperature at the rising/falling side of the LSC is close to the temperature of the respective BL. This picture is reminiscent of hot/cold channels or plume columns which span the whole cylinder and is observed for all inclination angles. Nevertheless, in this case there are no distinguishable plumes apart from the rising/falling ones and the flow is also laminar-like. As we will see, these plume columns appear to play an important role in the increase of the heat transport in inclined convection (cf. section 2.3.2).

For $R a=10^{7}$ and $\operatorname{Pr}=1$, the RBC flow provides the optimal heat transport. This can be explained by the fact that the plume columns are already present in the flow configuration without inclination $(\beta / \pi=0)$, see figure $2.4 \mathrm{~b}$, while the strength of the LSC decreases with increasing inclination (cf. figure $2.2 \mathrm{c}, \mathrm{g}$ ).

A further increase of the Rayleigh number to $R a=10^{8}$ leads to a development of a quite vigorous flow in RBC (see figure $2.4 \mathrm{c}, \beta=0$ ) and in this case the rising and falling plumes appear chaotically, leading to a complicated flow structure (see supplementary movie $1^{*}$ ). Also, the small-scale Reynolds number $\left(R e_{u^{\prime}}=865\right)$ is not vanishing any more, which means that there are

*https://doi.org/10.1017/jfm.2018.477 
(a)



(c)

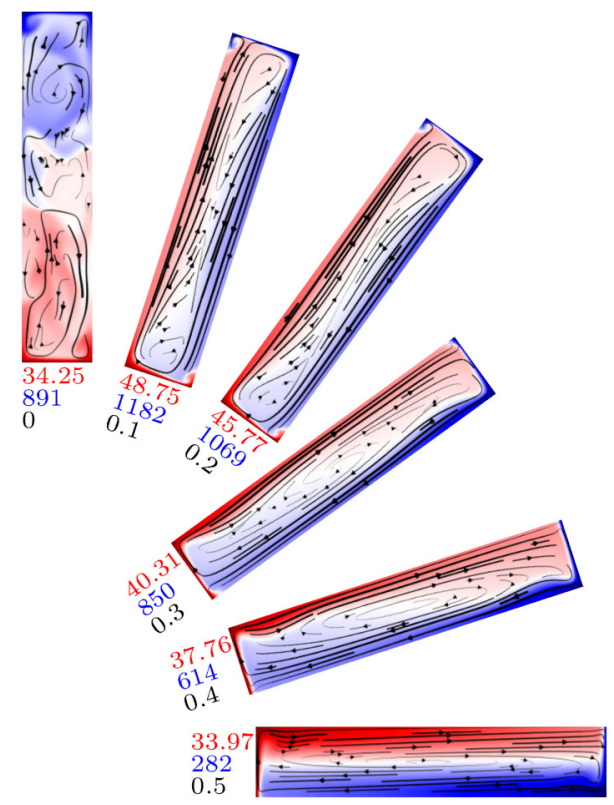

(b)

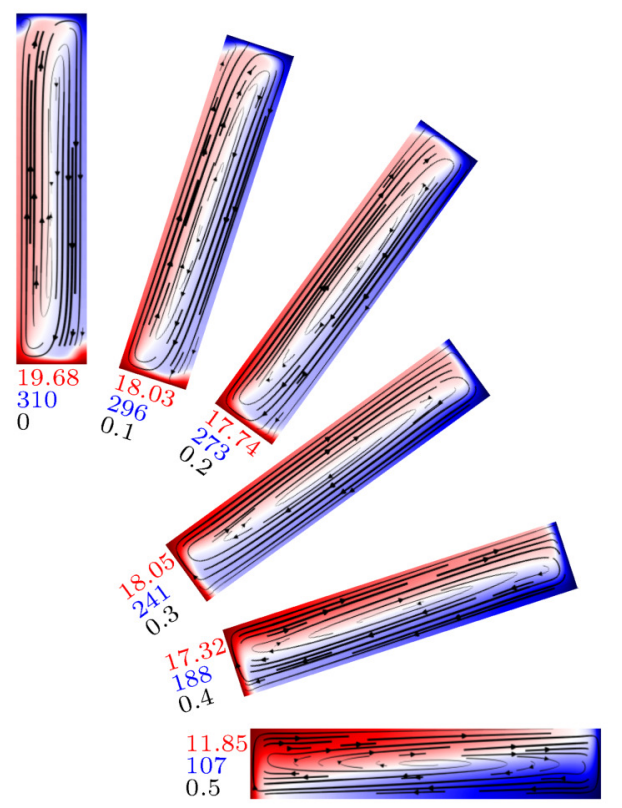

(d)



Figure 2.4: Vertical cross-sections through the instantaneous flow fields for $\operatorname{Pr}=1$ and (a) $R a=10^{6}$, (b) $R a=10^{7}$, (c) $R a=10^{8}$ and (d) $R a=10^{9}$. The colour scale is from hot (red) to cold (blue). The coloured numbers are the Nusselt number (red), Reynolds number (blue) and inclination angle $\beta / \pi$ (black). The thickness of the black streamlines reflects the normalized velocity magnitude. 
(a)

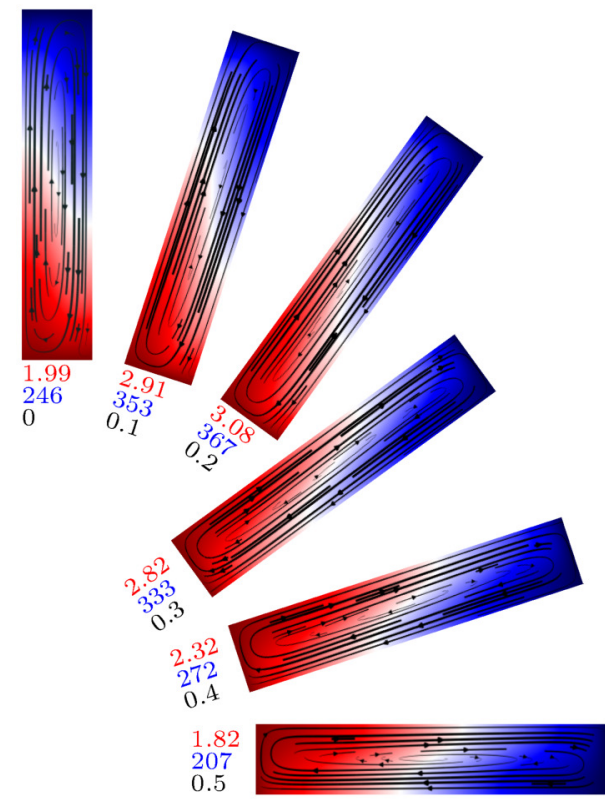

(c)

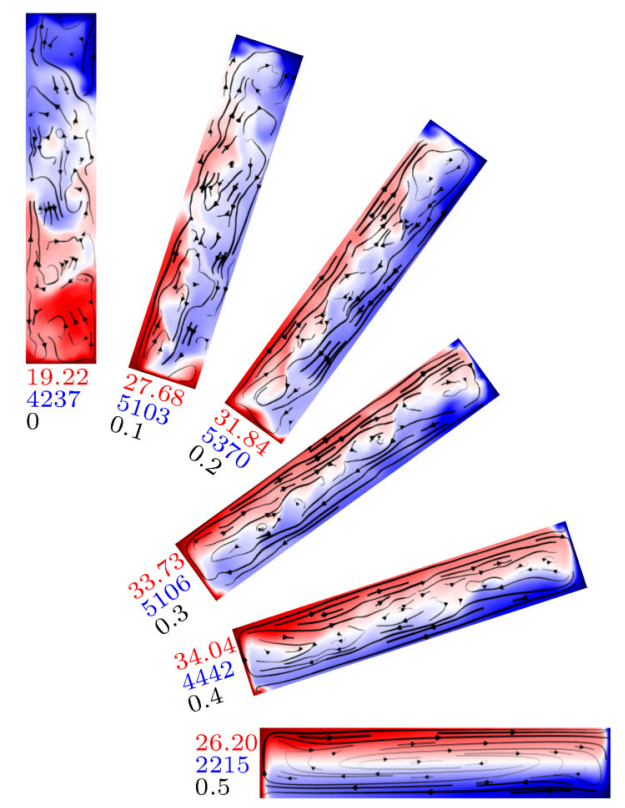

(b)



(d)

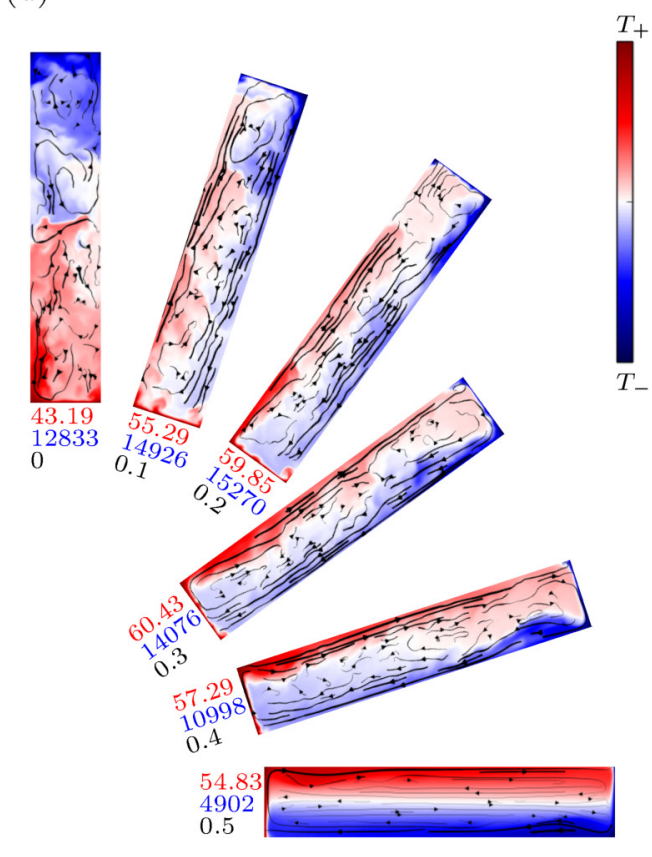

Figure 2.5: Vertical cross-sections through the instantaneous flow fields for $\operatorname{Pr}=0.1$ and (a) $R a=10^{6}$, (b) $R a=10^{7}$, (c) $R a=10^{8}$ and (d) $R a=10^{9}$. The coloured numbers are the Nusselt number (red), Reynolds number (blue) and inclination angle $\beta / \pi$ (black). Colour scale and streamlines as in figure 2.4. 


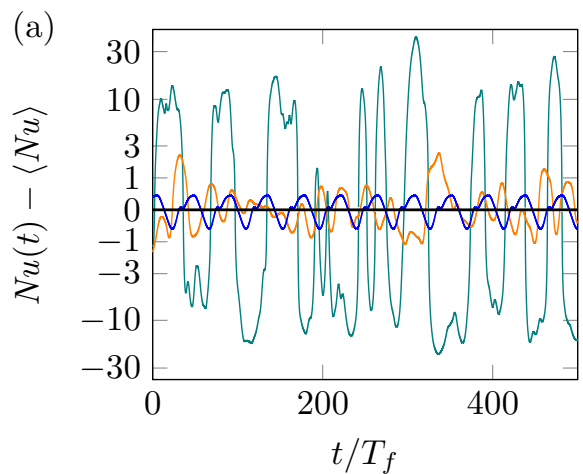

(c)

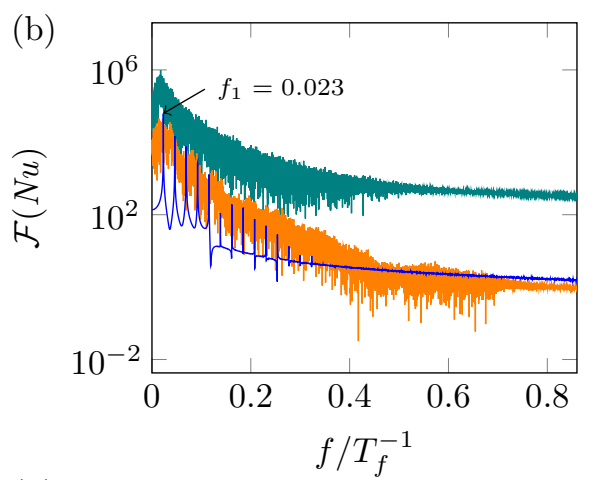

(d)
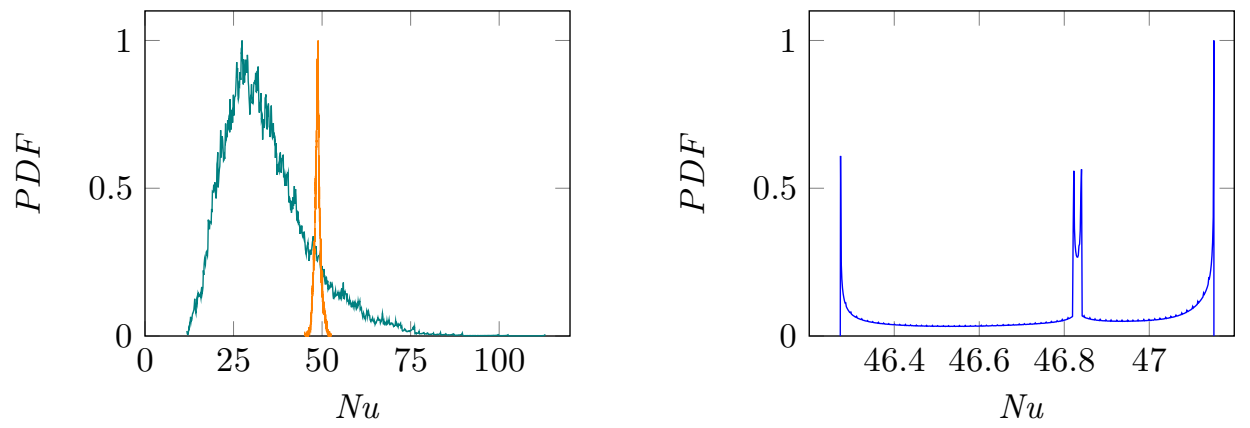

Figure 2.6: The temporal behaviour of the Nusselt number for $R a=10^{8}$, $\operatorname{Pr}=1, \Gamma=1 / 5$ and $\beta / \pi=0$ (green), $\beta / \pi=0.1$ (orange) and $\beta / \pi=0.15$ (blue). The time scale is based on $T_{f}=\sqrt{R /(\alpha g \Delta)}$. (a) Deviations of the Nusselt number from its mean value (note that the positive and negative scale is logarithmic) versus time. (b) Amplitude of the Fourier spectrum $\mathcal{F}$ of the Nusselt number. (c, d) Probability density functions (PDF) of the Nusselt number normalized by the respective maximum. Here the instantaneous Nusselt number $N u(t)$ is determined by averaging over the entire volume. 
significant fluctuations in the velocity field. Inclining the cell leads to a decrease in the small-scale Reynolds number (figure $2.2 \mathrm{~h}$, solid magenta line) and for an inclination angle of $\beta \approx 0.2 \pi$, the flow becomes steady. The emergence of the plume columns is observed at $\beta=0.1 \pi$, where also the maximal Nusselt number is obtained (cf. figures 2.4b and 2.4c). Furthermore, the largescale Reynolds number reaches its maximum there, too, while the small-scale Reynolds number decreases by approximately $50 \%$ compared to that in RBC.

Except for the case of highest Rayleigh number, $R a=10^{9}$, and lowest Prandtl number, $\operatorname{Pr}=0.1$, we always observe a transition to a steady flow field when the inclination angle is increased. Hence, we will discuss this transition to steady flow using this parameter set of $R a=10^{8}$ and $\operatorname{Pr}=1$ as an example, since it demonstrates all kinds of behaviour, from a turbulent flow in RBC to the formation of plume columns, as well as an oscillatory state and finally, for a sufficiently large inclination angle, a steady flow. Therefore, we pick the five most interesting inclination angles, namely $\beta / \pi=0$ (RBC), $\beta^{*} / \pi=0.1$ (optimal heat transport), $\beta / \pi=0.15$ (oscillatory state), $\beta / \pi \approx 0.2$ (steady flow) and $\beta=\pi / 2$ (VC) to discuss the behaviour. The time evolution of the Nusselt number is displayed in figure 2.6a and for a better understanding we also provide the amplitude of the Fourier spectrum (figure 2.6b) and the probability density function (p.d.f.) of the Nusselt number (figure 2.6c,d).

In $\mathrm{RBC}$ we observe strong fluctuations of the Nusselt number, where the standard deviation is approximately $35 \%$ of the mean value and the p.d.f. shows a wide tail at higher Nusselt numbers. In the case of optimal heat transport $(\beta=0.1 \pi)$, the probability density function of the Nusselt number is narrow, almost symmetric and the standard deviation is only approximately $1.8 \%$ of the mean value. The width of the p.d.f. of the Nusselt number is decreasing with inclination, which is due to the strong stratification of the flow. The third case, as already mentioned, is special, because we observe an oscillatory state that leads to a periodic variation of the Nusselt number. The main frequency is $f_{1}=0.023 T_{f}^{-1}$ and the Fourier spectrum (figure 2.6b) also shows multiples of this main frequency. Apparently this frequency is also characteristic (strongest) for the less inclined cases, which show a continuous spectrum. Below, the oscillating case will be further discussed. At $\beta / \pi \approx 0.2$, the flow becomes steady and the Nusselt number does not change in time any more, so it is of no interest to look at the p.d.f. or spectrum for any larger inclination angles. In $\mathrm{VC}$ we observe a temperature distribution which is strongly stratified with respect to the direction of gravity. For large $R a$ and $\operatorname{Pr}=0.1$, the Nusselt numbers in $\mathrm{VC}$ are larger than those in RBC 
(cf. figure 2.2e).

Supplementary movie $2^{\dagger}$ shows the temperature isosurfaces of the oscillating flow field $(\beta / \pi=0.15)$. There is a torsional oscillation visible in the flow, which is asymmetric in azimuthal direction. Oscillating states were also observed for $\mathrm{RBC}$ in an aspect-ratio-one cell between the onset of convection and fully turbulent flow, by Verzicco \& Camussi (1997). This suggests that the route to turbulence in inclined convection is similar to RBC.

The qualitative picture of the flow structure, i.e. the emergence of plume columns, at the optimal inclination angle $\beta^{*}$ (with respect to the Nusselt number) appears to be similar for both investigated Prandtl numbers and $R a \geq 10^{7}$. This means that the maximal heat transport is reached as soon as the plume columns are fully developed, i.e. they span the whole cylinder, and the largescale Reynolds number is also very close to its maximal value. Thus, our simulations evince similar behaviour of the small- and large-scale Reynolds number as observed in the LES by Teimurazov \& Frick (2017). They claimed that these observations are an indicator that the LSC is playing an important role in the heat transport in inclined convection.

As expected, for a lower Prandtl number $(P r=0.1)$ turbulent flows are obtained already at lower Rayleigh numbers and they remain turbulent until larger inclination angles. This is underlined quantitatively by the small-scale Reynolds numbers, which are plotted in figure 2.2(h). One can see from these data that the transition from a turbulent to a steady flow happens at larger inclination angles for lower Prandtl numbers or higher Rayleigh numbers.

Although the Prandtl number in the experiments by Frick et al. (2015) and LES by Teimurazov \& Frick (2017) is significantly lower than $\operatorname{Pr}=0.1$, there are certain similarities between our and their results. We obtained a similar relative increase of the heat transport $\left(N u\left(\beta^{*}\right) / N u(0)\right)$. The studies showed also that, for otherwise similar set of parameters, a higher Nusselt number is obtained in VC rather than in RBC. In both the LES and our DNS, similar temperature profiles were obtained that demonstrated a non-vanishing gradient of the mean temperature in the bulk in RBC and vanishing gradient in inclined and vertical convection.

The highest relative increase in heat transport is observed for Rayleigh number $R a=10^{7}$ and Prandtl number $\operatorname{Pr}=0.1$. We see a vigorous flow, $R e_{u^{\prime}}=756$, and system-sized plume column formation that finally leads to an approximately 2.3 times stronger heat transport in the inclined case $\left(\beta^{*} \approx 0.25 \pi\right)$ compared to that in $\mathrm{RBC}$.

\footnotetext{
${ }^{\dagger}$ https://doi.org/10.1017/jfm.2018.477
} 


\subsubsection{Plume columns and the flow structure of enhanced heat transfer}

Since the plume columns appear to increase the heat transport as soon as they span the whole cylinder and, therefore, impinge on the opposite boundary layer, one might also compare the situation in inclined convection with that of impinging jets on a surface. When a jet impinges on a hot surface, the heat transport depends on many parameters, like the Reynolds number, Prandtl number,ratio of plate-distance to nozzle-diameter, and radial distance from the stagnation point (Jambunathan et al., 1992). It is known that the heat transport is generally larger in an impinging jet rather than in a parallel flow, but the heat transport decreases with the distance from the stagnation point of the jet. This was shown by various experiments like those by Huang \& ElGenk (1994). Under the assumption that the fully developed plume columns, spanning the whole cylinder, act like a jet impinging on a surface, it is reasonable to assume that this might cause the strongly increased heat transport. Additionally, such impinging zones are clearly seen in figure $2.7(\mathrm{~h}, \mathrm{k})$. From these panels it is apparent that the ratio of the impinging area to the plate area is much smaller in aspect ratio $\Gamma=1$ compared to $\Gamma=1 / 5$ and the heat transport behaves similarly, i.e. it is higher inside the slender cylinder. For a thorough analysis of the aspect ratios, see section 2.3.4.

Our slender geometry also shows some similarities of heat transport through an inclined vertical channel. Riedinger et al. (2013) carried out such experiments with a square channel of aspect ratio $1 / 4$, where the ends of the channel are connected to a hot/cold reservoir of water. They found similar behaviour in the inclined channel, like stratification due to gravity, and an apparently higher scaling exponent in the transition region to the turbulent regime (as discussed below in section 2.3.5). Furthermore, they also observed two nonmixing symmetrical jets with inclination angle $\beta / \pi>0$.

In general, stratification of the temperature field, which is induced by the cell inclination, supports the formation of the plume columns. On the other hand, high Rayleigh numbers enhance the turbulent mixing and thus break apart the plume columns. All this leads to the conclusion that, for larger $R a$, the optimal heat transfer is to be expected at larger values of $\beta / \pi$. A careful interpretation of figure 2.3 supports this trend. Here, one has to keep in mind that the plume columns exist only for $R a \geq 10^{7}$.

It is necessary to further discuss the physical processes that support the formation of plume columns, since the reported findings indicate the importance of the emerging plume columns to the increase in heat transport. Therefore, let us consider a hot thermal plume or some small patch of hot fluid that has just 
detached from the thermal BL and what will happen to it afterwards. There are two relevant time scales: the time that the plume needs to travel to the opposite BL, and the thermal diffusion time, during which the plume loses its thermal energy to the surrounding fluid. First, the thermal plume is collected by the LSC and therefore accelerates to the velocity of the LSC, $U=\sqrt{\left\langle\langle\mathbf{u}\rangle_{\mathrm{t}}^{2}\right\rangle_{\mathrm{V}}}$ (as used in (2.9)), which gives the travelling time scale, $\tau_{u}=H / U$. The relevant length scale for the travelling time is the height of the cylinder $H$, whereas we set the radius $R$ as the relevant length scale for the thermal diffusion time, $\tau_{\kappa}=R^{2} / \kappa$, since this is the approximate lateral extent the plume can gain, before it will interact with the cold plume channel. The lateral viscous diffusion time, $R^{2} / \nu$, is longer and hence not important in these considerations for $\operatorname{Pr} \leq 1$. Plume columns can be formed if the travelling time is less than or approximately the same as the thermal diffusion time, $H / U \lesssim R^{2} / \kappa$. From this we can estimate a threshold for our large-scale Reynolds number that is necessary to produce plume columns: $4 \Gamma^{2} \operatorname{Re} e_{U} \operatorname{Pr} \gtrsim 1$. As already discussed for $R a=10^{6}$ and both Prandtl numbers, we have a profile that is close to the conductive temperature distribution (no plume columns) and indeed we find $4 \Gamma^{2} R e_{U} \operatorname{Pr}<1$. For $R a=10^{7}$ and $\operatorname{Pr}=1$ we observe plume columns and $4 \Gamma^{2} \operatorname{Re} e_{U} \operatorname{Pr} \approx 3$. A further condition for the plume columns to exist is that the velocity fluctuations must not prevail over the mean flow.

\subsubsection{Single-roll and double-roll states in Rayleigh-Bénard convection}

For the RBC case of this parameter set, we find two possible states: a singleroll state and a double-roll state (figure 2.8). The single-roll state is used as the reference case for the relative change of the Nusselt number and Reynolds number, because in inclined convection the single-roll state dominates. As known, e.g. from experiments by Xi \& Xia (2008); Weiss \& Ahlers (2013), the heat flux in the double-roll state is smaller compared to that in a single-roll state. Our simulations confirm this fact, and we find a strong reduction, i.e. the heat transport in the double-roll state is only approximately $80 \%$ of the heat transport in the single-roll state for $R a=10^{6}$ and $\operatorname{Pr}=0.1$ (figures 2.8b-e). There is one further case where we observe a single-roll state and a double-roll state, which is $\operatorname{Ra}=10^{7}$ and $\operatorname{Pr}=1$ (figures $2.4 \mathrm{~b}$ and $2.8 \mathrm{f}, \mathrm{g}$ ).

Both states are steady (vanishing velocity fluctuations) but their occurrence depends on the initial flow fields of the simulation. This means that, unlike the experiment by Weiss \& Ahlers (2013), transitions between these states are not to be expected.

For a slightly higher Rayleigh number, $R a=2 \times 10^{7}$, we tried to find both 

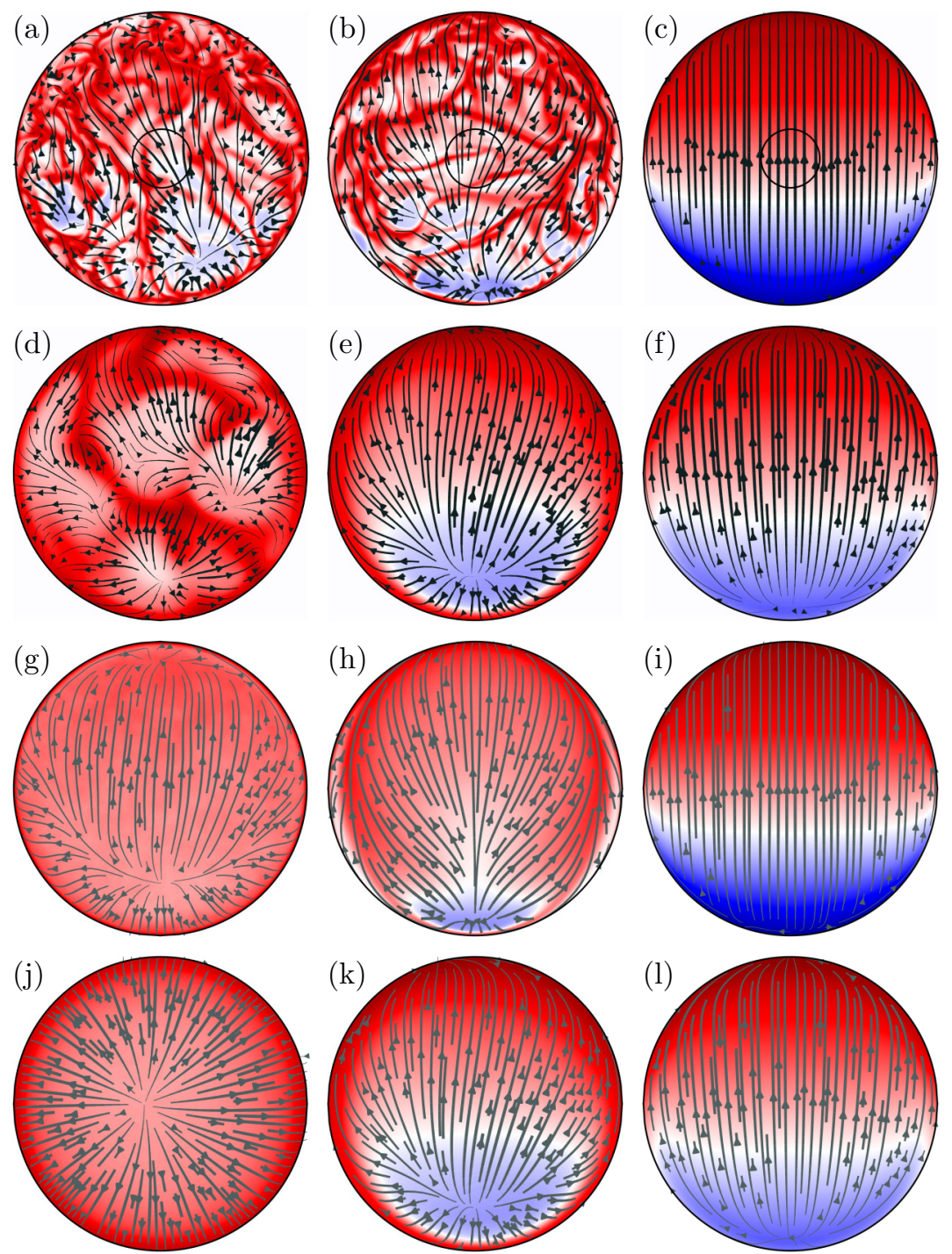

Figure 2.7: Cross-sections parallel to the heated plate at the edge of the thermal BL $\left(z=\delta_{\mathrm{T}}=H /(2 N u)\right)$ for $R a=10^{9}$ and $\operatorname{Pr}=1$ of (a-f) the instantaneous and (g-l) the time-averaged temperature and velocity fields (colour scale and streamlines as in figure 2.4). The aspect ratios are $(\mathrm{a}-\mathrm{c}, \mathrm{g}-\mathrm{i}) \Gamma=1$ and $(\mathrm{d}-\mathrm{f}, \mathrm{j}-\mathrm{l}) \Gamma=1 / 5$. All cross-sections are taken for three different inclination angles $\beta / \pi=0$ (first column), $\beta / \pi=0.25$ (second column) and $\beta / \pi=0.5$ (third column). The inner circles in (a-c) show the area of the cross-section in the case of $\Gamma=1 / 5$ for the same height as in (d-f), respectively. 
(a)

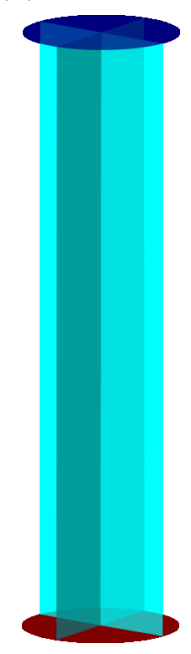

(b)

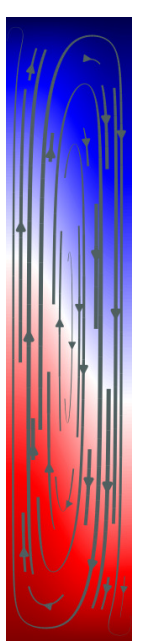

(c)

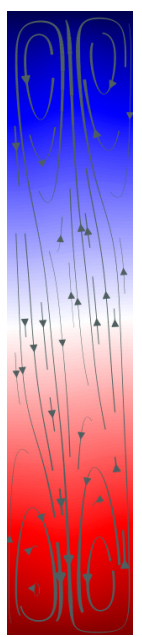

(d)



(e)

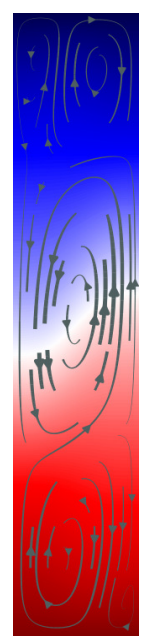

(f)

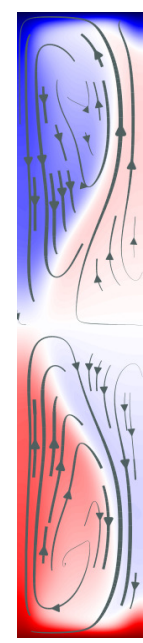

(g)

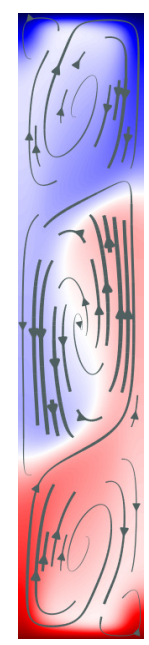

Figure 2.8: Vertical cross-sections sketched in (a) through the instantaneous flow fields of (b, c) the single-roll state for $R a=10^{6}$ and $\operatorname{Pr}=0.1$; (d, e) the double-roll state for $R a=10^{6}$ and $\operatorname{Pr}=0.1$; (f, g) the double-roll state for $R a=10^{7}$ and $\operatorname{Pr}=1$. Vertical cross-sections through (b, d, f) the plane of the rolls and (c, e, g) the plane perpendicular to it. Colour scale and streamlines as in figure 2.4.
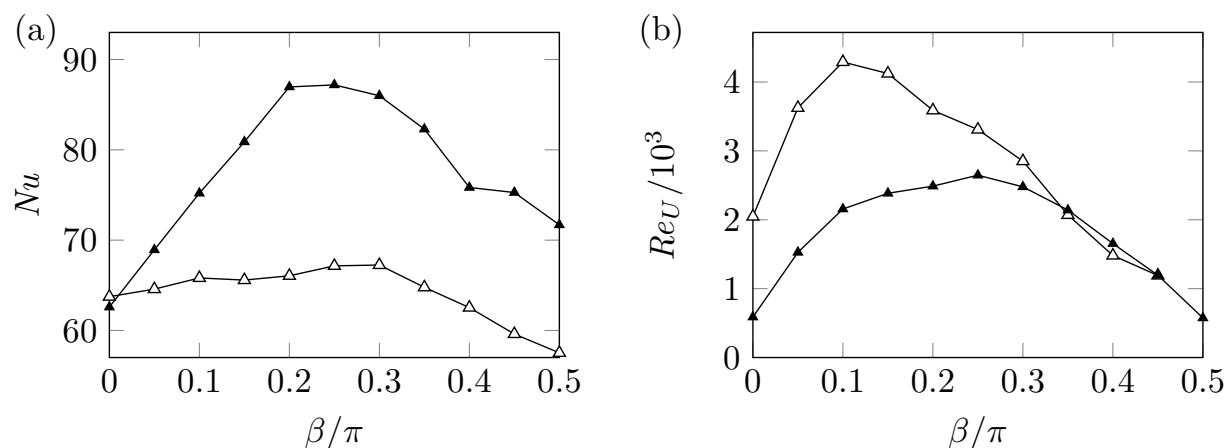

Figure 2.9: The Nusselt number and the different Reynolds numbers for $R a=$ $10^{9}$ and the aspect ratio $\Gamma=1$ (open triangles) and $\Gamma=1 / 5$ (filled triangles) plotted against the inclination angle $\beta$. (a) Absolute Nusselt number. (b) Absolute large-scale Reynolds number $R e_{U}$. 

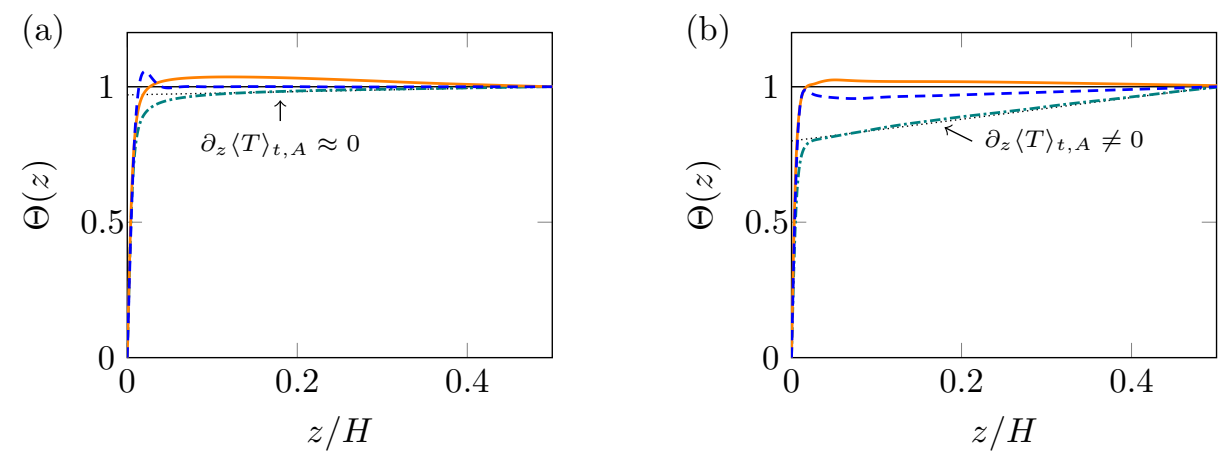

Figure 2.10: The normalised mean temperature profiles, $\Theta(z)=2\left(T_{+}-\right.$ $\left.\langle T\rangle_{t, A}\right) / \Delta$, for $R a=10^{9}, \operatorname{Pr}=1$ and three different inclination angles $\beta / \pi=0$ (green dash-dotted), $\beta / \pi=0.25$ (orange solid) and $\beta / \pi=0.5$ (blue dashed). The aspect ratios are (a) $\Gamma=1$ and (b) $\Gamma=1 / 5$.


(b)


(c)
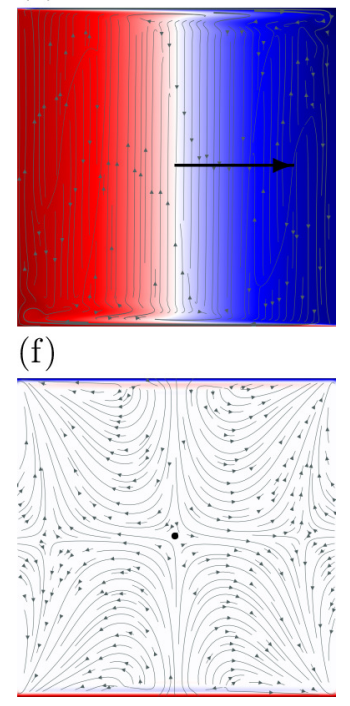

Figure 2.11: Vertical cross-sections of the time-averaged flow fields, for $R a=$ $10^{9}, \operatorname{Pr}=1$ and $\Gamma=1$, through (a-c) the plane of the large scale circulation and (d-f) the plane perpendicular to the LSC. The inclination angles are $(\mathrm{a}, \mathrm{d}) \beta / \pi=0,(\mathrm{~b}, \mathrm{e}) \beta=\pi / 4$ and (c, f) $\beta=\pi / 2$. The thick black arrows indicate the strength and direction of gravity. Colour scale and streamlines as in figure 2.4. 
(a)

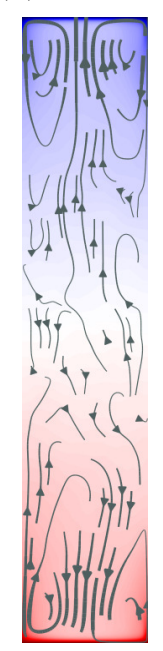

(b)

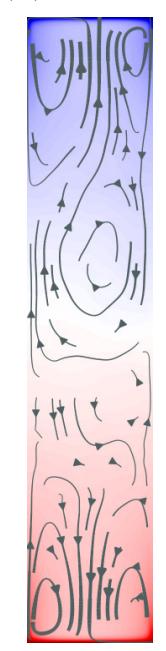

(c)

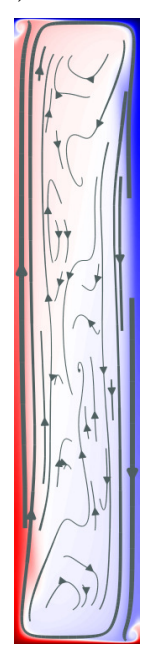

(d)

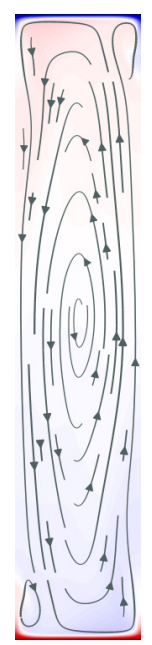

(e)

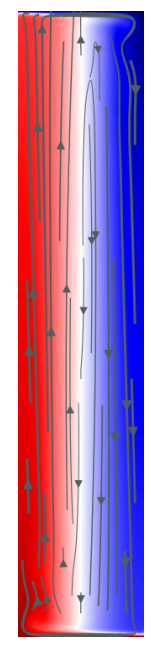

(f)

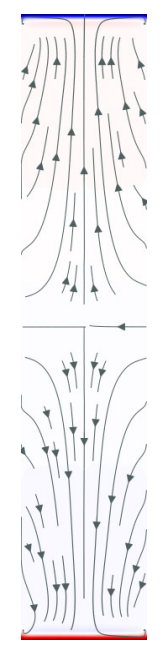

Figure 2.12: Vertical cross-sections of the time-averaged flow fields, for $R a=$ $10^{9}, \operatorname{Pr}=1$ and $\Gamma=1 / 5$, through two perpendicular planes. The inclination angles are $(\mathrm{a}, \mathrm{b}) \beta / \pi=0,(\mathrm{c}, \mathrm{d}) \beta=\pi / 4$ and $(\mathrm{e}, \mathrm{f})$ $\beta=\pi / 2$. Colour scale and streamlines as in figure 2.4.

(a)

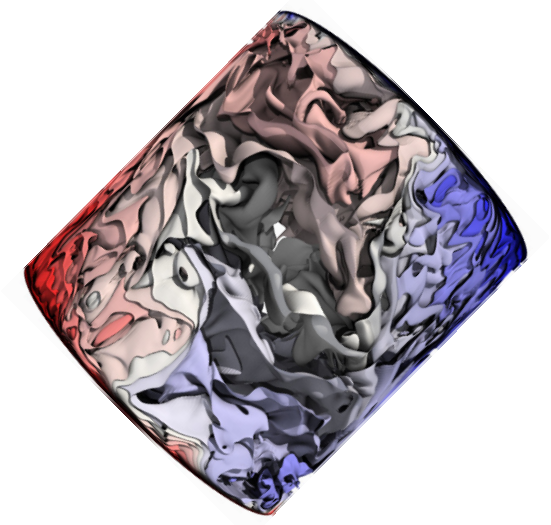

(b)

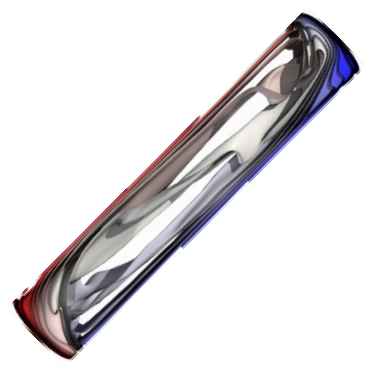

Figure 2.13: Isosurfaces of the instantaneous temperature field for $R a=10^{9}$, $\operatorname{Pr}=1, \beta=\pi / 4$. The aspect ratios are (a) $\Gamma=1$ and (b) $\Gamma=1 / 5$. Colour scale as in figure 2.4. 
states as well, but initiating the simulations from the single-roll state leads to the double-roll state. This suggests that the double-roll state might be the preferred state; however, finding the probability of each state is computationally too expensive. At the Rayleigh number $R a=5 \times 10^{7}$ and higher, there is no long-term LSC present any more. This is caused by the small aspect ratio, as we will discuss in section 2.3.4.

\subsubsection{The effect of confinement}

To investigate the influence of the aspect ratio on the transport quantities, as well as on the flow structure, simulation data for $\operatorname{Ra}=10^{9}, \operatorname{Pr}=1$ and 11 different inclination angles $\beta$ are compared for the aspect ratios $\Gamma=1$ and $\Gamma=1 / 5$. Thereby, a special focus lies on the cases of $\operatorname{RBC}(\beta / \pi=0)$, the optimal inclination angle $\left(\beta=\beta^{*}\right)$ and $\operatorname{VC}(\beta / \pi=0.5)$.

In RBC both aspect ratios have similar values of the Nusselt numbers, where $N u(\Gamma=1)$ is less than $2 \%$ larger than $N u(\Gamma=1 / 5)$. Although the heat transport shows qualitatively similar behaviour with inclination in both cases, i.e. the heat transport increases with inclination, reaches a maximum at a certain inclination angle (in this case $\beta^{*} \approx \pi / 4$ for both aspect ratios) and then decreases again, there are some important differences. The first and most apparent difference is the higher relative increase in the heat transport for the slender cylinder, which is approximately $40 \%$ compared to only $6 \%$ increase for the cylinder with aspect ratio one (figure 2.9a). Furthermore, the smallaspect-ratio cylinder shows a larger heat transport for VC compared to RBC, but for the aspect-ratio-one cylinder, $\mathrm{VC}$ has a lower heat transport compared to the respective $\mathrm{RBC}$.

In contrast to the heat transport, the Reynolds number, based on the kinetic energy, is higher for all inclination angles in aspect ratio $\Gamma=1$, whereas the relative increase with inclination is higher for the slender cylinder. This is due to the stabilizing effect of the slender geometry on the flow. A look at the largescale Reynolds numbers $R e_{U}$ (figure $2.9 \mathrm{~b}$ ) reveals that, in aspect ratio $\Gamma=1$, the maximum is reached already at $\beta=0.1 \pi$, which is at a smaller inclination angle than the optimal one $\left(\beta^{*}=\pi / 4\right)$. Above we discussed that for slender cylinders the points of the maxima of $R e_{U}$ and of the Nusselt number coincide, pointing out that the strength of the LSC is important for the increase in the heat transport (Teimurazov \& Frick, 2017). Also from the same figure we can see that, in aspect ratio one, there is a dominant LSC in RBC.

In figure 2.10 we compare the normalized temperature profiles, averaged in time and over cross-sections at distance $z$ from the heated plate, of the two 
aspect ratios, for the three cases: $\mathrm{RBC}$, the optimal inclination angle and VC. The temperature is normalized in such a way that it is equal to zero at the heated plate and to one in the core part of the domain. The usual expectation of the temperature profile in $\mathrm{RBC}$ is that the temperature rises within the thermal BL close to the mean value, then in the bulk region it takes the mean value, hence the temperature gradient is approximately zero in the bulk. In the aspect-ratio-one case, we observe this expected behaviour, but in the slender cylinder, the temperature rises only to approximately $3 / 4$ of the bulk temperature within the thermal BL. This is accompanied by a non-zero temperature gradient inside the bulk region (see figure 2.10b). At an inclination angle of $\beta=\pi / 4$, both aspect ratios show a slight inversion of the bulk temperature gradient, i.e. the sign of the temperature gradient changes compared to the gradient of the conductive profile. This kind of behaviour was previously observed by Scheel \& Schumacher (2016) for a very low Prandtl number, $\operatorname{Pr}=0.005$, in RBC inside an aspect-ratio-one cylinder. For vertical convection there is a slightly colder/warmer region above the hot/cold thermal BL. These regions show up as local extrema in the temperature profile and can also be seen in the vertical slices of the time-averaged fields (figures 2.11c,f and $2.12 \mathrm{e}, \mathrm{f})$. In the aspect ratio $\Gamma=1$ case, the region of the local extremum shows an inversion, which means that the layer of hot fluid above the BL has a temperature larger than the average temperature inside the bulk.

Thermal plumes are an important feature of turbulent thermal convection and, as mentioned above, the formation of plume columns apparently facilitates an increase of the heat transport. Further visualization of the flow field reveals how thermal plumes are influenced by inclination and the aspect ratio. Slices taken at the edge of the thermal BL, $z=\delta_{\theta}$, of the instantaneous temperature and velocity fields are shown for both aspect ratios in figure $2.7(\mathrm{a}-\mathrm{f})$. There is a visual aid (black circle) given to show the diameter of the small-aspect-ratio cylinder scaled by its height. In the case of RBC and aspect ratio one, plenty of thermal plumes are visible, whereas the aspect ratio $\Gamma=1 / 5$ shows just very few. However, the absolute size of the thermal plumes appears to be similar, since the Rayleigh number and Prandtl number are the same for both aspect ratios. Tilting the aspect-ratio-one cylinder causes the plumes to change their orientation with respect to the mean flow direction: they align perpendicular to the streamlines. On the contrary, the optimally inclined, slender cylinder shows no separate thermal plumes, but only one rising and one falling plume, which span the whole cylinder (cf. figures 2.7b,e and 2.13a,b).

Furthermore, the flow inside the slender cylinder is strongly stratified com- 
pared to the aspect-ratio-one case (cf. figure 2.13). In the case of RBC, buoyancy is the only mechanism to cause convection, but as soon as the cell is tilted, the shear flow becomes important. From inclined layer convection, where the aspect ratio is large or infinite, it is known that these are two different instability mechanisms that govern the fluid motion: one due to buoyancy and the other due to shear. The buoyancy or thermal mechanism leads to the development of longitudinal rolls where the axes are aligned with the shear flow. The onset of the longitudinal rolls is independent of $\mathrm{Pr}$. The other mechanism, i.e. the shear-flow mechanism, determines the development of transverse rolls, the axes of which are transverse to the shear. In the competition between these two mechanisms, the Prandtl number is crucial: for $\operatorname{Pr}<0.26$ transverse rolls are always realized, while for larger $\operatorname{Pr}$ longitudinal rolls may start first if the inclination angle is not too large. This has been studied in various works see Chen \& Pearlstein (1989); Fujimura \& Kelly (1993); Bodenschatz et al. (2000); Daniels et al. (2000, 2003) and references therein. Thus, depending on the aspect ratio, there might be a specific inclination angle at which both mechanisms support the heat transport in such a way that it can explain the strong increase of the heat transport in inclined convection.

Further features of the flow become apparent in the time-averaged fields. These are again taken at the edge of the thermal BL, $z=\delta_{\theta}$, as horizontal slices (figure $2.7 \mathrm{~g}-1$ ). In RBC inside the aspect-ratio-one cell a long-term LSC is present in the flow, as one can see in figures $2.7 \mathrm{~g}$ and $2.11 \mathrm{a}$. In contrast to this, for $\Gamma=1 / 5$, there are no signs of an LSC visible; instead, there are ring vortices close to the plates (figure 2.12a,b). The inclined aspect-ratio-one cell shows a symmetric flow field (figure $2.7 \mathrm{~h}$ ) with two hot channels at each side and a smaller impinging zone compared to $\Gamma=1 / 5$ (figure $2.7 \mathrm{k}$ ). For the latter case we observe that the asymmetric flow from the instantaneous field is still present, despite a long averaging time of $t_{\text {avg }}=1725 T_{f}$. However, the flow is not steady - the velocity is still fluctuating - which means that the asymmetric flow structure prevails for a long time. This asymmetry can be seen in the vertical slice through the plane that is perpendicular to the LSC as well (figure $2.12 \mathrm{~d}$ ). In vertical convection the velocity field in the aspectratio-one case is almost parallel over the whole plate, although one can see the influence of the sidewalls (figure $2.7 \mathrm{c}, \mathrm{f}$ ). In the averaged fields (figures 2.11c,f and $2.12 \mathrm{e}, \mathrm{f})$ we see a difference in the flow organization in the vertical slice that is perpendicular to the LSC. In the small aspect ratio case there is only one saddle point in the velocity vector field, while in the aspect-ratio-one case, there are three saddle points at half height. 
(a)

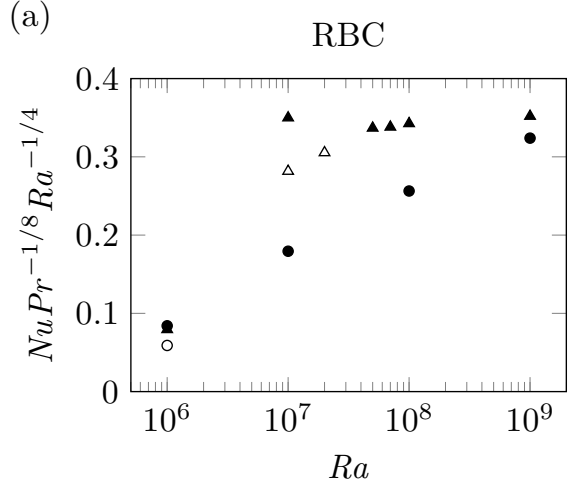

(c)

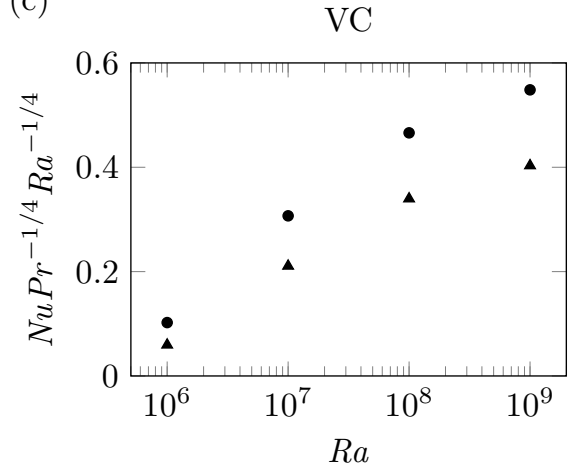

(b)

$\mathrm{RBC}$

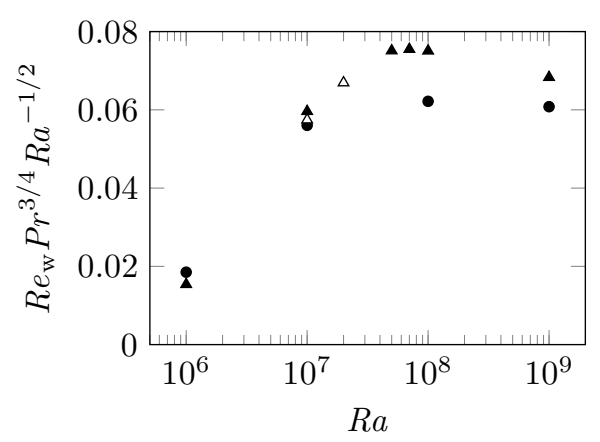

(d)

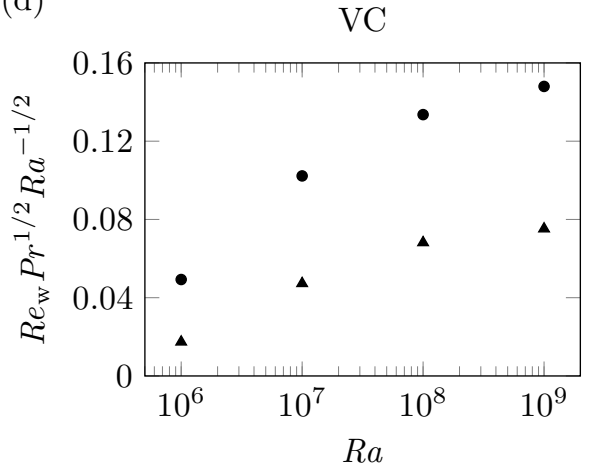

Figure 2.14: (a, c) The Nusselt number and (b, d) the Reynolds number, compensated with respect to the Rayleigh and Prandtl number scaling relations in the regime $\mathrm{I}_{l}(\mathrm{a}, \mathrm{b})$ in $\mathrm{RBC}$ (Grossmann \& Lohse, 2000) and (c, d) in VC (Shishkina, 2016) for Prandtl numbers $\operatorname{Pr}=1$ (triangles) and $\operatorname{Pr}=0.1$ (circles). Flows in a single-roll state are marked with filled symbols and flows in double-roll state are marked with open symbols.

\subsubsection{Limiting cases: Rayleigh-Bénard and vertical convection}

Global heat and momentum transport scaling relations $N u \sim R a^{a} \operatorname{Pr}^{b}$ and $R e \sim R a^{c} \operatorname{Pr}^{d}$, respectively, are of common interest in all configurations of convective flows and here we will briefly discuss the two limiting cases of inclined convection, which are $\operatorname{RBC}(\beta=0)$ and $\operatorname{VC}(\beta=\pi / 2)$. The scaling relations for these cases are presented in figure 2.14 in compensated plots with the scaling exponents according to the boundary-layer-determined lowPrandtl-number regime $\mathrm{I}_{l}$ (Grossmann \& Lohse, 2000; Shishkina, 2016). Here the wind-based Reynolds number (2.7) is used, since the GLT assumes a largescale wind above the BL, which is best represented by the velocity along the plate.

In the regime $\mathrm{I}_{l}$, the kinetic and thermal energy dissipation rates are both dominated by their BL contributions. In the vicinity of the plates, the kinetic $\mathrm{BL}$ is embedded in the thermal one, while the kinetic BL, which is attached 
to the adiabatic sidewall of the cylinder, is located outside the thermal BL. In a slender container, the kinetic BL at the adiabatic sidewall occupies a larger fraction of the total volume and this strongly influences the scaling relations.

In all considered cases we observe the exponents ( $a$ and $c$ ) in the $N u$ vs. $R a$ and in the $R e$ vs. $R a$ scaling relations, which are larger than those predicted for fully developed, unconfined convective flows. The reason for this discrepancy is the strong lateral geometric confinement (small $\Gamma$ ) that shifts the onset of convection to higher critical Rayleigh numbers. Since for fully developed convective flows the scaling exponents $a$ and $c$ should be the same for all $\Gamma$, in the case $\Gamma<1$ there exists a transitional $R a$ region with higher scaling exponents than those predicted by the theory for infinite plates. In other words, for the considered Rayleigh numbers, the size of the heated/cooled plates is not sufficiently large to observe the scaling relations proposed for infinite plates. This kind of transition was observed previously, for example in DNS of RBC inside an aspect-ratio-one cylinder, where the onset of convection and the transition to turbulence in mercury were investigated (cf. figure 11 in Verzicco \& Camussi (1997)). The above discussed influence of the geometrical confinement on the scaling relations is stronger for smaller Rayleigh numbers.

Finally we note that, although the obtained exponent $a$ in the $N u$ vs. $R a$ scaling relation in VC is slightly larger than that proposed in Shishkina (2016) ( $a=1 / 4$ ), it is significantly smaller than that proposed in Castaing et al. (2017) for small-Prandtl-number soft-turbulence regime $(a=2)$ and significantly larger than that proposed in Castaing et al. (2017) for large-Prandtlnumber soft-turbulence regime $(a=1 / 5)$.

\subsection{Summary and conclusions}

A favourable combination of the Rayleigh number, Prandtl number, aspect ratio and inclination angle can lead to a large increase in heat transport compared to classical Rayleigh-Bénard convection. Among the studied cases the highest relative increase of the heat transport was observed for $R a=10^{7}$, $\operatorname{Pr}=0.1$ and an inclination angle of $\beta=\pi / 4$ in an aspect ratio $1 / 5$ cylinder. In this case, the heat transport is increased by a factor of approximately 2.3 .

Changes in the flow structure were identified that lead to the significant increase of the heat transport. First, inside a geometrically confined, slender container there is a stronger enhancement of the LSC, which is caused by the shear flow in inclined convection. The large-scale Reynolds number, $R e_{U}$, can be several times larger in inclined convection compared to RBC. Thus, it increases by a factor of approximately 4.5 at the optimal inclination angle 
compared to no inclination for the slender cylinder and $\operatorname{Ra}=10^{9}$ and $\operatorname{Pr}=1$. In the aspect-ratio-one cylinder, the large-scale Reynolds number increased only by a factor of 1.6, for the same Rayleigh and Prandtl numbers.

Second, inside the slender cylinder with inclination, plumes stack onto each other forming plume columns that eventually reach and impinge on the opposite BL. Since we always observe the optimal heat transport as soon as those plume columns span the whole cylinder, the interaction of the plumes with the opposite BL is certainly important for the heat transport enhancement.

Third, usually at a larger inclination angle than the optimal one, the flow becomes laminar-like or even (almost) steady. We observe this transition to steady flow for all parameters except for the combination of the highest Rayleigh number and the lowest Prandtl number. This transition to steady flow may show oscillatory states, which means that the route to turbulence in inclined convection is similar to that in RBC.

Furthermore, we observe that for lower Prandtl number and/or larger aspect ratio, the optimal heat transport is reached at a larger value of $\beta / \pi$. Careful considerations suggest that this is also the case for higher Rayleigh numbers.

Additionally we observed the existence of single- and double-roll states in $\mathrm{RBC}$ and confirmed that the double-roll state has indeed less efficient heat transport compared to the single-roll state.

Since in a slender geometry, the sidewalls strongly affect the flow, we could not observe the scaling relations expected for convection between infinite plates. Furthermore, the symmetry is broken by the tilt of the cylinder and we observed asymmetric solutions. For turbulent RBC in the slender cylinder we did not find a prevailing LSC as in the aspect-ratio-one cell. However, the presence of the LSC is not that important in RBC, since the heat transport is approximately the same in the $\Gamma=1$ and $\Gamma=1 / 5$ cases. This is in conformity with previous results by Ciliberto et al. (1996). However, it is important to note that, in inclined convection inside small-aspect-ratio cylinders, the LSC appears to play a very important role for the heat transport. Thus, our data suggest that the highest increase in heat transport for a certain aspect ratio depends not only on the inclination angle but also on the Rayleigh number and Prandtl number.

Acknowledgements. This work is supported by the Priority Programme SPP 1881 Turbulent Superstructures of the Deutsche Forschungsgemeinschaft (DFG) under the grant Sh405/7. O.S. thanks DFG for the support under the grant Sh405/4 - Heisenberg fellowship. The authors acknowledge Leibniz Supercomputing Centre (LRZ) for providing computing time. 



\section{Elliptical instability and multi-roll flow modes of the large-scale circulation in confined turbulent Rayleigh-Bénard convection}

This chapter is a reprint of a submitted manuscript, which has been uploaded to a pre-print server (arxiv.org).

LukAs Zwirner, Andreas Tilgner \& Olga Shishkina: Elliptical Instability and Multi-Roll Flow Modes of the Large-scale Circulation in Confined Turbulent Rayleigh-Bénard Convection. arXiv:2002.06951 (2020).

Own contribution: conduction of all direct numerical simulations, data analysis, visualization of the data and writing the first draft of the paper.

Abstract. Turbulent Rayleigh-Bénard convection in slender cells exhibits rich dynamics of the large-scale circulation (LSC), with several rolls stacked on top of each other. We propose that the elliptical instability is the mechanism which causes the twisting and breaking of the LSC into multiple rolls and that the volume-averaged heat and momentum transport, represented by the Nusselt number and Reynolds number, is generally weaker for larger number $n$ of the LSC rolls. This is supported by direct numerical simulations for $R a=5 \times 10^{5}, \operatorname{Pr}=0.1, H=5 D$ and $1 \leq n \leq 4$.

\subsection{Introduction}

In thermally driven flows, one of the most prominent features is the largescale circulation (LSC) of a fluid, which contributes significantly to the heat and mass transport in the system. The capability of the LSC to transport heat and mass is influenced by its shape and rich dynamics. Rayleigh-Bénard convection (RBC), where a fluid is confined between a heated plate (at temperature $T_{+}$) from below and a cooled plate (at temperature $T_{-}$) from above, is a paradigmatic system in thermal convection studies (Bodenschatz et al., 2000; Ahlers et al., 2009; Lohse \& Xia, 2010; Chillà \& Schumacher, 2012); it is characterized by the Rayleigh number, $R a \equiv \alpha g \Delta H^{3} /(\kappa \nu)$ (thermal driving), Prandtl number, $\operatorname{Pr} \equiv \kappa / \nu$ (fluid property), and geometry of the convection cell ${ }^{\ddagger}$.

\footnotetext{
${ }^{\ddagger}$ Here, $\alpha$ is the isobaric thermal expansion coefficient, $\nu$ the kinematic viscosity, $\kappa$ the thermal diffusivity, $g$ the acceleration due to gravity, $\Delta \equiv T_{+}-T_{-}$and $T_{+}$and $T_{-}$are the temperatures of heated and cooled plates.
} 
Although the LSC in RBC has been known for a long time, recent investigations aim to provide a deeper understanding of its versatile dynamics, e.g. reversals, precession, sloshing and twisting (Funfschilling \& Ahlers, 2004; Funfschilling et al., 2008; Xi et al., 2009; Zwirner et al., 2020a). One particular factor that influences the LSC is the geometry of the convection cell. Several studies focused on how lateral confinement in one direction influences the heat transport and flow structures (Wagner \& Shishkina, 2013; Huang et al., 2013; Chong \& Xia, 2016; Chong et al., 2015, 2018), though only a few studies focused on lateral confinement in two directions, e.g. slender cylindrical cells of small diameter-to-height aspect ratio $\Gamma=D / H$. Not only a single-roll mode (SRM) of the LSC, but also a double-roll mode (DRM) - composed of two rolls on top of each other - was found for cylindrical cells with $\Gamma=1,1 / 2$, $1 / 3$ and 1/5 (Xi \& Xia, 2008; Weiss \& Ahlers, 2013; Zwirner \& Shishkina, 2018). Experimental studies with water $(\operatorname{Pr} \approx 5)$ found that the SRM is characterized by a slightly enhanced heat transport $(\approx 0.5 \%)$ compared to the DRM (Xi \& Xia, 2008; Weiss \& Ahlers, 2013). It was also found that smallsystems spend more time in the DRM than in the SRM. Direct Numerical Simulations (DNS) (Zwirner \& Shishkina, 2018) for $R a=10^{6}, \operatorname{Pr}=0.1$ and $\Gamma=1 / 5$ showed that the heat transport of the DRM is only $80 \%$ compared to the SRM.

In 2D DNS (van der Poel et al., 2011, 2012), up to four vertically stacked rolls were found for $\Gamma=0.4$. Less heat transport was observed in the case of more rolls and the comparison at different $\operatorname{Pr}$ revealed that the $\Gamma$-dependence is more pronounced at lower $\operatorname{Pr}$. It remains unclear, however, whether in 3D there exist multi-roll flow modes (with three or more rolls on top of each other), what is their efficiency in heat transport and which mechanism creates these modes.

In this Letter, we explore multi-roll modes of the LSC in RBC and propose the elliptical instability as a plausible mechanism to trigger their formation (Kerswell, 2002). This inertial instability also plays a role, e.g., in the precession-driven motion of the Earth's core (Lorenzani \& Tilgner, 2003) and in the dynamics of vortex pairs (Leweke \& Williamson, 1998; Leweke et al., 2016). In the study (Bars \& Dizes, 2006) of the elliptical instability under an imposed radial temperature gradient, it was found that its growth rate decreases with increasing $R a$, but this effect is less pronounced at low $\mathrm{Pr}$.

Numerical method. We conduct DNS using the high-order finite-volume code GOLDFISH (Kooij et al., 2018), which solves the momentum and energy 
equations in Oberbeck-Bousinessq approximation, for an incompressible flow $(\nabla \cdot \mathbf{u}=0)$ :

$$
\begin{aligned}
\partial_{t} \mathbf{u}+\mathbf{u} \cdot \nabla \mathbf{u} & =-\nabla p+\nu \nabla^{2} \mathbf{u}+\alpha g \theta \hat{\mathbf{z}} \\
\partial_{t} \theta+\mathbf{u} \cdot \nabla \theta & =\kappa \nabla^{2} \theta
\end{aligned}
$$

The DNS were conducted at $R a=5 \times 10^{6}, \operatorname{Pr}=0.1$ and $\Gamma=1 / 5$, using a mesh of $256 \times 128 \times 22$ nodes in $z, \varphi$ and $r$-directions, which is of sufficient resolution (Shishkina et al., 2010; Zwirner \& Shishkina, 2018). We consider the volume-averaged instantaneous heat transport $N u(t) \equiv\left(\left\langle u_{z}(t) \theta(t)\right\rangle-\right.$ $\left.\kappa\left\langle\partial_{z} \theta(t)\right\rangle\right) /(\kappa \Delta / H)$ (Nusselt number) and the Reynolds number, $R e(t) \equiv$ $H\left\langle\mathbf{u}^{2}\right\rangle / \nu$, which is based on the kinetic energy. Here and in the following $\langle\cdot\rangle$ denotes volume average, - time average and $\langle\cdot\rangle_{S}$ horizontal area average.

\subsection{Properties of different flow modes}

Inside the slender cylindrical cell of $\Gamma=1 / 5$, we observe flow modes consisting of up to $n=4$ distinct rolls, which are vertically stacked (Fig. 3.1a-d). These $n$-roll flow modes endure for a few free-fall time units, $t_{f} \equiv H / \sqrt{\alpha g \Delta H}$, before they transition into another mode. From time to time, the SRM is strongly twisted (Fig. 3.1a), before it breaks up into two distinct rolls (Fig. 3.1b). Also, the rolls of the DRM may break up into more rolls or are only twisted for a certain time period. Whether the rolls are twisted or break up can be distinguished by the shape of the profiles along the cylinder axis of different horizontally-averaged quantities, in particular, of the normalized horizontal and vertical components of the squared velocity, $u_{\mathrm{h}}^{2}(t, z) / U^{2}=$ $\left\langle u_{r}^{2}+u_{\varphi}^{2}\right\rangle_{S} / \overline{\langle\mathbf{u} \cdot \mathbf{u}\rangle}$ and $u_{\mathrm{v}}^{2}(t, z) / U^{2}=\left\langle u_{z}^{2}\right\rangle_{S} / \overline{\langle\mathbf{u} \cdot \mathbf{u}\rangle}$, respectively, and of the temperature $\theta(t, z)$. Note that the profiles are averaged over horizontal slices and depend on time $t$ and the vertical coordinate $z$. In Fig. 3.1a-d the profiles are presented next to the corresponding snapshots of the flow modes. Additionally, the enstrophy profiles, $\omega_{i}^{2}(t, z)$, are shown, which will be discussed below. Note, that the profiles $u_{\mathrm{v}}^{2}(z)$ and $u_{\mathrm{h}}^{2}(z)$ of the DRM (Fig. 3.1b) have, respectively, a characteristic local minimum and maximum at the junction of the rolls $(z \approx 3 / 5 H)$ in contrast to the twisted SRM (Fig. 3.1a), where these extrema are absent. Moreover, the temperature profile of the DRM shows a characteristic step-like behaviour at junction height; there, the temperature gradient is locally increased, resembling a thermal boundary layer. These shapes of the vertical profiles are characteristic and independent from the number of rolls. Based on the analysis of these profiles, we developed an 

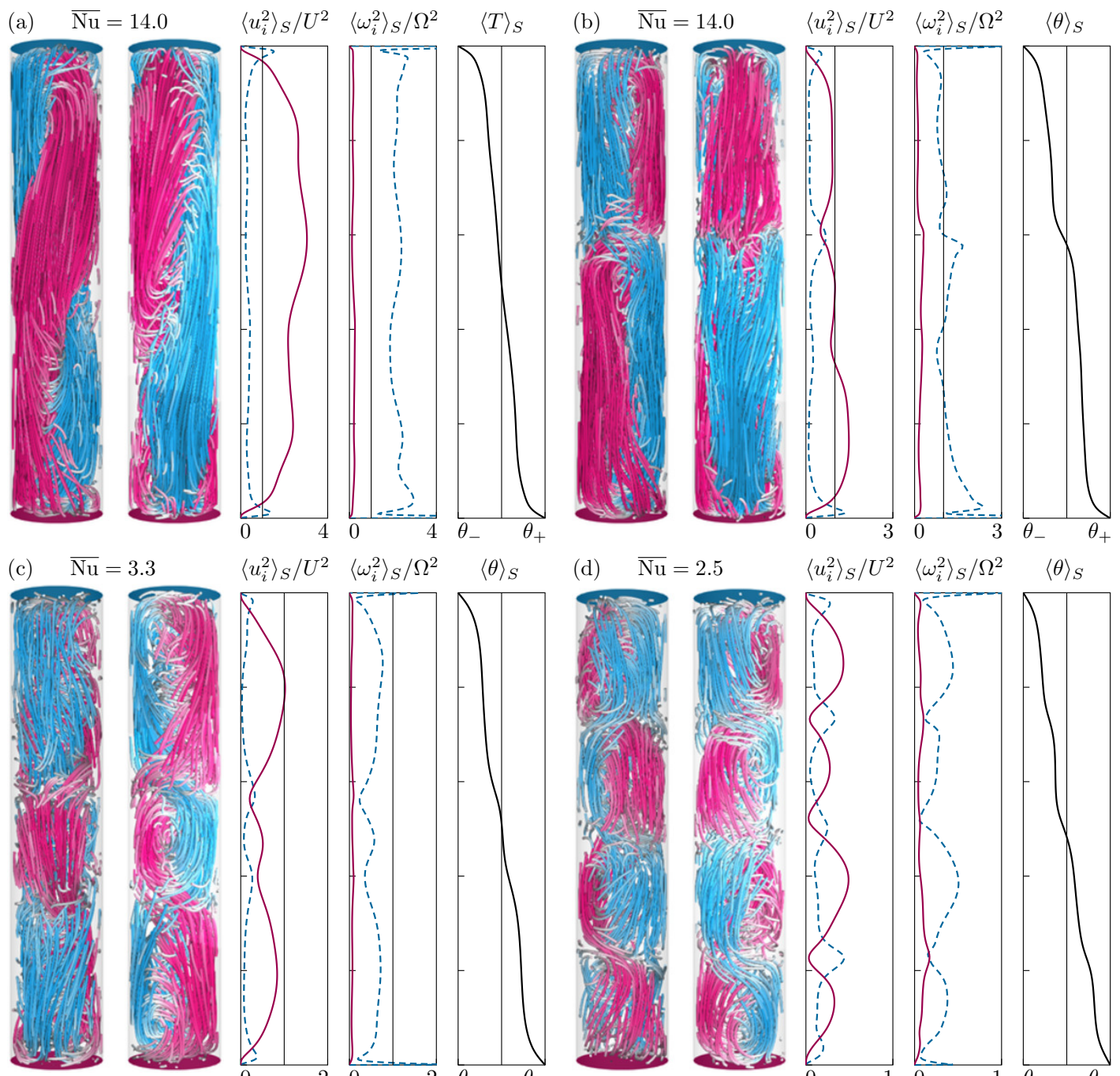
$\left\langle u_{i}^{2}\right\rangle_{S} / U^{2} \quad\left\langle\omega_{i}^{2}\right\rangle_{S} / \Omega^{2}$

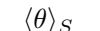

(d) $\quad \overline{\mathrm{Nu}}=2.5$

$\left\langle u_{i}^{2}\right\rangle_{S} / U^{2} \quad\left\langle\omega_{i}^{2}\right\rangle_{S} / \Omega^{2}$

$\langle\theta\rangle_{S}$
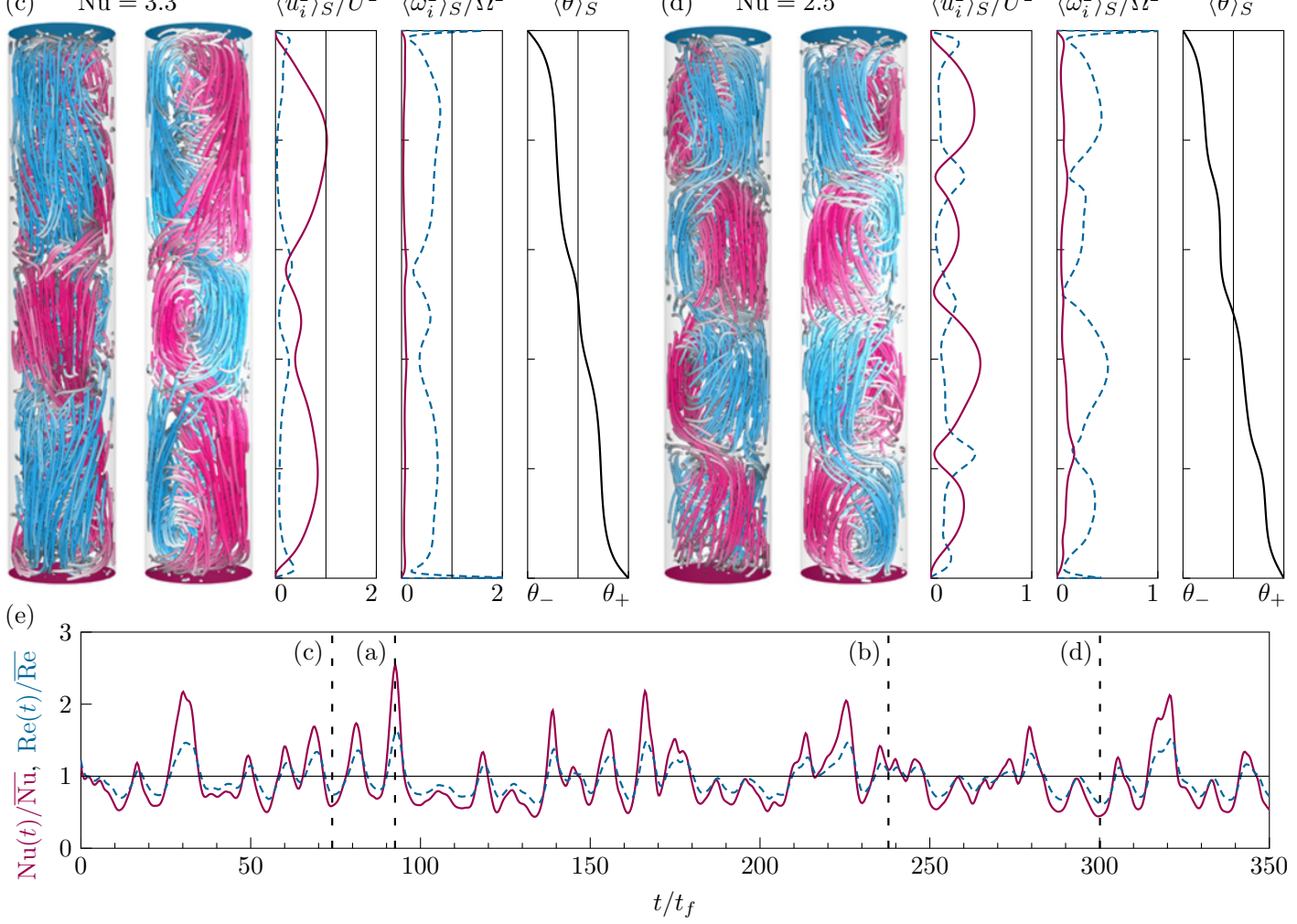

Figure 3.1: Instantaneous flow fields, for a LSC composed of a different number $n$ of rolls: (a) $n=1$, (b) $n=2$, (c) $n=3$, (d) $n=4$. Trajectories of passive tracer particles in two perpendicular perspectives, obtained with the ParaView "Particle Tracer" filter (pink for upward and blue for downward flows), the normalized horizontally averaged profiles of the squared vertical (pink solid) and horizontal (dashed blue) components of the velocity $u_{i}$ and vorticity $\omega_{i}$ and the horizontally averaged profiles of the temperature $\theta$ are shown for each case. (e) Temporal evolution of the normalized volumeaveraged heat flux $N u(t) / \overline{N u}$. The times of the snapshots (a)-(d) are marked by vertical dashed lines. Parameters are: $R a=5 \times 10^{6}$, $\operatorname{Pr}=0.1, \Gamma=1 / 5$. 
algorithm to extract the distinct rolls at any time step and performed conditional averaging on either each $n$-roll flow mode or each roll individually (see supplementary material for details).

Note that the rolls are not necessarily equally distributed within the cell. Thus, two smaller rolls and one larger roll can form a three-roll mode. This is similar to the findings for water (Xi \& Xia, 2008), where a DRM, consisting of a larger roll and a smaller one, was observed. Although one might expect a five-roll mode for the aspect ratio $\Gamma=1 / 5$ as well, such a mode was not observed during the simulated time interval. However, it cannot be excluded that a five-roll mode exists, as it is presumably a rare mode. Note that Xi \& Xia (2008) also did not observe a triple-roll mode in their cell of $\Gamma=1 / 3$.

Furthermore, we examine the enstrophy $\boldsymbol{\omega}^{2}$, which is the squared vorticity, $\boldsymbol{\omega} \equiv \nabla \times \mathbf{u}$, and splits similarly to the squared velocities, into the horizontal, $\omega_{\mathrm{h}}^{2}=\omega_{r}^{2}+\omega_{\varphi}^{2}$ and vertical, $\omega_{\mathrm{v}}^{2}=\omega_{z}^{2}$ contributions. These are normalized with $\Omega^{2}=\overline{\langle\boldsymbol{\omega} \cdot \boldsymbol{\omega}\rangle}$. The horizontal component of the enstrophy profile, $\omega_{h}^{2}(z)$, is strong within the region of a distinct roll and shows a local minimum at the juncture of two rolls and close to the cooled and heated plates (Fig. 3.1a-d). On the other hand, the vertical component of the enstrophy is approximately one order of magnitude weaker (Tab. 3.1).

As discussed above, the vertical profiles allow detection and systematic analysis of all $n$-roll flow modes. One of the primary quantities of interest in a thermally convective system is the global heat transport $(N u)$. Tab. 3.1 lists, among other quantities, the Nusselt number of each flow mode, and it shows that $N u$ decreases as the number of rolls increases. This is a consistent extension of previous studies (Xi \& Xia, 2008; Weiss \& Ahlers, 2013; Zwirner \& Shishkina, 2018), where only SRM and DRM were observed. In contrast to high- $\operatorname{Pr}$ experiments (Xi \& Xia, 2008; Wei \& Xia, 2013), where the difference in the heat transport between the SRM and DRM was only $\approx 0.5 \%$, the decrease of $N u$ in the DRM is apparently much larger $(\approx 30 \%)$ at low $\operatorname{Pr}$.

Besides that, the heat transport also varies strongly in time (Fig. 3.1e), the standard-deviation of $N u$ is 2.6 and the distribution has a strong positive skewness (31.7), which means a long tail at high $N u$. The Reynolds number, $R e$, varies less strongly with time (Fig. 3.1e). The system is most likely to be in a DRM (40.6\%). Additionally, Tab. 3.1 gives the lifetimes, $\tau_{n}$ of each flow mode. The mean lifetime of any flow mode is approximately $2 t_{f}$. 


\begin{tabular}{ccccccc}
$n$ & $\tau_{n} / t_{f}$ & $P_{n} / \%$ & $N u_{n}$ & $R e_{n}$ & $\overline{\left\langle\omega_{\mathrm{h}}^{2}\right\rangle} t_{f}^{2}$ & $\overline{\left\langle\omega_{\mathrm{v}}^{2}\right\rangle} t_{f}^{2}$ \\
\hline 1 & $2.4 \pm 0.4$ & 30.1 & $7.8 \pm 0.3$ & $990 \pm 30$ & $55 \pm 2$ & $7.0 \pm 0.3$ \\
2 & $1.5 \pm 0.2$ & 40.6 & $5.2 \pm 0.3$ & $820 \pm 20$ & $36 \pm 2$ & $5.9 \pm 0.3$ \\
3 & $1.3 \pm 0.2$ & 24.0 & $3.8 \pm 0.3$ & $720 \pm 20$ & $27 \pm 2$ & $5.7 \pm 0.3$ \\
4 & $1.3 \pm 0.4$ & 5.3 & $3.1 \pm 0.3$ & $640 \pm 30$ & $21 \pm 2$ & $4.8 \pm 0.3$ \\
avg & $1.6 \pm 0.1$ & - & $5.5 \pm 0.3$ & $830 \pm 20$ & $39 \pm 2$ & $6.2 \pm 0.3$ \\
\hline
\end{tabular}

Table 3.1: Lifetimes $\tau_{n}$, probabilities $P_{n}$, mean heat transport $N u_{n}$, mean Reynolds number $R e_{n}$, horizontal $\overline{\left\langle\omega_{\mathrm{h}}^{2}\right\rangle}$ and vertical enstrophy $\overline{\left\langle\omega_{\mathrm{v}}^{2}\right\rangle}$, of the $n$-roll flow modes, for $R a=5 \times 10^{6}$, $\operatorname{Pr}=0.1, \Gamma=1 / 5$.
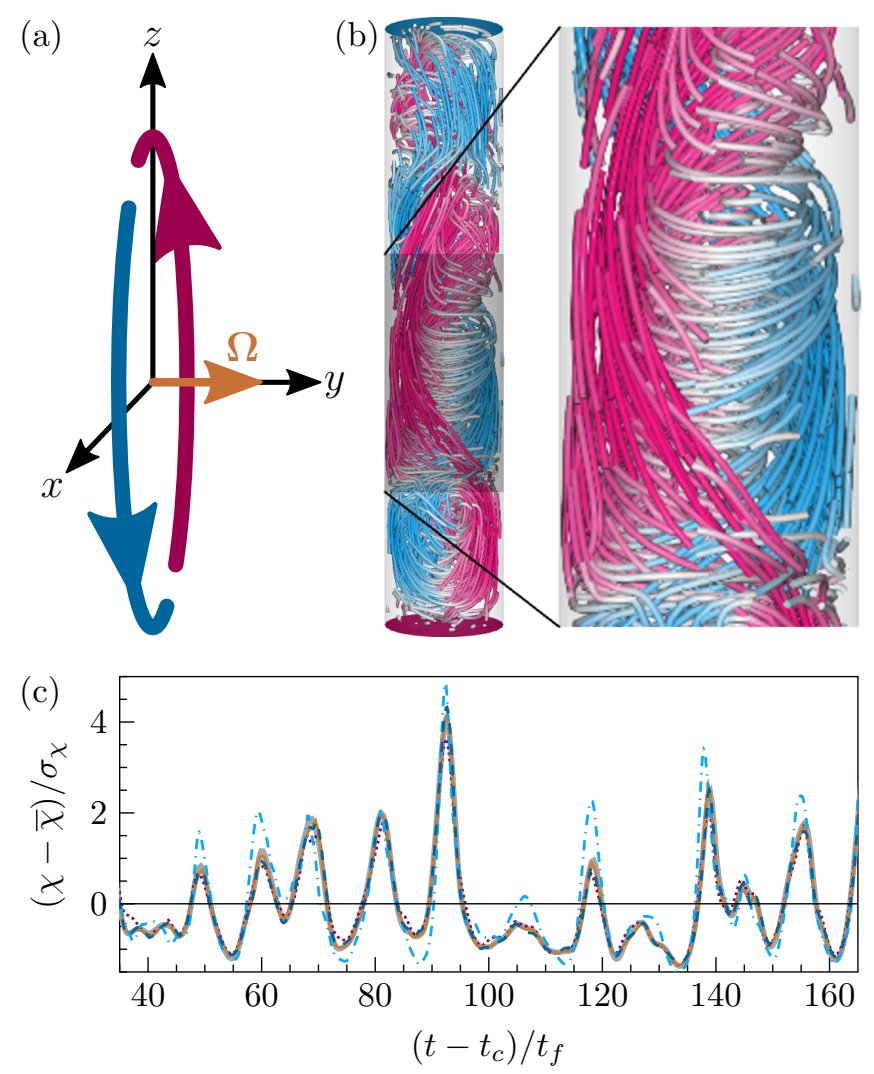

Figure 3.2: (a) Sketch of the primary elliptical LSC, showing the vorticity $\Omega$ of the SRM. (b) A snapshot illustrating a strong azimuthal motion, due to the elliptical instability (colors as in Fig. 3.1a-d). (c) Time signal of the quantity $\chi$, which is either $\langle N u\rangle(t)$ (grey thick solid line), $\left\langle\omega_{\mathrm{h}}^{2}\right\rangle(t)$ (dark blue dashed line), $\left\langle\omega_{v}^{2}\right\rangle(t)$ (light blue dasheddotted line), $\left\langle\varepsilon_{u}\right\rangle(t)$ (red dotted line) or $\left\langle\varepsilon_{\theta}\right\rangle(t)$ (brown thin solid line). Each signal is shifted by its correlation time $t_{c}$ with respect to $\langle N u\rangle(t)$. From each quantity $\chi$ the respective mean value $\bar{\chi}$ is subtracted and then normalized by its standard deviation $\sigma_{\chi}$. DNS for $R a=5 \times 10^{6}, \operatorname{Pr}=0.1, \Gamma=1 / 5$. 


\subsection{Mechanism of the mode transitions}

The elliptical instability refers to the linear instability mechanism that arises from 2D elliptical streamlines and generates a 3D flow (Kerswell, 2002). In its simplest form, the elliptical instability appears for an unbounded strained vortex in inviscid flow, $\mathbf{U}=(\xi-\eta) z \hat{\mathbf{x}}-(\xi+\eta) x \hat{\mathbf{z}}$, where $\hat{\mathbf{x}}$ and $\hat{\mathbf{z}}$ are the unit vectors in $x$ and $z$ directions, respectively (Fig. 3.2a). The strain is denoted by $\eta$ and this vortex has a constant vorticity $\Omega=2 \xi \hat{\mathbf{y}}$ and is characterized by the aspect ratio $\Gamma=\sqrt{(\xi-\eta) /(\xi+\eta)}$. Since the SRM in a slender cylindrical cell resembles such an elliptical vortex (Fig. 3.1a), this instability presumably triggers its break up, and thus the emergence of the multi-roll flow modes. Assuming that the interior of the LSC is nearly isothermal, the stability analysis of the LSC is identical to the stability analysis of an elliptical vortex (Waleffe, 1990; Landman \& Saffman, 1987). The unstable mode contains vorticity along the $z$-direction. Thus, an indicator of the elliptical instability is the growth of vorticity in the direction orthogonal to the vorticity of the elliptical flow, $\boldsymbol{\Omega}$. In Fig. 3.2b a snapshot of the trajectories of passive tracer particles is shown, and a prominent azimuthal flow is visible, which twists and/or breaks up the single-roll LSC. A necessary requirement for the elliptical instability to emerge is that the growth rate, $\sigma$, is much larger than the damping rate due to viscous dissipation, which is of the order $\nu / H^{2}$. To estimate $\sigma$, it is assumed that the aspect ratio of the elliptical SRM is the same as that of the cell, hence $\Gamma=1 / 5$. The vorticity, $2 \xi$, of the SRM is approximated by taking the square root of the averaged horizontal enstrophy $\sqrt{\overline{\left\langle\omega_{h}^{2}\right\rangle}} \approx 7 / t_{f}$ (TAB. 3.1). The inviscid growth rate for the aspect ratio $1 / 5$ is then approximated as $\sigma \approx 0.3 \xi$ (Fig. 1 in Landman \& Saffman (1987)) or $\sigma \approx 1 / t_{f}$. However, the viscous damping is $\nu / H^{2} \approx 1.4 \times 10^{-4} / t_{f}$ and thus about four orders of magnitude smaller than the growth rate. Therefore, the elliptical instability is strong enough to grow.

In $\mathrm{RBC}$, the following relationships of the energy dissipation rates and $\overline{N u}$ hold: $\overline{\left\langle\varepsilon_{u}\right\rangle}=\nu \overline{\left\langle\omega^{2}\right\rangle}=\nu^{3} H^{-4} \operatorname{RaPr}^{-2}(\overline{N u}-1)$ and $\overline{\left\langle\varepsilon_{\theta}\right\rangle}=\kappa \Delta^{2} H^{-2} \overline{N u}$. Although, these equations are only fulfilled for the time averaged quantities, their respective time series are highly correlated as well. An example from these time series and their shift can be seen in Fig. 3.2c. This temporal correlation also holds, if one considers the vertical and horizontal enstrophy components separately. Here, we calculate the correlations in time with respect to $N u(t)$ to find the temporal sequence of the underlying processes. An increase of $N u(t)$ is followed by an increase of the kinetic energy or $R e(t)$ approximately $0.55 t_{f}$ later. After that, the thermal dissipation rate, $\varepsilon_{\theta}$, increases $\left(\approx 0.76 t_{f}\right.$ later $)$. Shortly after that, the horizontal enstrophy, $\omega_{\mathrm{h}}^{2}$, increases 
$\left(\approx 0.84 t_{f}\right.$ later $)$, which is due to the strengthening of the LSC. Finally, the vertical enstrophy, $\omega_{\mathrm{v}}^{2}$, increases $\left(\approx 2 t_{f}\right.$ later $)$, which is presumably caused by the elliptical instability. The delay of $\approx 2 t_{f}$ is, compared to the mean lifetime, $\tau_{n}$, of a $n$-roll flow mode (Tab. 3.1), of similar duration. Note, that the kinetic energy dissipation rate, $\varepsilon_{u}$, which is the sum of the horizontal and vertical enstrophy, has a correlation time of $\approx 1 t_{f}$ which lies, as expected, in between the correlation times of each component. The average time period of the fluctuations of $N u(t)$ is $T_{N u} \approx 12 t_{f}$, hence, the elliptical instability arises delayed by $\approx T_{N u} / 6$. During one period, the LSC can undergo several mode transitions. This demonstrates the temporal interplay of the heat transport, circulation strength and growth of the instability.

\subsection{Conclusions}

It was found that in laterally confined RBC flow modes with more than two rolls stacked on top of each can form. Inside a cylindrical cell of the aspect ratio $\Gamma=1 / 5$ up to four rolls develop for $\operatorname{Pr}=0.1$ and $R a=5 \times 10^{6}$. Based on our long-term DNS, we found that the LSC, which consists of more rolls, transports heat less efficiently. The emergence of the multi-roll flow modes is presumably caused by the elliptical instability.

Acknowledgements. This work is supportet by the Priority Programme SPP 1881 "Turbulent Superstructures" of the Deutsche Forschungsgemeinschaft (DFG) under the grant Sh405/7. The authors acknowledge the Leibniz Supercomputing Centre (LRZ) for providing computing time.

\subsection{Supplementary material: roll extraction algorithm}

The algorithm to extract the different rolls is based on the profiles of the horizontal and vertical profiles of the squared velocities, $u_{z}^{2}(z, t)$ and $u_{h}^{2}(z, t)=$ $u_{\phi}^{2}+u_{r}^{2}$, respectively. It works as follows: First, we calculate $d_{u}(z, t)=u_{z}^{2}-u_{h}^{2}$ and find all zeros $z_{j}^{*}$ of this profile $d_{u}\left(z, t=t_{i}\right)=0$ for every time step $t_{i}$. Second, we assume the junction of two rolls to be located in between two zeros of $d_{u}$, i.e. $z_{n}^{\text {junc. }}=\left(z_{j}^{*}+z_{j+1}^{*}\right) / 2$ and furthermore $d_{u}\left(z_{n}^{\text {junc. }}, t=t_{i}\right)<0$ (the horizontal velocity must be larger than the vertical velocity).

To avoid some difficulties with small "wiggles" in the profiles we restrict the roll size to be $>H / 6$. This restricts the maximum number of rolls to be 5 , however we never observe a 5 -roll state and thus this restrictions is considered reasonable. See Fig. 3.3 to see example extraction of the roll junctions. 


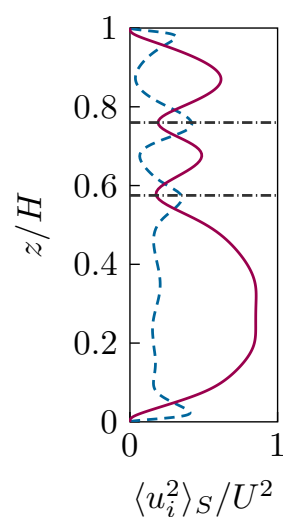

Figure 3.3: Example of how the algorithm works. It shows the profiles $u_{z}^{2}(z, t=$ $\left.t_{i}\right)$ (red solid line) and $u_{h}^{2}\left(z, t=t_{i}\right)$ (blue dashed line). The extraction of the roll junctions are marked (black dash-dotted lines). 



\section{The influence of the cell inclination on the heat transport and large-scale circulation in liquid metal convection}

This chapter is a reprint of the following publication:

L. Zwirner, R. Khalilov, I. Kolesnichenko, A. Mamykin, S. Mandrykin, A. Pavlinov, A. Shestakov, A. Teimurazov, P. Frick \& O. Shishrina: The influence of the cell inclination on the heat transport and large-scale circulation in liquid metal convection. J. Fluid Mech. 884, A18, (2020). Reproduced with permission.

Own contribution: conduction of all direct numerical simulations, analysis of the data from direct numerical simulations and comparison with the data from large-eddy simulations and experiments, writing the first draft of the paper (excluding the sections 4.2.1 and 4.2.3 about the experimental methods and large-eddy simulations).

Abstract. Inclined turbulent thermal convection in liquid sodium is studied at large Rayleigh numbers $R a \gtrsim 10^{7}$ based on the results of both experimental measurements and high-resolution numerical simulations. For a direct comparison, the considered system parameters are set to be similar: $R a=1.67 \times 10^{7}$ in the direct numerical simulations (DNS), $R a=1.5 \times 10^{7}$ in the large-eddy simulations and $R a=1.42 \times 10^{7}$ in the experiments, while the Prandtl number of liquid sodium is very small $(\operatorname{Pr} \approx 0.009)$. The cylindrical convection cell has aspect ratio one; one circular surface is heated, while the other one is cooled. Additionally, the cylinder is inclined with respect to gravity and the inclination angle varies from $\beta=0^{\circ}$, which corresponds to Rayleigh-Bénard convection (RBC), to $\beta=90^{\circ}$, as in a vertical convection (VC) set-up. Our study demonstrates quantitative agreement of the experimental and numerical results, in particular with respect to the global heat and momentum transport, temperature and velocity profiles, as well as the dynamics of the large-scale circulation (LSC). The DNS reveal that the twisting and sloshing of the LSC at small inclination angles periodically affects the instantaneous heat transport (up to $\pm 44 \%$ of the mean heat transport). The twisted LSC is associated with a weak heat transport, while the sloshing mode that brings together the hot and cold streams of the LSC is associated with a strong heat transport. The experiments show that the heat transport scales with Rayleigh number as $N u \sim R a^{0.22}$ in both limiting cases (RBC and VC) for Rayleigh numbers 
around $R a \approx 10^{7}$, while any inclination of the cell, $0<\beta \leq 90^{\circ}$, leads to an increase of the $N u$.

\subsection{Introduction}

Elucidation of the mechanisms of turbulent thermal convection in very-lowPrandtl-number fluids is crucial for our understanding of the universe and the advancement of cooling technology. Turbulent thermal convection takes place, for example, on the surfaces of stars, where the Prandtl number $(P r)$ varies from $10^{-8}$ to $10^{-4}$ (Spiegel, 1962; Hanasoge et al., 2016). Furthermore, turbulent thermal convection in liquid metals $(\operatorname{Pr} \ll 1)$ is relevant in engineering applications, especially in cooling systems of tokamaks and fast breeder reactors (Zhilin et al., 2009; Belyaev et al., 2013). Liquid sodium is of particular interest because of its very low Prandtl number $(\operatorname{Pr} \approx 0.009)$ and it is widely used as cooling agent in fast neutron reactors (Heinzel et al., 2017).

One classical model of thermal convection is Rayleigh-Bénard convection (RBC), where the fluid is confined between a heated lower plate and a cooled upper plate, and the main driving force is buoyancy. The temperature inhomogeneity varies the fluid density, which in presence of gravity leads to the convective fluid motion. For reviews on RBC we refer to Bodenschatz et al. (2000); Ahlers et al. (2009); Lohse \& Xia (2010); Chillà \& Schumacher (2012).

Thermal convection inevitably arises in the case of a horizontal temperature gradient. This is known as vertical convection (VC), convection in cavities or side-heated convection. In vertical convection, the heated and cooled plates are located parallel to the gravity vector and shear plays the key role, see $\mathrm{Ng}$ et al. (2015, 2017); Shishkina (2016).

The concept of inclined convection (IC) is a generalisation of RBC and VC, i.e. the fluid layer between the parallel plates is tilted with respect to the direction of gravity, and both buoyancy and shear act on the flow. This type of convection was studied previously by Daniels et al. (2003); Chillà et al. (2004); Sun et al. (2005); Ahlers et al. (2006b); Riedinger et al. (2013); Weiss \& Ahlers (2013); Langebach \& Haberstroh (2014) and more recently by Frick et al. (2015); Mamykin et al. (2015); Vasil'ev et al. (2015b); Kolesnichenko et al. (2015); Shishkina \& Horn (2016); Teimurazov \& Frick (2017); Mandrykin \& Teimurazov (2019); Khalilov et al. (2018); Zwirner \& Shishkina (2018).

In thermal convection, the global flow structures and heat and momentum transport are determined mainly by the following system parameters: the Rayleigh number $R a$, the Prandtl number $\operatorname{Pr}$ and the aspect ratio of the con- 
tainer $\Gamma$. These are defined as

$$
R a \equiv \alpha g \Delta L^{3} /(\kappa \nu), \quad \operatorname{Pr} \equiv \nu / \kappa, \quad \Gamma \equiv D / L, \quad(4.1 a-c)
$$

respectively. Here $\alpha$ denotes the isobaric thermal expansion coefficient, $\nu$ the kinematic viscosity, $\kappa$ the thermal diffusivity of the fluid, $g$ the acceleration due to gravity, $\Delta \equiv T_{+}-T_{-}$the difference between the temperatures at the heated plate $\left(T_{+}\right)$and at the cooled plate $\left(T_{-}\right), L$ the distance between the plates and $D$ the diameter of the plates.

The main response characteristics of a natural convective system are the mean total heat flux across the heated/cooled plates, $q$, normalised by the conductive part of the total heat flux, $\hat{q}$, i.e. the Nusselt number $N u$, and the Reynolds number Re:

$$
N u \equiv q / \hat{q}, \quad R e \equiv L U / \nu
$$

Here $U$ is the reference velocity, which is usually determined by either the maximum of the time-averaged velocity along the plates or by $\langle\mathbf{u} \cdot \mathbf{u}\rangle^{1 / 2}$, i.e. it is based on the mean kinetic energy; $\mathbf{u}$ is the velocity vector field and $\langle\cdot\rangle$ denotes the average in time and over the whole convection cell. Note that, even for a fixed set-up in natural thermal convection with no additional shear imposed on the system, the scaling relations of the mean heat and momentum transport, represented by $N u$ and $R e$, with the input parameters $R a$ and $P r$, are not universal and are influenced by non-Oberbeck-Boussinesq (NOB) effects, see Kraichnan (1962); Grossmann \& Lohse (2000); Ahlers et al. (2006a, 2009); Lohse \& Xia (2010); Shishkina et al. (2016a,b); Weiss et al. (2018).

Here, one should note that, apart from $\operatorname{Pr}$ and $R a$, the geometrical confinement of the convection cell also determines the strength of the heat transport (Huang et al., 2013; Chong et al., 2015; Chong \& Xia, 2016). Thus, in experiments by Huang et al. (2013) for $\operatorname{Pr}=4.38$, an increase of $N u$ due to the cell confinement was obtained, while in the Direct Numerical Simulations (DNS) by Wagner \& Shishkina (2013) for $\operatorname{Pr}=0.786$, the heat and mass transport gradually reduced with increasing confinement. This virtual contradiction was recently resolved in Chong et al. (2018). It was found that $\operatorname{Pr}$ determines whether the optimal $\Gamma$, at which the maximal heat transport takes place, exists or not. For $\operatorname{Pr}>0.5\left(R a=10^{8}\right)$ an enhancement of $N u$ was observed, where the optimal $\Gamma$ decreases with increasing $\operatorname{Pr}$, but for $\operatorname{Pr} \leq 0.5$ a gradual reduction of the heat transport with increasing confinement was obtained. For all $\operatorname{Pr}$, the confinement induced friction causes a reduction of Re. 
In the general case of inclined thermal convection, the cell inclination angle $\beta\left(\beta=0^{\circ}\right.$ in $\mathrm{RBC}$ and $\beta=90^{\circ}$ in $\left.\mathrm{VC}\right)$ is an influential input parameter of the convective system, apart from $R a, P r$ and the geometry of the container. Experimental studies of inclined thermal liquid-sodium convection in cylinders of different aspect ratios, showed that the convective heat transfer between the heated and cooled surfaces of the container is most efficient neither in a standing position of the cylinder $\left(\beta=0^{\circ}\right)$, nor in a lying position $\left(\beta=90^{\circ}\right)$, but at an inclined position for a certain intermediate value of $\beta, 0^{\circ}<\beta<90^{\circ}$, see Vasil'ev et al. (2015b) for $L=20 D$, Mamykin et al. (2015) for $L=5 D$ and Khalilov et al. (2018) for $L=D$. Moreover, these experiments showed that for $\operatorname{Pr} \ll 1$ and $R a \gtrsim 10^{9}$, any tilt $\beta, 0^{\circ}<\beta \leq 90^{\circ}$, of the cell leads to a larger mean heat flux $(N u)$ than in $\mathrm{RBC}$, at similar values of $R a$ and $\operatorname{Pr}$. Note that the effect of the cell tilting on the convective heat transport in low- $\operatorname{Pr}$ fluids is very different from that in the case of large $\operatorname{Pr}$ (Shishkina \& Horn, 2016). For example, for $\operatorname{Pr} \approx 6.7$ and $R a \approx 4.4 \times 10^{9}$, a monotonic reduction of $N u$ with increasing $\beta$ in the interval $\beta \in\left[0^{\circ}, 90^{\circ}\right]$ takes place, as it was obtained in measurements by Guo et al. (2015).

One should mention that there are only a few experimental and numerical studies of IC in a broad range of $\beta$, whereas most of the investigations of the cell-tilt effects on the mean heat transport were conducted in a narrow region of $\beta$ close to $0^{\circ}$ and mainly for large- $\operatorname{Pr}$ fluids. These studies showed generally a small effect of $\beta$ on $N u$, reflected in a tiny reduction of $N u$ with increasing $\beta$ close to $\beta=0^{\circ}$, see Ciliberto et al. (1996); Cioni et al. (1997); Chillà et al. (2004); Sun et al. (2005); Ahlers et al. (2006b); Roche et al. (2010); Wei \& Xia (2013). A tiny local increase of $N u$ with a small inclination of the RBC cell filled with a fluid of $\operatorname{Pr}>1$ is possible only when a two-roll form of the global large-scale circulation (LSC) is present in RBC, which usually almost immediately transforms into a single-roll form of the LSC with any inclination (Weiss \& Ahlers, 2013). The single-roll LSC is known to be more efficient in the heat transport than its double-roll form, as was proved in the measurements (Xi \& Xia, 2008; Weiss \& Ahlers, 2013) and DNS (Zwirner \& Shishkina, 2018).

The IC in a broad range of the inclination angle $\beta$ (from $0^{\circ}$ to $90^{\circ}$ ) in liquid sodium has been studied so far by Frick et al. (2015); Mamykin et al. (2015); Vasil'ev et al. (2015b); Kolesnichenko et al. (2015); Khalilov et al. (2018). These sodium experiments were conducted in relatively long cylinders, in which the scaling exponents are essentially increased due to the geometrical confinement. For RBC in a cylinder with $L=5 D$, Frick et al. (2015) reported $N u \sim R a^{0.4}$ and for RBC in a very long cylinder with $L=20 D$, Mamykin et al. 
(2015) obtained $N u \sim R a^{0.77}$. In both studies, also IC in liquid sodium for $\beta=45^{\circ}$ was investigated, and the following scaling laws were obtained for this inclination angle: $N u \sim R a^{0.54}$ for $L=5 D$ and $N u \sim R a^{0.7}$ for $L=20 D$. Note that in both IC measurements, much higher mean heat fluxes were obtained, compared to those in $\mathrm{VC}$ or $\mathrm{RBC}$ configurations.

Thus, all available experimental and numerical results on IC show that the $N u(\beta) / N u(0)$ dependence is a complex function of $R a, \operatorname{Pr}$ and $\Gamma$, which cannot be represented as a simple combination of their power functions.

An analogy can be seen between the IC flows and convective flows, which occur from the imposed temperature differences at both the horizontal and vertical surfaces of a cubical container. With a different balance between the imposed horizontal and vertical temperature gradients, where the resulting effective temperature gradient has non-vanishing horizontal and vertical components, one can mimic the IC flows at different inclination angles. Experimental studies on these type of convective flows were conducted by Zimin et al. (1982).

Although there is no scaling theory for general IC, for the limiting configurations of $\mathrm{IC}\left(\beta=0^{\circ}\right.$ and $\left.\beta=90^{\circ}\right)$ and sufficiently wide heated/cooled plates, there are theoretical studies of the scaling relations of $N u$ and $R e$ with $\operatorname{Pr}$ and Ra. For RBC $(\beta=0)$, Grossmann \& Lohse (2000, 2001, 2011) (GL) developed a scaling theory which is based on a decomposition into boundary-layer (BL) and bulk contributions of the time- and volume-averaged kinetic $\left(\epsilon_{u}\right)$ and thermal $\left(\epsilon_{\theta}\right)$ dissipation rates, for which analytical relations with $N u, \operatorname{Ra}$ and $\operatorname{Pr}$ exist. Equating $\epsilon_{u}$ and $\epsilon_{\theta}$ to their estimated either bulk or BL contributions and employing in the BL dominated regimes the Prandtl-Blasius BL theory (Prandtl, 1905; Blasius, 1908; Landau \& Lifshitz, 1987; Schlichting \& Gersten, 2000), theoretically possible limiting scaling regimes were derived. The theory allows us to predict $N u$ and $R e$ in RBC if the pre-factors fitted with the latest experimental and numerical data are used, see Stevens et al. (2013) and Shishkina et al. (2017).

In the other limiting case of $\mathrm{IC}$, which is $\mathrm{VC}\left(\beta=90^{\circ}\right)$, the mean kinetic dissipation rate $\epsilon_{u}$ cannot be derived analytically from $R a, \operatorname{Pr}$ and $N u$, and this impedes an extension of the GL theory to VC. However, for the case of laminar free convection between two differentially heated plates (i.e. VC), it is possible to derive the dependences of $R e$ and $N u$ on $R a$ and $P r$ from the BL equations, under the assumption that a similarity solution exists (Shishkina, 2016). Although this problem is solved for the laminar case, to our knowledge, there is no theoretical model to predict $N u$ and $R e$ in turbulent VC. It 
is expected, however, that in the asymptotic regime of high $R a$, the scaling exponents in the $N u$ versus $R a$ and $R e$ versus $R a$ scalings is $1 / 2$, as in RBC (Ng et al., 2018).

Generally, the dependences of $N u$ and $R e$ on $R a$ and $P r$ in VC have been less investigated than those in RBC. For similar cell geometry and ranges of $R a$ and $\mathrm{Pr}$, not only the heat transport in VC differs quantitatively from that in RBC (Bailon-Cuba et al., 2012; Wagner \& Shishkina, 2013, 2015; Ng et al., 2015), but the $\mathrm{VC}$ and $\mathrm{RBC}$ flows can even be in different states. For example, for $\operatorname{Pr}=1, R a=10^{8}$ and a cylindrical container of $\Gamma=1$, the VC flow is steady, while the RBC flow is already turbulent (Shishkina \& Horn, 2016). Previous experimental and numerical studies of free thermal convection under an imposed horizontal temperature gradient (i.e. VC) reported the scaling exponent $\gamma$ in the power law $N u \sim R a^{\gamma}$, varying from $1 / 4$ to $1 / 3$. In laminar VC, it is approximately 1/4 (Schmidt \& Beckmann, 1930; Lorenz, 1934; Saunders, 1939; Churchill \& Chu, 1975), being slightly larger for very small $R a$, where the geometrical cell confinement influences the heat transport (Versteegh \& Nieuwstadt, 1999; Yu et al., 2007; Kis \& Herwig, 2012; Ng et al., 2015). The scaling exponent $\gamma$ is also larger for very high $R a$, where, with growing $R a$, the VC flows become first transitional (Ng et al., 2017) and later on fully turbulent (George \& Capp, 1979; Fujii et al., 1970). Note that all the mentioned experiments and simulations of $\mathrm{VC}$ were conducted for fluids of $\mathrm{Pr}$ about or larger than 1 .

For the case of small $P r$, in the experiments by Frick et al. (2015) and Mamykin et al. (2015) on turbulent VC in liquid sodium $(\operatorname{Pr} \approx 0.01)$ in elongated cylinders, significantly larger scaling exponents were observed, due to the geometrical confinement. Thus, for a cylinder with $L=5 D$ and the Rayleigh numbers, based on the cylinder diameter, up to $10^{7}$, Frick et al. (2015) obtained $N u \sim R a^{0.43}$ and $R e \sim G r^{0.44}$, where $G r$ is the Grashof number, $G r \equiv R a / P r$. For an extremely strong geometrical confinement, namely, for a cylindrical convection cell with $L=20 D$, and a similar Rayleigh number range, Mamykin et al. (2015) found $N u \sim R a^{0.95}$ and $R e \sim G r^{0.63}$.

There exist a few measurements of the scaling relations of $N u$ versus $R a$ in liquid-metal Rayleigh-Bénard convection (without any cell inclination). For mercury $(\operatorname{Pr} \approx 0.024)$, it was reported $N u \sim R a^{0.27}$ for $2 \times 10^{6}<R a<8 \times 10^{7}$ by Takeshita et al. (1996), $N u \sim R a^{0.26}$ for $7 \times 10^{6}<R a<4.5 \times 10^{8}$ and $N u \sim R a^{0.20}$ for $4.5 \times 10^{8}<R a<2.1 \times 10^{9}$ by Cioni et al. (1997) and $N u \sim R a^{0.29}$ for $2 \times 10^{5}<R a<7 \times 10^{10}$ by Glazier et al. (1999). For liquid gallium $(\operatorname{Pr} \approx 0.025)$, King \& Aurnou (2013) measured $N u \sim R a^{0.25}$ 
for $2 \times 10^{6}<R a<10^{8}$, and for GaInSn $(P r=0.029)$, Zürner et al. (2019) measured $N u \sim R a^{0.27}$ for $4 \times 10^{6}<R a<6 \times 10^{7}$. For liquid sodium $(\operatorname{Pr}=$ 0.006), Horanyi et al. (1999) measured $N u \sim R a^{0.25}$ for $2 \times 10^{4}<R a<5 \times 10^{6}$.

Conducting accurate DNS of natural thermal convection at high $R a$ and very low $\operatorname{Pr}$ is very challenging, since it requires very fine meshes in space and short steps in time due to the necessity to resolve the Kolmogorov microscales in the bulk of the flows as well in the viscous BLs (Shishkina et al., 2010). When $\operatorname{Pr} \ll 1$, the thermal diffusion, represented by $\kappa$, is much larger than the momentum diffusion, represented by $\nu$, and therefore, the viscous BLs become extremely thin at large $R a$. Thus, there exist only a few DNS of thermal convection for a combination of large $R a$ and very low Prandtl numbers, $P r \leq$ 0.025, which, moreover, have been conducted exclusively for the RayleighBénard configuration (Schumacher et al., 2016; Scheel \& Schumacher, 2016, 2017; Horn \& Schmid, 2017; Vogt et al., 2018).

DNS of turbulent flows at extremely large $G r$ and extremely small Prandtl numbers, for example convective flow of magnesium that develops in a titanium reduction reactor (characterised by $G r$ of the order of $10^{12}$ ), is currently unrealisable. Therefore, further development of reduced mathematical models is still required. Computational codes for large-eddy simulations (LES) of turbulent thermal convection in liquid metals, verified against the corresponding experiments and DNS, can be useful in solving this kind of problem (Teimurazov et al., 2017).

Although knowledge on thermal convection in liquid metals is required for the development of safe and efficient liquid metal heat exchangers, the experimental database of the corresponding measurements remains quite restricted due to the known difficulties in conducting thermal measurements in liquid metals. Apart from general problems, occurring from the high temperature and aggressivity of liquid sodium, natural convective flows are known to be relatively slow and are very sensitive to the imposed disturbances. While probe measurements in the core part of pipe and channel flows are possible (Heinzel et al., 2017; Onea et al., 2017a,b), since the flow is influenced only downstream, they will unavoidably induce too strong disturbances in the case of natural thermal convection.

Thus, IC measurements in liquid sodium at large $R a$ and the corresponding precise numerical simulations are in great demand and, therefore, we devote our present work to this topic. Combining experiments, DNS and LES, we aim to paint a complementary picture of turbulent thermal liquid-sodium convection. Using these three viewpoints on liquid-sodium convection helps us to overcome 




Figure 4.1: Sketch of the experimental facility, which consists of: (1) a cylindrical convection cell, (2) a hot heat exchanger chamber, (3) a cold heat exchanger chamber, (4) a heated copper plate, (5) a cooled copper plate and (6) inductor coils. The convection cell (1) and heat exchangers $(2,3)$ are filled with liquid sodium. $D$ is the diameter and $L$ the length of the cylindrical convection cell (1), $\beta$ is the cell inclination angle, $\Theta$ the temperature drop between the hot and cold heat exchanger chambers, $\Delta$ the resulting temperature drop between the inner surfaces of the heated and cooled plates of the convection cell (1).

their individual difficulties and simultaneously verify their observations.

The outline of this work is as follows: in section 4.2 we introduce the experiment, DNS, LES and methods of data analysis. Section 4.3 presents the obtained results on how the global flow structures (mainly LSC) and their evolution in time affect the heat and momentum transport in case of liquidsodium convection. An analogy of the global flow structures and global heat transport in flows with similar values of $R a \operatorname{Pr}$ is also discussed there. The final section summarises our results.

\subsection{Methods}

\subsubsection{Experiment}

All experimental data presented in this paper are obtained at the experimental facility described in detail in Khalilov et al. (2018). The convection cell is made of a stainless steel pipe with a $3.5 \mathrm{~mm}$ thick wall. The inner length of the convection cell is $L=216 \mathrm{~mm}$ and the inner diameter $D=212 \mathrm{~mm}$. 


\begin{tabular}{ccc} 
& $\begin{array}{c}\lambda \\
(\mathrm{W} / \mathrm{mK})\end{array}$ & $\begin{array}{c}\kappa \times 10^{6} \\
\left(\mathrm{~m}^{2} / \mathrm{s}\right)\end{array}$ \\
\hline Stainless steel & 17 & 4.53 \\
$\mathrm{Na}$ & 84.6 & 66.5 \\
$\mathrm{Cu}$ & 391.5 & 111 \\
\hline
\end{tabular}

Table 4.1: The thermal conductivity $\lambda$ and the thermal diffusivity $\kappa$ of stainless steel, liquid sodium $(\mathrm{Na})$ and copper $(\mathrm{Cu})$ at the mean temperature of the experiment of about $410 \mathrm{~K}$.

Both end faces of the convection cell are separated from the heat exchanger chambers by $1 \mathrm{~mm}$ thick copper discs, see a sketch in figure 4.1. The convection cell is filled with liquid sodium. The heat exchanger chambers are filled also with liquid sodium and the temperature there is kept constant. The thin end-face copper plates are intensively washed with liquid sodium from the chamber sides, ensuring homogeneous temperature distributions at their surfaces (Kolesnichenko et al., 2017). The entire set-up is placed on a swing frame, so that the convection cell can be tilted from a vertical position to a horizontal one. Inclination of the convection cell is then characterised by the angle $\beta$ between the vertical and the cylinder axis, see figure 4.1.

Obviously, the boundary conditions in a real liquid-metal experiment and the idealised boundary conditions that are considered in numerical simulations, are different. For example, in the simulations, the cylindrical sidewall is assumed to be adiabatic, while in the real experiment it is made from $3.5 \mathrm{~mm}$ thick stainless steel and is additionally covered by a $30 \mathrm{~mm}$ thick layer of mineral wool. In table 4.1, the thermal characteristics of stainless steel, liquid sodium and copper are presented. One can see that the thermal diffusivity of the stainless steel, although being smaller compared to that of liquid sodium, is not negligible. Copper, which is known to be the best material for the heat exchangers in the experiments with moderate or high $\operatorname{Pr}$ fluids, has the thermal diffusivity of the same order as the thermal diffusivity of sodium. Therefore, massive copper plates would not provide a uniform temperature at the surfaces of the plates (Kolesnichenko et al., 2015). To avoid this undesirable inhomogeneity, in our experiment, instead of thick copper plates, we use rather thin ones, which are intensively washed from the outside by liquid sodium of prescribed temperature (see figure 4.1). The latter process takes place in two heat exchanger chambers, a hot one and a cold one, which are equipped with induction coils. A sodium flow in each heat exchanger chamber is provided by a travelling magnetic field. The induction coils are attached 
near the outer end faces of the corresponding heat exchanger, thus ensuring that the electromagnetic influence of the inductors on the liquid metal in the convection cell is negligible (Kolesnichenko et al., 2017). Typical velocities of the sodium flows in the heat exchangers are approximately $1 \mathrm{~m} / \mathrm{s}$, being an order of magnitude higher than the convective velocity inside the convection cell.

In all conducted experiments, the mean temperature of liquid sodium inside the convection cell is approximately $T_{\mathrm{m}}=139.8^{\circ} \mathrm{C}$, for which the Prandtl number equals $\operatorname{Pr} \approx 0.0093$. Each experiment is performed for a prescribed and known applied temperature difference $\Theta=T_{\text {hot }}-T_{\text {cold }}$, where $T_{\text {hot }}$ and $T_{\text {cold }}$ are the time-averaged temperatures of sodium in, respectively, the hot and cold heat exchanger chambers, which are measured close to the copper plates (see figure 4.1).

In any hot liquid-metal experiment, there exist unavoidable heat losses due to the high temperature of the set-up. To estimate the corresponding power losses $Q_{\text {loss }}$, one needs to measure additionally the power, which is required to maintain the same mean temperature $T_{\mathrm{m}}$ of liquid sodium inside the convection cell, but under the condition of equal temperatures in both heat exchanger chambers, that is, for $\Theta=0$. From $Q_{\text {loss }}$ and the total power consumption $Q$ in the convective experiment at $\Theta \neq 0$, one calculates the effective power $Q_{\text {eff }}=Q-Q_{\text {loss }}$ in any particular experiment.

Although the thermal resistance of the two thin copper plates themselves is negligible, the sodium-copper interfaces provide additional effective thermal resistance of the plates mainly due to inevitable oxide films. The temperature drop $\Delta_{\mathrm{pl}}$ through both copper plates covered by the oxide films could be calculated then from $\Delta_{\mathrm{pl}}=Q_{\mathrm{eff}} R_{\mathrm{pl}}$, as soon as the effective thermal resistance of the plates, $R_{\mathrm{pl}}$, is known. Note that for a fixed mean temperature $T_{\mathrm{m}}$, the value of $R_{\mathrm{pl}}$ depends on $\Theta$, since the effective thermal conductivities of the two plates are different due to their different temperatures.

The values of $R_{\mathrm{pl}}(\Theta)$ for different $\Theta$ are calculated in a series of auxiliary measurements for the case of $\beta=0$ and stable temperature stratification, where the heat is applied from above, to suppress convection. In this purely conductive case, the effective thermal resistance of the plates, $R_{\mathrm{pl}}$, can be calculated from $\Theta$ and the measured effective power $\bar{Q}_{\text {eff }}$ from the relation $\Theta=\bar{Q}_{\text {eff }}\left(R_{\mathrm{Na}}+R_{\mathrm{pl}}\right)$, where the thermal resistance of the liquid sodium equals $R_{\mathrm{Na}}=L /\left(\lambda_{\mathrm{Na}} S\right)$ with $\lambda_{\mathrm{Na}}$ being the liquid-sodium thermal conductivity and $S=\pi R^{2}$ with the cylinder radius $R$.

Using the above measured effective thermal resistances of the plates, $R_{\mathrm{pl}}(\Theta)$, 
in any convection experiment for a given $R a$ and $\beta$ and the mean temperature $T_{\mathrm{m}}$, the previously unknown temperature drop $\Delta$ inside the convection cell can be calculated from $\Theta, R_{\mathrm{pl}}(\Theta)$ and measured $Q_{\text {eff }}$ as follows:

$$
\Delta=\Theta-\Delta_{\mathrm{pl}}, \quad \Delta_{\mathrm{pl}}=Q_{\mathrm{eff}} R_{\mathrm{pl}} .
$$

Note that in all our measurements in liquid sodium, for all considered inclination angles of the convection cell, the obtained mean temperature and the temperature drop within the cell equal, respectively, $T_{\mathrm{m}} \approx 139.8^{\circ}$ and $\Delta \approx 25.3 \mathrm{~K}$.

The Nusselt number is then calculated as

$$
N u=\frac{L Q_{\mathrm{eff}}}{\lambda_{\mathrm{Na}} S \Delta}
$$

For a comparison of the experimental results with the DNS and LES results, where the temperatures at the plates inside the convection cell are known $a$ priori, the experimental temperature at the hot plate, $T_{+}$, and that at the cooled plate, $T_{-}$, are calculated as follows:

$$
T_{+}=T_{\mathrm{m}}+\Delta / 2, \quad T_{-}=T_{\mathrm{m}}-\Delta / 2 .
$$

The Rayleigh number in the experiment is evaluated as

$$
R a \equiv \alpha g \Delta D^{4} /(L \kappa \nu)
$$

which slightly differs from the value defined in (4.1), since $D$ is slightly smaller than $L$. Thus, for $\alpha=2.56 \times 10^{-4} \mathrm{~K}^{-1}, g=9.81 \mathrm{~m} / \mathrm{s}^{2}, \Delta=25.3 \mathrm{~K}, \nu=$ $6.174 \times 10^{-7} \mathrm{~m}^{2} / \mathrm{s}$ and $\kappa=6.651 \times 10^{-5} \mathrm{~m}^{2} / \mathrm{s}$, the Rayleigh number equals $R a=(1.42 \pm 0.03) \times 10^{7}$.

For a deep analysis of the convective liquid-sodium flows, the convection cell is equipped with 28 thermocouples, each with an isolated junction of $1 \mathrm{~mm}$. The thermocouples are located on 8 lines aligned parallel to the cylinder axis, see figure 4.2. The azimuthal locations of these lines are distributed with an equal azimuthal step of $45^{\circ}$ and are marked in figure 4.2 by capital letters $\mathrm{A}$ to $\mathrm{H}$ (counterclockwise, if looking from the cold end face). The line $\mathrm{A}$ has the upper position if $\beta>0^{\circ}$. On each of the 8 lines (A to $\mathrm{H}$ ), 3 or 5 thermocouples are placed. Thus, all thermocouples are located in five cross-sections of the convection cell, which are parallel to the end faces. The thermocouples are installed inside the convection cell at the same distance of $17 \mathrm{~mm}$ 

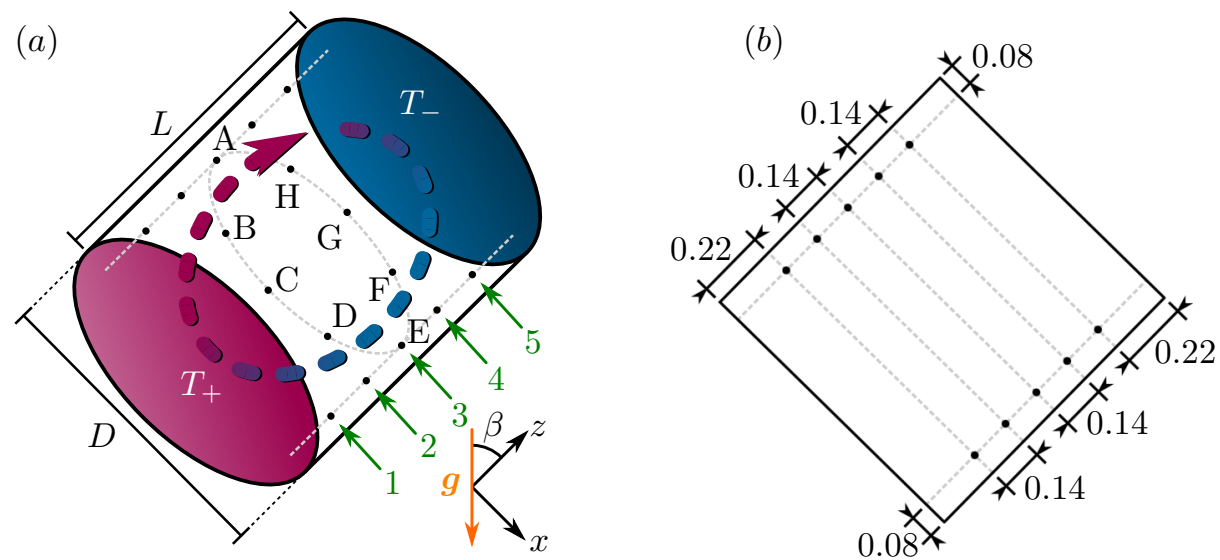

Figure 4.2: (a) Sketch of an inclined convection cell. $D$ is the diameter and $L$ the height of the cylindrical sample, $\beta$ is the inclination angle, $T_{+}$ $\left(T_{-}\right)$the temperature of the heated (cooled) surfaces. Positioning and naming of the 40 probes inside the cylinder, as considered in the DNS (all combinations of the azimuthal locations A, B, C, D, $\mathrm{E}, \mathrm{F}, \mathrm{G}, \mathrm{H}$ and circles $1, \ldots, 5)$ and 28 probes in the experiments (all combinations of the eight locations $\mathrm{A}, \ldots, \mathrm{H}$ for the circles 1, 3 and 5 plus four additional probes: A2, A4, E2, E4). Note that the azimuthal locations are shown only for the circle 3 , not to overload the sketch. For any inclination angle $\beta>0^{\circ}$, the upper azimuthal location is A. (b) Sketch of a central vertical cross-section of the setup from figure $(a)$, with shown distances (normalised with $L=D$ ) between the neighbouring probes and between the probes and the side wall of the cylindrical convection cell. 
from the inner cylinder sidewall and thus are located on 5 circles, which are marked in figure 4.2 with numbers $1, \ldots, 5$. The circles 1,3 and 5 include eight thermocouples, and the circles 2 and 4 only two thermocouples (A and E).

In this paper, besides the measurements by Khalilov et al. (2018), where a single Rayleigh number $R a=(1.42 \pm 0.03) \times 10^{7}$ was considered, we present and analyse also new experimental data, which are obtained for a certain range of the Rayleigh number, based on different imposed temperature gradients.

\subsubsection{Direct numerical simulations}

The problem of inclined thermal convection within the Oberbeck-Boussinesq (OB) approximation, which is studied in the DNS, is defined by the following Navier-Stokes, temperature and continuity equations in cylindrical coordinates $(r, \phi, z)$ :

$$
\begin{aligned}
D_{t} \mathbf{u} & =\nu \boldsymbol{\nabla}^{2} \mathbf{u}-\boldsymbol{\nabla} p+\alpha g\left(T-T_{0}\right) \hat{\mathbf{e}} \\
D_{t} T & =\kappa \boldsymbol{\nabla}^{2} T, \\
\boldsymbol{\nabla} \cdot \mathbf{u} & =0,
\end{aligned}
$$

where $D_{t}$ denotes the substantial derivative, $\mathbf{u}=\left(u_{r}, u_{\phi}, u_{z}\right)$ the velocity vector field, with the component $u_{z}$ in the direction $z$, which is orthogonal to the plates, $p$ is the reduced kinetic pressure, $T$ the temperature, $T_{0}=\left(T_{+}+T_{-}\right) / 2$ and $\hat{\mathbf{e}}$ is the unit vector, $\hat{\mathbf{e}}=(-\sin (\beta) \cos (\phi), \sin (\beta) \sin (\phi), \cos (\beta))$. Within the considered OB approximation, it is assumed that the fluid properties are independent of the temperature and pressure, apart from the buoyancy term in the Navier-Stokes equation, where the density is taken linearly dependent on the temperature.

These equations are non-dimensionalised by using the cylinder radius $R$, the free-fall velocity $U_{f}$, the free-fall time $t_{f}$,

$$
U_{f} \equiv(\alpha g R \Delta)^{1 / 2}, \quad t_{f}=R(\alpha g R \Delta)^{-1 / 2}
$$

and the temperature drop between the heated plate and the cooled plate, $\Delta$, as units of length, velocity, time and temperature, respectively.

To close the system (4.7)-(4.9), the following boundary conditions are considered: no slip for the velocity at all boundaries, $\mathbf{u}=0$, constant temperatures $\left(T_{-}\right.$or $\left.T_{+}\right)$at the face ends of the cylinder and adiabatic boundary condition at the sidewall, $\partial T / \partial r=0$.

The resulting dimensionless equations are solved numerically with the finite- 
volume computational code GoLDFISH, which uses high-order interpolation schemes in space and a direct solver for the pressure (Kooij et al., 2018). No turbulence model is applied in the simulations. The utilised staggered computational grids of approximately $1.5 \times 10^{8}$ nodes, which are clustered near all rigid walls, are sufficiently fine to resolve the Kolmogorov microscales, see table 4.2. Statistical averaging is usually carried out for several hundred free-fall time units, which is sufficient not only for integral quantities like the Nusselt number and Reynolds number to converge but also for the flow fields. The exceptions are the most expensive DNS for liquid sodium. Statistical averaging in our liquid-sodium DNS is, however, the longest known for such low Prandtl numbers and the integral quantities and also the velocity and temperature profiles are converged to a reasonable degree.

Since simulations on such fine meshes are extremely expensive, only 4 inclination angles are considered for the main case of $\operatorname{Pr}=0.0094$ and $R a=$ $1.67 \times 10^{7}$, which are $\beta=0^{\circ}, \beta=36^{\circ}, \beta=72^{\circ}$ and $\beta=90^{\circ}$. To study similarities of the flows with respect to the global heat transport and global flow structures for an almost constant Grashof number, $G r \equiv R a / P r$, and for a fixed value of $R a P r$, some additional DNS of IC were conducted for the combinations of $\operatorname{Pr}=0.094$ with $R a=1.67 \times 10^{6}$ and $\operatorname{Pr}=1$ with $R a=10^{9}$. Note that the Grashof number of the auxiliary DNS $\left(G r=10^{9}\right)$ is slightly different from that of the liquid-sodium DNS $\left(G r=1.78 \times 10^{9}\right)$. In the former additional DNS, eight different inclination angles are considered, while in the latter additional DNS, 11 different values of $\beta$ are examined, see table 4.2. The computational grids, used in the auxiliary DNS for similar $R a P r$, contain only 3 nodes within each viscous boundary layer, however, a convergence study, using twice as many nodes in each direction, for 3 different inclination angles demonstrated a deviation in $N u$ and $R a$ within $1 \%$.

\subsubsection{Large-eddy Simulations}

At any fixed time slice, LES generally require more computational effort per computational node, than the DNS. However, since the LES are relieved from the requirement to resolve the spatial Kolmogorov microscales, one can use significantly coarser meshes in the LES compared to those in the DNS, as soon as the LES are verified against the measurements from the physical point of view and against the DNS from the numerical point of view. Thus, the verified LES open the possibility of obtaining reliable data faster, compared to the DNS, using modest computational resources.

In our study, the OB equations (4.7)-(4.9) of thermogravitational convection 


\begin{tabular}{|c|c|c|c|c|c|c|c|c|c|c|c|}
\hline & $R a$ & $\operatorname{Pr}$ & $\beta$ & $t_{\mathrm{avg}} / t_{f}$ & $N_{r}$ & $N_{\phi}$ & $N_{z}$ & $\mathcal{N}_{\text {th }}$ & $\mathcal{N}_{\mathrm{V}}$ & $\delta_{u} / L$ & $\delta_{\mathrm{th}} / L$ \\
\hline \multirow[t]{4}{*}{ DNS } & $1.67 \times 10^{7}$ & 0.0094 & $0^{\circ}$ & 157.2 & 384 & 512 & 768 & 82 & 7 & $3.4 \times 10^{-3}$ & $5.2 \times 10^{-2}$ \\
\hline & & & $36^{\circ}$ & 89.0 & 384 & 512 & 768 & 67 & 7 & $3.2 \times 10^{-3}$ & $4.1 \times 10^{-2}$ \\
\hline & & & $72^{\circ}$ & 72.0 & 384 & 512 & 768 & 69 & 7 & $3.2 \times 10^{-3}$ & $4.2 \times 10^{-2}$ \\
\hline & & & $90^{\circ}$ & 66.8 & 384 & 512 & 768 & 77 & 7 & $3.4 \times 10^{-3}$ & $4.8 \times 10^{-2}$ \\
\hline \multirow[t]{10}{*}{ LES } & $1.5 \times 10^{7}$ & 0.0093 & $0^{\circ}$ & 460.5 & 100 & 160 & 200 & 32 & 3 & $3.3 \times 10^{-3}$ & $5.4 \times 10^{-2}$ \\
\hline & & & $10^{\circ}$ & 629.4 & 100 & 160 & 200 & 31 & 3 & $3.2 \times 10^{-3}$ & $5.2 \times 10^{-2}$ \\
\hline & & & $20^{\circ}$ & 806.0 & 100 & 160 & 200 & 29 & 3 & $3.1 \times 10^{-3}$ & $4.9 \times 10^{-2}$ \\
\hline & & & $30^{\circ}$ & 468.2 & 100 & 160 & 200 & 27 & 3 & $3.1 \times 10^{-3}$ & $4.4 \times 10^{-2}$ \\
\hline & & & $40^{\circ}$ & 468.2 & 100 & 160 & 200 & 27 & 3 & $3.1 \times 10^{-3}$ & $4.3 \times 10^{-2}$ \\
\hline & & & $50^{\circ}$ & 928.8 & 100 & 160 & 200 & 26 & 3 & $3.1 \times 10^{-3}$ & $4.2 \times 10^{-2}$ \\
\hline & & & $60^{\circ}$ & 560.3 & 100 & 160 & 200 & 27 & 3 & $3.1 \times 10^{-3}$ & $4.2 \times 10^{-2}$ \\
\hline & & & $70^{\circ}$ & 460.5 & 100 & 160 & 200 & 27 & 3 & $3.1 \times 10^{-3}$ & $4.3 \times 10^{-2}$ \\
\hline & & & $80^{\circ}$ & 537.3 & 100 & 160 & 200 & 28 & 3 & $3.2 \times 10^{-3}$ & $4.6 \times 10^{-2}$ \\
\hline & & & $90^{\circ}$ & 652.4 & 100 & 160 & 200 & 30 & 3 & $3.3 \times 10^{-3}$ & $5.0 \times 10^{-2}$ \\
\hline \multirow[t]{8}{*}{ DNS } & $1.67 \times 10^{6}$ & 0.0940 & $0^{\circ}$ & 4000 & 95 & 128 & 192 & 13 & 3 & $1.21 \times 10^{-2}$ & $6.1 \times 10^{-2}$ \\
\hline & & & $9^{\circ}$ & 1000 & 95 & 128 & 192 & 12 & 3 & $1.24 \times 10^{-2}$ & $5.7 \times 10^{-2}$ \\
\hline & & & $18^{\circ}$ & 2000 & 95 & 128 & 192 & 12 & 3 & $1.19 \times 10^{-2}$ & $5.4 \times 10^{-2}$ \\
\hline & & & $27^{\circ}$ & 1000 & 95 & 128 & 192 & 11 & 3 & $1.15 \times 10^{-2}$ & $5.2 \times 10^{-2}$ \\
\hline & & & $36^{\circ}$ & 2000 & 95 & 128 & 192 & 11 & 3 & $1.13 \times 10^{-2}$ & $5.1 \times 10^{-2}$ \\
\hline & & & $54^{\circ}$ & 2000 & 95 & 128 & 192 & 11 & 3 & $1.15 \times 10^{-2}$ & $5.0 \times 10^{-2}$ \\
\hline & & & $72^{\circ}$ & 1000 & 95 & 128 & 192 & 11 & 3 & $1.11 \times 10^{-2}$ & $5.2 \times 10^{-2}$ \\
\hline & & & $90^{\circ}$ & 1000 & 95 & 128 & 192 & 13 & 3 & $1.11 \times 10^{-2}$ & $5.9 \times 10^{-2}$ \\
\hline \multirow[t]{11}{*}{ DNS } & $10^{9}$ & 1 & $0^{\circ}$ & 420 & 384 & 512 & 768 & 16 & 11 & $5.2 \times 10^{-3}$ & $7.8 \times 10^{-3}$ \\
\hline & & & $9^{\circ}$ & 385 & 384 & 512 & 768 & 15 & 10 & $5.0 \times 10^{-3}$ & $7.7 \times 10^{-3}$ \\
\hline & & & $18^{\circ}$ & 403 & 384 & 512 & 768 & 15 & 10 & $4.6 \times 10^{-3}$ & $7.6 \times 10^{-3}$ \\
\hline & & & $27^{\circ}$ & 465 & 384 & 512 & 768 & 15 & 9 & $4.3 \times 10^{-3}$ & $7.6 \times 10^{-3}$ \\
\hline & & & $36^{\circ}$ & 447 & 384 & 512 & 768 & 15 & 8 & $3.9 \times 10^{-3}$ & $7.6 \times 10^{-3}$ \\
\hline & & & $45^{\circ}$ & 356 & 384 & 512 & 768 & 15 & 7 & $3.6 \times 10^{-3}$ & $7.5 \times 10^{-3}$ \\
\hline & & & $54^{\circ}$ & 366 & 384 & 512 & 768 & 15 & 7 & $3.2 \times 10^{-3}$ & $7.4 \times 10^{-3}$ \\
\hline & & & $63^{\circ}$ & 382 & 384 & 512 & 768 & 15 & 6 & $2.9 \times 10^{-3}$ & $7.7 \times 10^{-3}$ \\
\hline & & & $72^{\circ}$ & 448 & 384 & 512 & 768 & 16 & 6 & $2.8 \times 10^{-3}$ & $8.0 \times 10^{-3}$ \\
\hline & & & $81^{\circ}$ & 182 & 384 & 512 & 768 & 15 & 6 & $2.7 \times 10^{-3}$ & $8.4 \times 10^{-3}$ \\
\hline & & & $90^{\circ}$ & 36 & 384 & 512 & 768 & 17 & 6 & $2.6 \times 10^{-3}$ & $8.7 \times 10^{-3}$ \\
\hline
\end{tabular}

Table 4.2: Details on the conducted DNS and LES, including the time of statistical averaging, $t_{\text {avg }}$, normalised with the free-fall time $t_{f}$; number of nodes $N_{r}, N_{\phi}, N_{z}$ in the directions $r, \phi$ and $z$, respectively; the number of the nodes within the thermal boundary layer, $\mathcal{N}_{\text {th }}$, and within the viscous boundary layer, $\mathcal{N}_{\mathrm{v}}$, and the relative thickness of the viscous boundary layer $\delta_{u} / L(4.19)$ and the thermal boundary layer $\delta_{\mathrm{th}} / L(4.18)$. 
with the LES approach for small-scale turbulence modelling are solved numerically using the open-source package OpenFOAM 4.1 (Weller et al., 1998) for $\operatorname{Pr}=0.0093$ and $R a=1.5 \times 10^{7}$ and 10 different inclination angles, equidistantly distributed between $\beta=0^{\circ}$ and $\beta=90^{\circ}$.

The package is configured as follows. The used LES model is that by Smagorinsky-Lilly (Deardorff, 1970) with the Smagorinsky constant $C_{s}=0.17$, which is compatible with the value of the Kolmogorov spectrum constant for the inertial subrange. The turbulent Prandtl number in the core part of the domain equals $\operatorname{Pr}_{t}=0.9$ and smoothly vanishes close to the rigid walls. According to different models for liquid metals, the $\mathrm{Pr}_{t}$ value can be higher than this one (Chen et al., 2013). To estimate the effect of the $P r_{t}$ value on the simulation results we carried out additional LES with $\operatorname{Pr}_{t}$ values up to 4.12 and showed that the specific choice of $P r_{t}$ does not significantly affect the results. The utilised finite-volume solver is buoyantBoussinesqPimpleFoam with the pressure implicit with splitting of operators (PISO) algorithm by Issa (1986). Time integration is realised with the implicit Euler scheme; the diffusive and convective terms are also treated linearly (more precisely, using the filteredLinear scheme). The resulting systems of linear equations are solved with the preconditioned conjugate gradient method with the diagonal-based incomplete Cholesky preconditioner for the pressure and preconditioned biconjugate gradient method with the diagonal incomplete lower-upper (LU) preconditioner for other flow components (Fletcher, 1976; Ferziger \& Perić, 2002).

All simulations are carried out on a collocated non-equidistant computational grid consisting of 2.9 million nodes, see table 4.2. The grid has a higher density of nodes near the boundaries, in order to resolve the boundary layers. Further details on the numerical method, construction of the computational grid and model verification can be found in Mandrykin \& Teimurazov (2019).

\subsubsection{Methods of data analysis}

Nusselt number and Reynolds number. The main response characteristics of the convective system are the global heat and momentum transport represented by the dimensionless Nusselt number $N u$ and Reynolds number $R e$, respectively. Within the OB approximation, the Nusselt number equals

$$
N u=\left\langle\Omega_{z}\right\rangle_{\mathrm{z}}
$$


where $\Omega_{z}$ is a component of the heat-flux vector along the cylinder axis,

$$
\Omega_{z} \equiv \frac{u_{z} T-\kappa \partial_{z} T}{\kappa \Delta / L}
$$

and $\langle\cdot\rangle_{z}$ denotes the average in time and over a cross-section at any distance $z$ from the heated plate.

The Reynolds number can be defined in different ways and one of the common definitions is based on the total kinetic energy of the system,

$$
R e \equiv(L / \nu) \sqrt{\langle\mathbf{u} \cdot \mathbf{u}\rangle}
$$

Here $\langle\cdot\rangle$ denotes the averaging in time and over the entire volume. We consider also the large-scale Reynolds number

$$
R e_{\mathrm{U}} \equiv(L / \nu) \sqrt{\left\langle\langle\mathbf{u}\rangle_{\mathrm{t}}^{2}\right\rangle_{\mathrm{V}}}
$$

where $\langle\cdot\rangle_{\mathrm{t}}$ denotes the averaging in time and $\langle\cdot\rangle_{\mathrm{V}}$ the averaging over the whole convection cell. Following Teimurazov \& Frick (2017), we also evaluate the Reynolds number based on the volume-averaged velocity fluctuations, or smallscale Reynolds number, as

$$
R e_{u^{\prime}} \equiv(L / \nu) \sqrt{\left\langle\left(\mathbf{u}-\langle\mathbf{u}\rangle_{\mathrm{t}}\right)^{2}\right\rangle}
$$

Finally, one can calculate the Reynolds number based on the "wind of turbulence" as follows:

$$
R e_{\mathrm{w}}=(L / \nu) \max _{z} U_{\mathrm{w}}(z),
$$

where the velocity of the wind, which is parallel to the heated or cooled plates, can be estimated by

$$
U_{\mathrm{w}}(z)=\sqrt{\left\langle u_{\phi}^{2}+u_{r}^{2}\right\rangle_{z}},
$$

where $u_{\phi}$ and $u_{r}$ are the azimuthal and radial components of the velocity, respectively.

In the experiments, the Reynolds number is evaluated based on the average of the estimated axial velocities between the probes along the positions A and E. The velocities are estimated as written below.

Boundary-layer thicknesses. Close to the heated and cooled plates, thermal and viscous boundary layers develop. The thickness of the thermal boundary 
layer is calculated as

$$
\delta_{\mathrm{th}}=L /(2 N u)
$$

This is the standard way to define the thickness of the thermal boundary layer under the assumption of pure conductive heat transport within this layer, cf. Ahlers et al. (2009).

Using the slope method (cf. Zhou \& Xia (2010)), from the $U_{\mathrm{w}}(z)$ profile along the cylinder axis, see equation (4.17), the viscous boundary-layer thickness $\delta_{u}$ is defined as follows:

$$
\delta_{u} \equiv \max _{z}\left\{U_{\mathrm{w}}(z)\right\}\left(\left.\frac{d U_{\mathrm{w}}}{d z}\right|_{z=0}\right)^{-1} .
$$

The same criterion was used also for vertical convection before in $\mathrm{Ng}$ et al. (2015), and can also be adopted to the general case of IC.

Properties of the large-scale circulation. In the DNS and LES, the information on all flow components is available at every small finite volume associated with any grid node. In the experiments, all the information about the flow structures is obtained from the 28 temperature probes located as shown in figure 4.2 and discussed in section 4.2.1. The probes are placed in five different horizontal cross-sections of the cylindrical sample, which are parallel to the heated or cooled surfaces. To make a comparison between the DNS, LES and the experiment possible, we measure the temperature and monitor its temporal evolution at the same locations in all three approaches. The only difference is that in the DNS and LES there are 8 virtual probes in each cross-section, while in the experiment, there are 8 probes in the cross-sections 1, 3 and 5 and only 2 probes in the cross-sections 2 and 4 . The azimuthal locations A to $\mathrm{H}$ in the experiment, DNS and LES are exactly the same.

From the temperature measurements at the above discussed locations, one can evaluate the phase and the strength of the so-called wind of turbulence, or large-scale circulation (LSC). To do so, the method by Cioni et al. (1997) is applied, which is widely used in RBC experiments (Brown \& Ahlers, 2006; Xi \& Xia, 2007; He et al., 2016; Bai et al., 2016; Khalilov et al., 2018) and simulations (Mishra et al., 2011; Stevens et al., 2011; Wagner et al., 2012; Ching et al., 2017). Thus, the temperature measured at 8 locations in the central cross-section, from A3 to $\mathrm{H} 3$ along the central circle 3 , is fitted by the cosine function

$$
T(\theta)=T_{\mathrm{m}}+\delta_{3} \cos \left(\theta-\theta_{3}\right)
$$




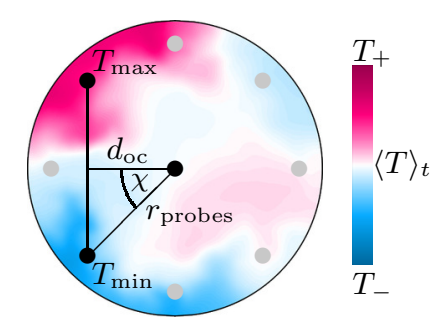

Figure 4.3: Sketch to illustrate the off-centre distance $d_{\mathrm{oc}}$ of the sloshing mode, as obtained by the temperature extrema extraction (TEE) method (cf. (4.21)), see Xi et al. (2009).

to obtain the orientation of the LSC, represented by the phase $\theta_{3}$, and the strength of the first temperature mode, i.e. the amplitude $\delta_{3}$, which indicates the temperature drop between the opposite sides of the cylinder sidewall. At the warmer part of the sidewall, the LSC carries the warm plumes from the heated plate towards the cold plate and on the opposite colder part, it carries the cold plumes in the opposite direction.

In a similar way one can evaluate the LSC phases $\theta_{1}$ and $\theta_{5}$ and the strengths $\delta_{1}$ and $\delta_{5}$ at other heights from the heated plates, i.e. along circle 1 (closer to the heated plate) and along circle 5 (closer to the cooled plate), respectively.

To extract the sloshing mode we use the method by Xi et al. (2009). The socalled temperature extrema extraction (TEE) method is executed as follows: First, the maximum and minimum of the eight probes at a certain time and horizontal level are determined and then a second-order polynomial is fitted exactly through that maximum/minimum using one neighbouring probe to each side ( 3 points in total per fit). The off-centre distance of the sloshing mode is defined as

$$
d_{\mathrm{oc}}=r_{\text {probes }} \cos (\chi),
$$

and it measures the shortest distance between the connecting line (of maximum and minimum temperature) and the centreline of the cylinder (cf. figure 4.3).

Velocity estimates. While in the DNS and LES the spatial distributions of all velocity components are available, direct measurements of the velocity in experiments on natural thermal convection in liquid sodium remain impossible so far. In order to estimate the velocities from the temperature measurements in the experiment, the cross-correlations for all combinations of any two neighbouring probes along the azimuthal locations from $\mathrm{A}$ to $\mathrm{H}$ are used.

For example, the normalised cross-correlation function $\left.C\right|_{A 1, A 2}(\tau)$ for the temporal dependences of the temperatures $\left.T\right|_{A 1}$ and $\left.T\right|_{A 2}$ measured by the 
probes at the locations A1 and A2 is calculated as follows:

$$
\left.C\right|_{A 1, A 2}(\tau) \propto \sum_{j}\left(\left.T\right|_{A 1}\left(t_{j}\right)-\left\langle\left. T\right|_{A 1}\right\rangle_{t}\right) \cdot\left(\left.T\right|_{A 2}\left(t_{j}+\tau\right)-\left\langle\left. T\right|_{A 2}\right\rangle_{t}\right) .
$$

The first maximum of the function $\left.C\right|_{A 1, A 2}(\tau)$ at $\tau=\tau_{c}$ provides the correlation time $\tau_{c}$. From the known distance between the probes A1 and A2 and the estimated time, $\tau_{c}$, which is needed for the flow to bring a thermal plume from the location $\mathrm{A} 1$ to $\mathrm{A} 2$, one can estimate the mean velocity of the flow between the locations $\mathrm{A} 1$ and $\mathrm{A} 2$.

In a similar way one estimates the mean velocities between the probes A2 and A3, etc., along the azimuthal location A. The mean velocities along the other azimuthal locations, from $\mathrm{B}$ to $\mathrm{H}$, are calculated analogously.

\subsection{Results and discussion}

In this section, we directly compare the results for inclined convection in a cylindrical container with a diameter-to-height aspect ratio of 1 , as they were obtained in the liquid-sodium DNS for $R a=1.67 \times 10^{7}$ and $\operatorname{Pr}=0.0094$, LES for $R a=1.5 \times 10^{7}$ and $\operatorname{Pr}=0.0093$ and liquid-sodium experiments for $R a=1.42 \times 10^{7}(\operatorname{Pr} \approx 0.0093)$, see tables 4.3 and 4.4 .

Further liquid-sodium experiments were conducted to measure the scaling relations of the Nusselt number versus the Rayleigh number in the RBC (the inclination angle $\left.\beta=0^{\circ}\right)$ and $\mathrm{VC}$ configurations $\left(\beta=90^{\circ}\right)$, for the $R a$-range around $R a=10^{7}$.

Additionally, we make a comparison with the auxiliary DNS results for $R a=$ $1.67 \times 10^{6}$ and $\operatorname{Pr}=0.094$, where the product of the Rayleigh number and Prandtl number, $R a \operatorname{Pr} \approx 1.57 \times 10^{5}$, is the same as in the main DNS for liquid sodium with $R a=1.67 \times 10^{7}$ and $\operatorname{Pr}=0.0094$, see tables 4.3 and 4.4. This auxiliary case is interesting for the following reasons. The ratio of the thermal diffusion time scale, $t_{\kappa}=R^{2} / \kappa$, to the free-fall time scale, $t_{f}=\sqrt{R / \alpha g \Delta}$ is in both cases the same, since $t_{\kappa} / t_{f} \sim \sqrt{R a P r}$. In contrast, in this auxiliary DNS case, the ratio $t_{\nu} / t_{f}$ of the viscous diffusion time scale $t_{\nu}=R^{2} / \nu$ to the free-fall time scale $t_{f}$ is approximately tenfold smaller than that in the main liquidsodium DNS, albeit being approximately tenfold larger than $t_{\kappa} / t_{f}$. Hence, thermal diffusion dominates over viscous diffusion in both considered sets of parameters and for similar $\operatorname{Ra} \operatorname{Pr}$ one might expect similar global temperature distributions and quantitatively similar heat and momentum transport in IC. Note that in the liquid-sodium DNS, the diffusion times are $t_{\kappa} \approx 140 t_{f}$ and 


\begin{tabular}{|c|c|c|c|c|c|c|c|c|}
\hline & $R a$ & $\operatorname{Pr}$ & $\beta$ & $N u$ & $R a$ & $\operatorname{Pr}$ & $\beta$ & $N u$ \\
\hline \multirow[t]{14}{*}{ Exp. } & $5.27 \times 10^{6}$ & 0.0094 & $0^{\circ}$ & 4.97 & $1.42 \times 10^{7}$ & 0.0093 & $20^{\circ}$ & 6.51 \\
\hline & $6.43 \times 10^{6}$ & 0.0097 & $0^{\circ}$ & 5.18 & $1.42 \times 10^{7}$ & 0.0093 & $30^{\circ}$ & 7.02 \\
\hline & $9.32 \times 10^{6}$ & 0.0096 & $0^{\circ}$ & 5.53 & $1.42 \times 10^{7}$ & 0.0093 & $40^{\circ}$ & 7.07 \\
\hline & $1.12 \times 10^{7}$ & 0.0095 & $0^{\circ}$ & 5.75 & $1.42 \times 10^{7}$ & 0.0093 & $50^{\circ}$ & 7.15 \\
\hline & $1.18 \times 10^{7}$ & 0.0093 & $0^{\circ}$ & 5.84 & $1.42 \times 10^{7}$ & 0.0093 & $60^{\circ}$ & 7.23 \\
\hline & $1.28 \times 10^{7}$ & 0.0094 & $0^{\circ}$ & 5.91 & $1.42 \times 10^{7}$ & 0.0093 & $70^{\circ}$ & 7.30 \\
\hline & $1.42 \times 10^{7}$ & 0.0093 & $0^{\circ}$ & 6.04 & $1.42 \times 10^{7}$ & 0.0093 & $80^{\circ}$ & 7.13 \\
\hline & $1.43 \times 10^{7}$ & 0.0091 & $0^{\circ}$ & 6.09 & $6.52 \times 10^{6}$ & 0.0095 & $90^{\circ}$ & 5.87 \\
\hline & $1.55 \times 10^{7}$ & 0.0093 & $0^{\circ}$ & 6.18 & $8.91 \times 10^{6}$ & 0.0094 & $90^{\circ}$ & 6.19 \\
\hline & $1.80 \times 10^{7}$ & 0.0091 & $0^{\circ}$ & 6.39 & $1.11 \times 10^{7}$ & 0.0093 & $90^{\circ}$ & 6.53 \\
\hline & $2.06 \times 10^{7}$ & 0.0088 & $0^{\circ}$ & 6.79 & $1.32 \times 10^{7}$ & 0.0091 & $90^{\circ}$ & 6.77 \\
\hline & $2.18 \times 10^{7}$ & 0.0088 & $0^{\circ}$ & 6.55 & $1.42 \times 10^{7}$ & 0.0093 & $90^{\circ}$ & 6.84 \\
\hline & $2.37 \times 10^{7}$ & 0.0086 & $0^{\circ}$ & 6.92 & $1.60 \times 10^{7}$ & 0.0090 & $90^{\circ}$ & 7.07 \\
\hline & $1.42 \times 10^{7}$ & 0.0093 & $10^{\circ}$ & 6.17 & $1.88 \times 10^{7}$ & 0.0086 & $90^{\circ}$ & 7.47 \\
\hline \multirow[t]{2}{*}{ DNS } & $1.67 \times 10^{7}$ & 0.0094 & 0 & 9.59 & $1.67 \times 10^{7}$ & 0.0094 & $72^{\circ}$ & 11.89 \\
\hline & $1.67 \times 10^{7}$ & 0.0094 & $36^{\circ}$ & 12.24 & $1.67 \times 10^{7}$ & 0.0094 & $90^{\circ}$ & 10.37 \\
\hline \multirow[t]{5}{*}{ LES } & $1.5 \times 10^{7}$ & 0.0093 & $0^{\circ}$ & 9.27 & $1.5 \times 10^{7}$ & 0.0093 & $50^{\circ}$ & 11.95 \\
\hline & $1.5 \times 10^{7}$ & 0.0093 & $10^{\circ}$ & 9.65 & $1.5 \times 10^{7}$ & 0.0093 & $60^{\circ}$ & 11.80 \\
\hline & $1.5 \times 10^{7}$ & 0.0093 & $20^{\circ}$ & 10.28 & $1.5 \times 10^{7}$ & 0.0093 & $70^{\circ}$ & 11.61 \\
\hline & $1.5 \times 10^{7}$ & 0.0093 & $30^{\circ}$ & 11.41 & $1.5 \times 10^{7}$ & 0.0093 & $80^{\circ}$ & 10.97 \\
\hline & $1.5 \times 10^{7}$ & 0.0093 & $40^{\circ}$ & 11.71 & $1.5 \times 10^{7}$ & 0.0093 & $90^{\circ}$ & 10.06 \\
\hline \multirow[t]{4}{*}{ DNS } & $1.67 \times 10^{6}$ & 0.0940 & $0^{\circ}$ & 8.21 & $1.67 \times 10^{6}$ & 0.0940 & $36^{\circ}$ & 9.79 \\
\hline & $1.67 \times 10^{6}$ & 0.0940 & $9^{\circ}$ & 8.78 & $1.67 \times 10^{6}$ & 0.0940 & $54^{\circ}$ & 9.95 \\
\hline & $1.67 \times 10^{6}$ & 0.0940 & $18^{\circ}$ & 9.23 & $1.67 \times 10^{6}$ & 0.0940 & $72^{\circ}$ & 9.55 \\
\hline & $1.67 \times 10^{6}$ & 0.0940 & $27^{\circ}$ & 9.64 & $1.67 \times 10^{6}$ & 0.0940 & $90^{\circ}$ & 8.55 \\
\hline \multirow[t]{6}{*}{ DNS } & $10^{9}$ & 1 & $0^{\circ}$ & 63.74 & $10^{9}$ & 1 & $54^{\circ}$ & 67.24 \\
\hline & $10^{9}$ & 1 & $9^{\circ}$ & 64.58 & $10^{9}$ & 1 & $63^{\circ}$ & 64.78 \\
\hline & $10^{9}$ & 1 & $18^{\circ}$ & 65.81 & $10^{9}$ & 1 & $72^{\circ}$ & 62.53 \\
\hline & $10^{9}$ & 1 & $27^{\circ}$ & 65.58 & $10^{9}$ & 1 & $81^{\circ}$ & 59.60 \\
\hline & $10^{9}$ & 1 & $36^{\circ}$ & 66.05 & $10^{9}$ & 1 & $90^{\circ}$ & 57.52 \\
\hline & $10^{9}$ & 1 & $45^{\circ}$ & 67.15 & & & & \\
\hline
\end{tabular}

Table 4.3: Nusselt numbers, as they were obtained in the experiments, DNS and LES. The uncertainty of the experiments is about $3 \%$, of the DNS it is about $4 \%$ and of the LES it is below $1 \%$. 


\begin{tabular}{|c|c|c|c|c|c|c|}
\hline & $R a$ & $\operatorname{Pr}$ & $\beta$ & $R e$ & $R e_{u^{\prime}}$ & $R e_{U}$ \\
\hline DNS & $1.67 \times 10^{7}$ & 0.0094 & $\begin{array}{r}0^{\circ} \\
36^{\circ} \\
72^{\circ} \\
90^{\circ}\end{array}$ & $\begin{array}{l}1.80 \times 10^{4} \\
1.95 \times 10^{4} \\
1.34 \times 10^{4} \\
1.01 \times 10^{4}\end{array}$ & $\begin{array}{r}1.27 \times 10^{4} \\
1.02 \times 10^{4} \\
5.5 \times 10^{3} \\
4.3 \times 10^{3}\end{array}$ & $\begin{array}{r}1.27 \times 10^{4} \\
1.66 \times 10^{4} \\
1.22 \times 10^{4} \\
9.1 \times 10^{3}\end{array}$ \\
\hline LES & $1.5 \times 10^{7}$ & 0.0093 & $\begin{array}{l}0^{\circ} \\
10^{\circ} \\
20^{\circ} \\
30^{\circ} \\
40^{\circ} \\
50^{\circ} \\
60^{\circ} \\
70^{\circ} \\
80^{\circ} \\
90^{\circ}\end{array}$ & $\begin{array}{r}1.627 \times 10^{4} \\
1.702 \times 10^{4} \\
1.766 \times 10^{4} \\
1.772 \times 10^{4} \\
1.723 \times 10^{4} \\
1.676 \times 10^{4} \\
1.517 \times 10^{4} \\
1.311 \times 10^{4} \\
1.147 \times 10^{4} \\
9.60 \times 10^{3}\end{array}$ & $\begin{aligned} 1.136 & \times 10^{4} \\
1.145 & \times 10^{4} \\
1.113 & \times 10^{4} \\
9.70 & \times 10^{3} \\
8.24 & \times 10^{3} \\
7.11 & \times 10^{3} \\
6.02 & \times 10^{3} \\
5.32 & \times 10^{3} \\
4.60 & \times 10^{3} \\
3.85 & \times 10^{3}\end{aligned}$ & $\begin{aligned} 1.165 & \times 10^{4} \\
1.258 & \times 10^{4} \\
1.371 & \times 10^{4} \\
1.483 & \times 10^{4} \\
1.513 & \times 10^{4} \\
1.518 & \times 10^{4} \\
1.392 & \times 10^{4} \\
1.199 & \times 10^{4} \\
1.051 & \times 10^{4} \\
8.80 & \times 10^{3}\end{aligned}$ \\
\hline DNS & $1.67 \times 10^{6}$ & 0.0940 & $\begin{array}{r}0^{\circ} \\
9^{\circ} \\
18^{\circ} \\
27^{\circ} \\
36^{\circ} \\
54^{\circ} \\
72^{\circ} \\
90^{\circ}\end{array}$ & $\begin{array}{r}1.33 \times 10^{3} \\
1.42 \times 10^{3} \\
1.46 \times 10^{3} \\
1.46 \times 10^{3} \\
1.43 \times 10^{3} \\
1.33 \times 10^{3} \\
9.9 \times 10^{2} \\
7.3 \times 10^{2}\end{array}$ & $\begin{array}{c}1.24 \times 10^{3} \\
1.10 \times 10^{3} \\
8.1 \times 10^{2} \\
7.0 \times 10^{2} \\
6.3 \times 10^{2} \\
3.7 \times 10^{2} \\
7.7 \times 10^{1} \\
0\end{array}$ & $\begin{array}{r}4.7 \times 10^{2} \\
1.10 \times 10^{3} \\
1.22 \times 10^{3} \\
1.28 \times 10^{3} \\
1.28 \times 10^{3} \\
1.28 \times 10^{3} \\
9.9 \times 10^{2} \\
7.3 \times 10^{2}\end{array}$ \\
\hline DNS & $10^{9}$ & 1 & $\begin{array}{r}0^{\circ} \\
9^{\circ} \\
18^{\circ} \\
27^{\circ} \\
36^{\circ} \\
45^{\circ} \\
54^{\circ} \\
63^{\circ} \\
72^{\circ} \\
81^{\circ} \\
90^{\circ}\end{array}$ & $\begin{aligned} 4.72 & \times 10^{3} \\
5.13 & \times 10^{3} \\
4.97 & \times 10^{3} \\
4.65 & \times 10^{3} \\
4.06 & \times 10^{3} \\
3.79 & \times 10^{3} \\
3.31 & \times 10^{3} \\
2.44 & \times 10^{3} \\
1.66 & \times 10^{3} \\
1.23 & \times 10^{3} \\
8.4 & \times 10^{2}\end{aligned}$ & $\begin{array}{r}4.25 \times 10^{3} \\
3.62 \times 10^{3} \\
2.50 \times 10^{3} \\
2.14 \times 10^{3} \\
1.91 \times 10^{3} \\
1.84 \times 10^{3} \\
1.68 \times 10^{3} \\
1.29 \times 10^{3} \\
7.5 \times 10^{2} \\
2.9 \times 10^{2} \\
1.2 \times 10^{1}\end{array}$ & $\begin{array}{r}2.05 \times 10^{3} \\
3.63 \times 10^{3} \\
4.29 \times 10^{3} \\
4.12 \times 10^{3} \\
3.59 \times 10^{3} \\
3.31 \times 10^{3} \\
2.85 \times 10^{3} \\
2.07 \times 10^{3} \\
1.48 \times 10^{3} \\
1.19 \times 10^{3} \\
8.4 \times 10^{2}\end{array}$ \\
\hline
\end{tabular}

Table 4.4: Reynolds numbers as they were obtained in the DNS and LES, see the definitions (4.13), (4.14) and (4.15). The numerical uncertainty of the Reynolds numbers was estimated to be about $3 \%$ for DNS and below $1 \%$ for LES. 
$t_{\nu} \approx 14900 t_{f}$.

Another set of auxiliary DNS of IC is conducted for $R a=10^{9}$ and $\operatorname{Pr}=1$ for comparison. In this case, the Grashof number, $G r \equiv R a / \operatorname{Pr}=10^{9}$, is similar to that in the main liquid-sodium case $\left(G r \approx 1.8 \times 10^{9}\right)$, but for this Prandtlnumber-one case and the liquid-sodium case we generally do not expect a close similarity of the global flow characteristics.

A summary of the conducted simulations and experiments can be found in table 4.2. The free-fall time in the experiments equals $t_{f}=\sqrt{R /(\alpha g \Delta)} \approx 1.3 \mathrm{~s}$ and is similar to that in the main DNS and LES. Thus, the conducted DNS cover only approximately two minutes of the real-time experiment, which was conducted for approximately 7 hours. One should note that although the DNS statistical averaging time is quite short, collecting approximately $100 t_{f}$ statistics for the case $\beta=0^{\circ}$ consumed approximately $390000 \mathrm{CPUh}$ at the SuperMUC at the Leibniz Supercomputing Centre and required approximately 60 days of runtime.

In the remaining part of this section we investigate integral time-averaged quantities such as the global heat transport (Nusselt number) and the global momentum transport (Reynolds number), provide the evidence of a quantitative agreement between the simulations and experiment and present a complementary picture of the dynamics of the large-scale flows in liquid-sodium IC.

\subsubsection{Time-averaged heat and momentum transport}

First, we examine the classical case of $\mathrm{RBC}$ without inclination $\left(\beta=0^{\circ}\right)$. The time-averaged mean heat fluxes, represented by the Nusselt numbers, are presented in figure 4.4 .

There is an excellent agreement of the DNS and LES data, for example, the Nusselt number deviates less than $1 \%$ and the Reynolds number around $5 \%$ for the RBC and VC cases. Also in figure 4.4 we compare our numerical results with the DNS by Scheel \& Schumacher (2017), for $\operatorname{Pr}=0.005$ and $\operatorname{Pr}=0.025$. Our numerical results for $\operatorname{Pr}=0.0094$ and $\operatorname{Pr}=0.0093$ take place between the cited results by Scheel \& Schumacher (2017), as expected. Note that our LES and DNS and the DNS by Scheel \& Schumacher (2017) were conducted using completely different codes (the Nek5000 spectral element package in the latter case), but nevertheless lead to consistent results. This verifies to the fact that the obtained results are independent of the numerical method.

The experimental data exhibit generally lower Nusselt numbers (approximately $35 \%$ ) compared to the numerical data and this can be explained by the following two reasons. First, the ideal boundary conditions of constant 




Figure 4.4: Nusselt number versus Rayleigh number, as obtained in the RBC experiments for different Rayleigh numbers, $\operatorname{Pr} \approx 0.009, \beta=0^{\circ}$ (open circles) with the effective scaling $N u \approx 0.177 R a^{0.215}$ (solid line); the RBC experiment for $R a=1.42 \times 10^{7}, \operatorname{Pr}=0.0093$, $\beta=0^{\circ}$ (filled circle; this run is the longest one $(7 \mathrm{~h})$ in the series of measurements. For the same $R a$ and $\operatorname{Pr}$, the Nusselt numbers were also measured for different $\beta$, see table 4.3); the $\mathrm{VC}$ experiments for different Rayleigh numbers, $\operatorname{Pr} \approx 0.009, \beta=90^{\circ}$ (open squares) with the effective scaling $N u \approx 0.178 R a^{0.222}$ (dash-dotted line); the inset shows the compensated Nusselt number for RBC and VC experimental data; the DNS for $R a=1.67 \times 10^{7}, \operatorname{Pr}=0.0094$, $\beta=0^{\circ}$ (filled diamond); the DNS for $R a=1.67 \times 10^{6}, \operatorname{Pr}=0.094$, $\beta=0^{\circ}$ (cross); the LES for $R a=1.5 \times 10^{7}, \operatorname{Pr}=0.0093, \beta=0^{\circ}$ (open triangle). Results of the RBC DNS by Scheel \& Schumacher (2017) for $\operatorname{Pr}=0.005$ (open diamonds) and for $\operatorname{Pr}=0.025$ (pluses) and predictions for $\operatorname{Pr}=0.0094$ of the Grossmann \& Lohse (2000, 2001) theory considered with the pre-factors from Stevens et al. (2013) (dash line) are presented for comparison. Everywhere a cylindrical convection cell of the aspect ratio 1 is considered. 
temperatures at the plates cannot be provided in the experiments, since each emission of a sufficiently strong thermal plume affects, at least for a short time, the local temperature at the plate, which results in a reduction of the averaged heat flux compared to that in the simulations with the ideal boundary conditions. Second, the impossibility of measuring the temperature directly at the outer surfaces of the copper plates leads to a slight overestimation of $\Theta$ and $\Delta$ (see figure 4.1) and, hence, of the effective Rayleigh numbers for the measured Nusselt numbers.

Scaling relations. For $\operatorname{Pr}=0.0094$, the predictions by the Grossmann \& Lohse $(2000,2001)$ theory, with the prefactors from Stevens et al. (2013), are shown in figure 4.4 between the obtained experimental and numerical data. The experimental data for a certain $R a$-range around $R a=10^{7}$, shown in figure 4.4 , follow a scaling relation

$$
N u \approx(0.16 \pm 0.01) R a^{0.22 \pm 0.06} \quad\left(\mathrm{RBC}, \quad \beta=0^{\circ}\right)
$$

The fitted scaling exponent $\gamma$ for the RBC case is smaller than the scaling exponents measured in liquid gallium $(\gamma=0.249$, King \& Aurnou (2013)) and liquid mercury $(\gamma=0.285$, Takeshita et al. (1996)). The Prandtl number for both fluids is $\operatorname{Pr} \approx 0.025$.

Horanyi et al. (1999) measured the heat transport in liquid sodium at $\operatorname{Pr} \approx 0.006$ for $R a \leq 4.5 \times 10^{6}$. They used larger aspect ratios from 4.5 to 20 , while their highest $R a$ measurements were conducted in cells with the smallest aspect ratio. Nevertheless, their $N u \approx 5$ at their highest $R a \approx 4.5 \times 10^{6}$ is similar to $N u=4.97$ at our lowest $R a=5.27 \times 10^{6}$. Furthermore, Horanyi et al. (1999) found that $N u$ scales as $R a^{0.25}$, which agrees with our results.

The GL theory predicts a local scaling exponent ranging from 0.23 $\left(R a=5 \times 10^{6}\right)$ to $0.26\left(R a=3 \times 10^{7}\right)$ for $\operatorname{Pr}=0.0094$. This results in a mean scaling exponent of approximately 0.25 for this interval of Rayleigh numbers.

We fit $\log (N u)$ versus $\log (R a)$ with a second-order polynomial to estimate the uncertainty of the scaling exponent in our experiments. The local scaling exponent of this fit ranges from $0.16\left(R a=5 \times 10^{6}\right)$ to $0.27\left(R a=3 \times 10^{7}\right)$, thus, we estimate the uncertainty of the scaling exponent to be $\Delta \gamma= \pm 0.06$. A large variation of the local scaling exponent can be seen in Glazier et al. (1999) for liquid mercury in an aspect ratio one cylinder. Their local scaling exponent for small Rayleigh numbers $\left(\sim 10^{6}-10^{8}\right)$ is 0.25 , while their average scaling exponent is 0.285. Glazier et al. (1999) argue that the "strong steady 

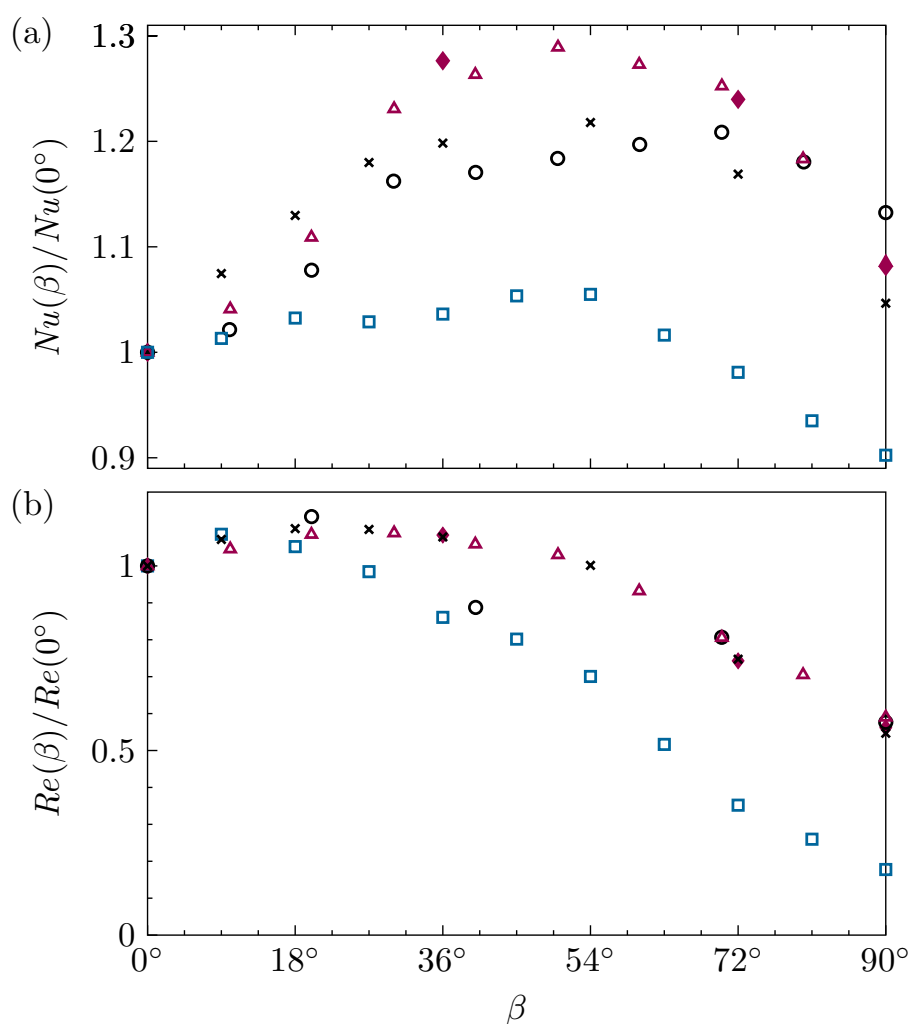

Figure 4.5: (a) Normalised Nusselt number $N u(\beta) / N u\left(0^{\circ}\right)$ versus the inclination angle $\beta$, as obtained in the DNS for $R a=1.67 \times 10^{7}$, $\operatorname{Pr}=0.0094$ (filled diamonds), the LES for $R a=1.5 \times 10^{7}$, $\operatorname{Pr}=0.0093$ (open triangles), the DNS for $R a=1.67 \times 10^{6}$, $\operatorname{Pr}=0.094$ (crosses), the experiments for $R a=1.42 \times 10^{7}$, $\operatorname{Pr} \approx 0.0093$ (open circles) and the DNS for $R a=10^{9}, \operatorname{Pr}=1$ (squares). (b) Normalised Reynolds number versus the inclination angle $\beta$, as obtained in the same DNS and LES as in $(a)$; similar symbols are used as in $(a)$.

bulk mean flow in the cell of aspect ratio one" reduces the scaling exponent. Presumably, we see a similar effect in our measurements.

In figure 4.4 we also present the measured scaling relations for $N u$ versus $R a$ for the case of $\mathrm{VC}\left(\beta=90^{\circ}\right)$. The scaling relation in $\mathrm{VC}$ is found to be quite similar to that in RBC, namely

$$
N u \approx(0.18 \pm 0.01) R a^{0.22 \pm 0.06} \quad\left(\mathrm{VC}, \quad \beta=90^{\circ}\right)
$$

The absolute values of the Nusselt numbers in VC are, however, larger than in $\mathrm{RBC}$.

Time-averaged heat transport in inclined convection. In figure $4.5 \mathrm{a}$, the Nusselt numbers in IC are presented, which are normalised by $N u$ of the RBC 
case, for the same $R a$ and $\operatorname{Pr}$, i.e. the dependence of $N u(\beta) / N u\left(0^{\circ}\right)$ on the inclination angle $\beta$. Very remarkable is that, for similar $R a$ and $P r$, the DNS and LES deliver very similar values of $N u$. As previously discussed for the limiting cases (RBC and VC), the deviation between DNS and LES data for the Nusselt numbers is only a few per cent. One can see that the relative increase $N u(\beta) / N u\left(0^{\circ}\right)$ of the numerical data is up to 10 percentage points higher than that of the experimental data for an inclination angle of approximately $50^{\circ}$. This is accompanied by a shift of the inclination angle with maximal heat transport from approximately $50^{\circ}$ in our numerical results to $70^{\circ}$ in our experimental results. On the one hand, one may take into account that the Rayleigh number in the experiment is slightly smaller $\left(R a=1.42 \times 10^{7}\right)$ compared to that in the DNS $\left(R a=1.67 \times 10^{7}\right)$ and $\operatorname{LES}\left(R a=1.5 \times 10^{7}\right)$. On the other hand, the heat transport in the inclined case is enhanced up to $29 \%$ which might reduce the heat loss $Q_{\text {loss }}$. However, $Q_{\text {loss }}$ is measured in the isothermal case, maintaining the mean temperature in the cylinder (cf. sec. 4.2.1). If the heat loss in the inclined case is less than in the isothermal reference case, then also the calculated effective power $Q_{\text {eff }}=Q-Q_{\text {loss }}$ is lower, and so is the Nusselt number.

As discussed above, we want to compare our results for liquid sodium with the DNS data for similar $R a P r$ and with the DNS data for similar $R a / P r$, as in our liquid-sodium measurements and numerical simulations. Figure 4.5a and table 4.3 show that the obtained Nusselt numbers for the same $R a P r$ $\left(R a=1.67 \times 10^{6}, \operatorname{Pr}=0.094\right)$ are in good agreement (the difference is less than 10 percentage points) with the liquid-sodium experimental results ( $R a=$ $\left.1.42 \times 10^{7}, \operatorname{Pr} \approx 0.0093\right)$. Remarkable is that, not only the relative Nusselt number, $N u(\beta) / N u\left(0^{\circ}\right)$, but also the absolute values of $N u$ are very similar in the liquid-sodium case and in the case of different $\operatorname{Pr}<1$ but the same $\mathrm{Ra} \mathrm{Pr}$. In contrast to that, the $\mathrm{Nu}$-dependence on the inclination angle in the case of the same Grashof number is different, as expected. In that case, the maximal relative increase of the Nusselt number due to the cell inclination is only approximately $6 \%$, while in the liquid-sodium case it is up to $29 \%$.

Time-averaged momentum transport in inclined convection. In figure $4.5 \mathrm{~b}$ and table 4.4, the results for the Reynolds number are presented for the liquidsodium measurements and simulations as well as for the auxiliary DNS. Again, the agreement between the experiments, DNS and LES for liquid sodium is excellent, usually less than $10 \%$ between DNS and LES. The dependences of $\operatorname{Re}(\beta) / \operatorname{Re}\left(0^{\circ}\right)$ on the inclination angle, obtained in the liquid-sodium DNS 


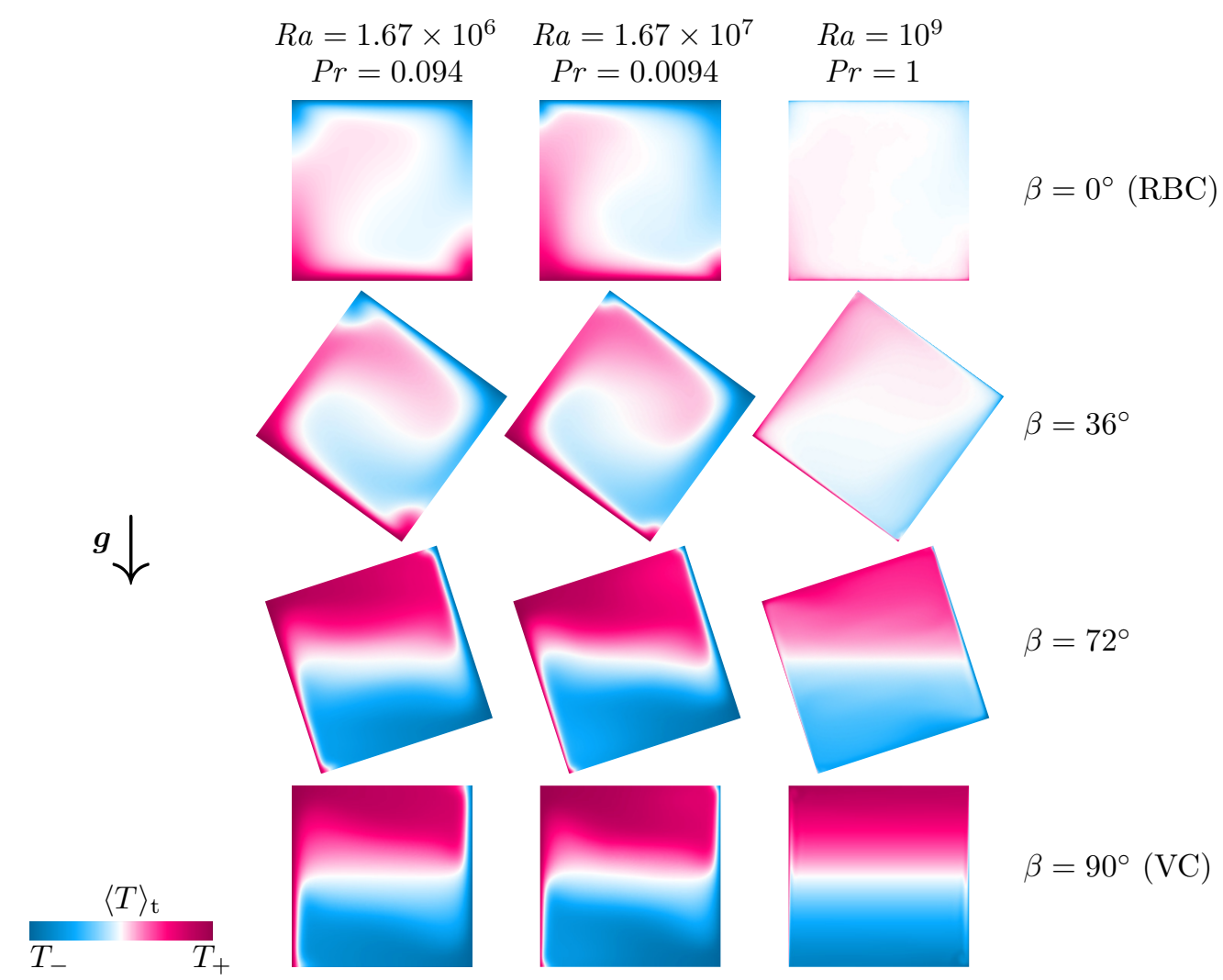

Figure 4.6: Table of vertical slices through the time-averaged temperature in the plane of the LSC, as obtained in the DNS for $R a=1.67 \times 10^{6}$, $\operatorname{Pr}=0.094$ (left column); $R a=1.67 \times 10^{7}, \operatorname{Pr}=0.0094$ (central column) and $R a=10^{9}, \operatorname{Pr}=1$ (right column). From top to bottom, the inclination angle $\beta$ changes from $\beta=0^{\circ}$ (RBC case) through $\beta=36^{\circ}$ and $\beta=72^{\circ}$ to $\beta=90^{\circ}$ (VC case).

and in the DNS for the same product of $R a P r$, demonstrate perfect agreement (less than two percentage points difference). The values of $\operatorname{Re}(\beta) / \operatorname{Re}\left(0^{\circ}\right)$ first slightly increase (around 10\%) with the inclination angle and then smoothly decrease, so that the Reynolds number $\operatorname{Re}\left(90^{\circ}\right)$ in the VC case is significantly smaller (only approximately $50 \%$ ) than the Reynolds number $\operatorname{Re}\left(0^{\circ}\right)$ in the $\mathrm{RBC}$ case. Here one should notice that the absolute values of $R e$ in the liquidsodium case are significantly larger than in the IC flows for a similar $\operatorname{Ra} \operatorname{Pr}$. In the case of the almost similar Grashof number, the Reynolds numbers decrease much faster with increasing inclination angle than in the liquid-sodium case.

Time-averaged flow structures in inclined convection. Since the Nusselt numbers and relative Reynolds numbers behave very similarly in the liquidsodium IC experiments and in the DNS for similar $R a P r$, we compare the time-averaged flow structures for these cases in figures 4.6 and 4.7. In these figures, the time-averaged temperature (figure 4.6) and the time-averaged com- 


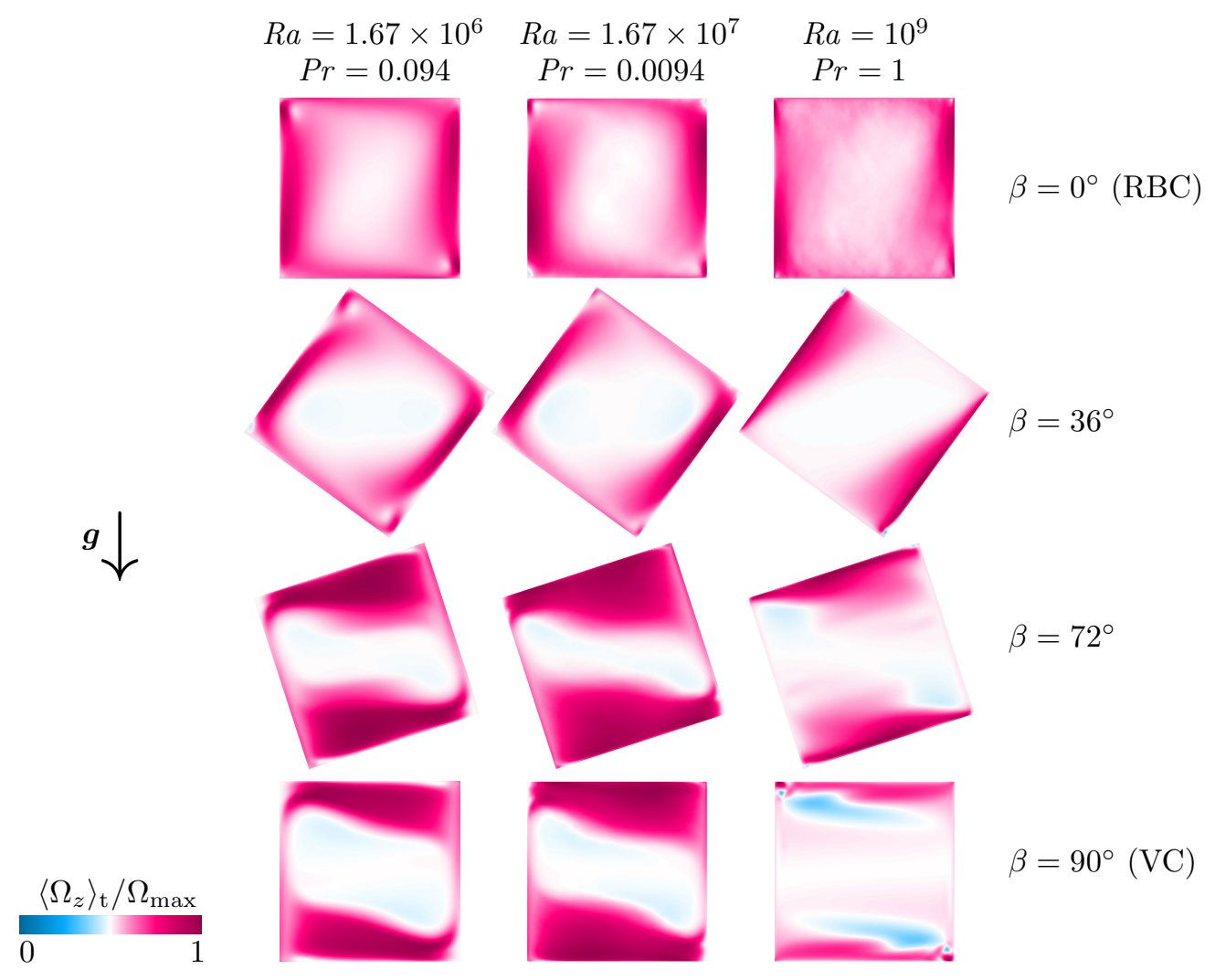

Figure 4.7: Table of vertical slices in the plane of the LSC of the time-averaged component of the heat flux vector parallel to the cylinder axis, $\left\langle\Omega_{z}\right\rangle_{\mathrm{t}}$, normalised by its maximal value through the entire volume, $\Omega_{\max }$, as obtained in the DNS for $R a=1.67 \times 10^{6}, \operatorname{Pr}=0.094$ (left column); $R a=1.67 \times 10^{7}, \operatorname{Pr}=0.0094$ (central column) and $R a=10^{9}, \operatorname{Pr}=1$ (right column). From top to bottom, the inclination angle $\beta$ changes from $\beta=0^{\circ}$ (RBC case) through $\beta=36^{\circ}$ and $\beta=72^{\circ}$ to $\beta=90^{\circ}$ (VC case). 
ponent of the heat-flux vector parallel to the cylinder axis $\left\langle\Omega_{z}\right\rangle_{\mathrm{t}}$ (figure 4.7 ) are presented in the plane of the LSC, for different inclination angles. One can see that both the temperature distributions and the heat-flux distributions, in the liquid-sodium case and in the case of a similar $R a P r$, look almost identically. In contrast to them, the corresponding distributions for a similar Grashof number look very different. The difference is especially pronounced for the inclination angle $\beta=36^{\circ}$. While in the liquid-sodium flow for $\beta=36^{\circ}$ there persist two intertwined plumes, a hot one and a cold one, the temperature in the Prandtl-number-one case is better mixed (figure 4.6) and the heat-flux distribution appears in a form of two triangular-shaped separated spots (figure 4.7).

From what is presented above, one can see that at almost similar Grashof numbers convection of a Prandtl-number-one fluid leads neither to similar integral quantities like $N u$ or $R e$, nor to similar heat flow structures in IC. Thus, it is clearly different to small-Prandtl-number IC flows. In contrast to that, the small-Prandtl-number IC flows of similar $\operatorname{Ra} \operatorname{Pr}$ have similar Nusselt numbers $N u$, similar relative Reynolds numbers $\operatorname{Re}(\beta) / \operatorname{Re}\left(0^{\circ}\right)$ and similar mean temperature and heat-flux distributions.

\subsubsection{Temperature and velocity profiles}

In this section we analyse the temperature and velocity profiles. The focus thereby is on the following two aspects. First, we compare the experimentally and numerically obtained profiles through the probes (positions A to $\mathrm{H}$ ) along the lines aligned parallel to the cylinder axes. Second, we compare the velocity profiles, obtained in the DNS and LES, with the velocities evaluated from the correlation times between two neighbouring probes in the experiment, in order to validate the method used in the experiment to estimate the Reynolds number.

In figure 4.8a, the time-averaged temperature profiles along the lines of the probe positions $\mathrm{A}$ to $\mathrm{H}$ are presented for the inclination angles $\beta=36^{\circ}$ (DNS) and $\beta=40^{\circ}$ (LES and experiments). Figure 4.8b shows analogous profiles for the inclination angles $\beta=72^{\circ}$ (DNS) and $\beta=70^{\circ}$ (LES and experiments). In both figures, the profiles at the positions $\mathrm{A}$ and $\mathrm{E}$ are presented, as well as the average of the profiles at the positions $\mathrm{B}$ and $\mathrm{H}$, the average of the profiles at the positions D and F and the average of the C-profile and G-profile. One can see that for the same locations, the LES and DNS profiles are almost indistinguishable, which again demonstrates quantitative agreement between the DNS and LES. The experimental data are available pointwise there, according 



Figure 4.8: Time-averaged temperature profiles at the positions $\mathrm{A}$ to $\mathrm{H}$ of the probes, as obtained in $(a)$ the DNS for $\beta=36^{\circ}$ and the LES and experiments for $\beta=40^{\circ}$ and in (b) the DNS for $\beta=72^{\circ}$ and the LES and experiments for $\beta=70^{\circ}$. Thick lines are the DNS data, thin lines are the LES data and symbols are the experimental data. The DNS data is for $R a=1.67 \times 10^{7}, \operatorname{Pr}=0.0094$, the LES data is for $R a=1.5 \times 10^{7}, \operatorname{Pr}=0.0093$ and the experiments are for $R a=1.42 \times 10^{7}, \operatorname{Pr} \approx 0.0093$. Data at the position A (pink solid lines, squares) and the position E (blue solid lines, circles); the average of the data at the positions $\mathrm{B}$ and $\mathrm{H}$ (pink dash-dotted lines, pentagons), the average of the data at the positions $\mathrm{D}$ and $\mathrm{F}$ (blue dash-dotted lines, triangles) and the average of the data at the positions $\mathrm{C}$ and $\mathrm{G}$ (black dotted and grey dash lines, diamonds). The data is averaged along opposite positions due to the expected symmetry and in order to reduce the number of lines in the plot. 

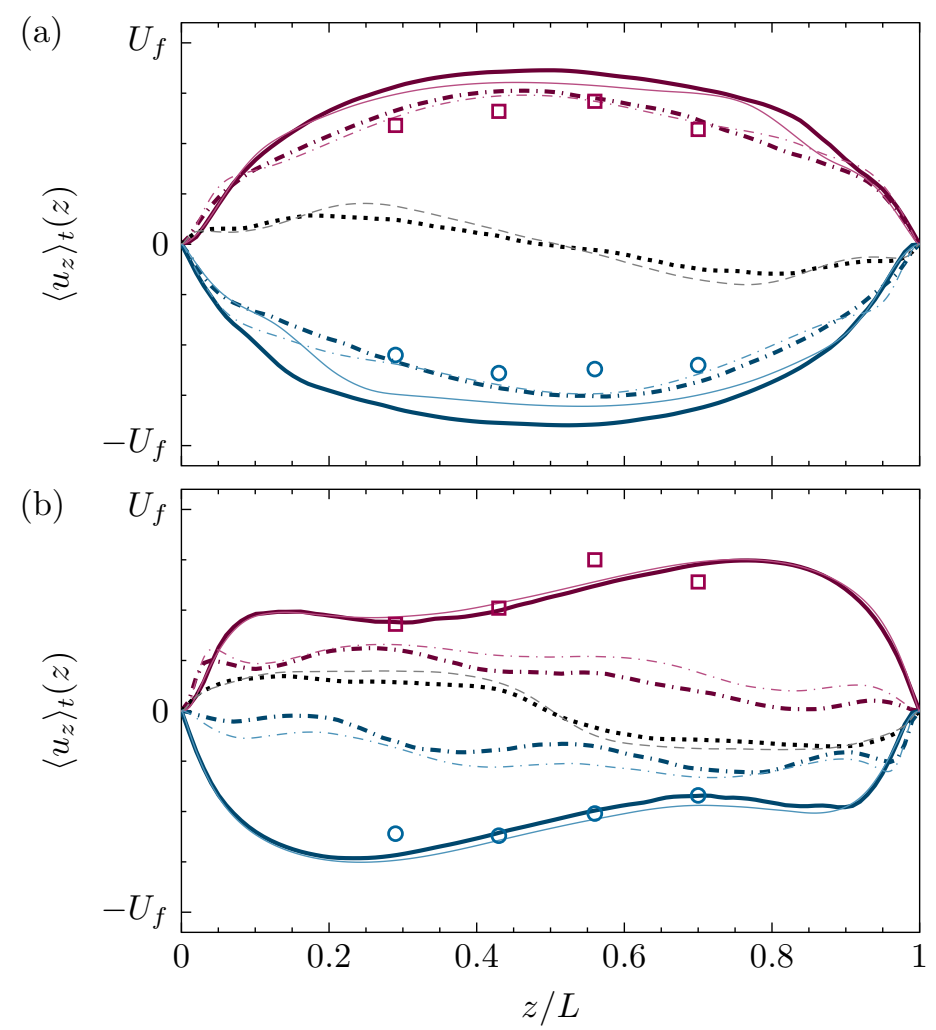

Figure 4.9: Time-averaged profiles of the velocity component $u_{z}$, which is parallel to the cylinder axis, considered at the positions $\mathrm{A}$ to $\mathrm{H}$ of the probes, as obtained in $(a)$ the DNS for $\beta=36^{\circ}$ and the LES and experiments for $\beta=40^{\circ}$ and in $(b)$ the DNS for $\beta=72^{\circ}$ and the LES and experiments for $\beta=70^{\circ}$. Thick lines are the DNS data, thin lines are the LES data and symbols are the experimental data. The DNS data is for $R a=1.67 \times 10^{7}, \operatorname{Pr}=0.0094$, the LES data is for $R a=1.5 \times 10^{7}, \operatorname{Pr}=0.0093$ and the experiments are for $R a=1.42 \times 10^{7}, \operatorname{Pr} \approx 0.0093$. Data at the position A (pink solid lines, squares) and the position E (blue solid lines, circles); the average of the data at the positions $\mathrm{B}$ and $\mathrm{H}$ (pink dash-dotted lines), the average of the data at the positions $\mathrm{D}$ and $\mathrm{F}$ (blue dash-dotted lines) and the average of the data at the positions $\mathrm{C}$ and $\mathrm{G}$ (black dotted and grey dash lines). The data is averaged along opposite positions due to the expected symmetry and in order to reduce the number of lines in the plot. 
to the 5 or 3 probes along each location, from A to $\mathrm{H}$. The measurements of the time-averaged temperatures are found to be in good agreement (largest deviation approximately $0.1 \Delta$ ) with the numerical data, taking into account that the Rayleigh number in the experiments is approximately $15 \%$ smaller than in the DNS.

At an inclination angle of approximately $\beta=36^{\circ}$ or $\beta=40^{\circ}$ (figure $4.8 \mathrm{a}$ ), the mean temperature gradient with respect to the direction $z$ across the plates is close to zero in the core part of the domain. This means that the turbulent mixing in this case is very efficient, which is also reflected in the increased Nusselt numbers that we studied before. In contrast to that, for the inclination angle approximately $\beta=70^{\circ}$ (figure $4.8 \mathrm{~b}$ ), the mean flow is stratified and the temperature profiles have non-vanishing gradients in the $z$-direction.

In figure 4.9, the time-averaged profiles of the velocity component $u_{z}$ along the line of the probe positions $(\mathrm{A}-\mathrm{H})$ parallel to the cylinder axis are presented for the same inclinations angles, as in figure 4.8. The velocity estimates at the locations between the neighbouring thermocouples, which are derived from the correlation times obtained in the temperature measurements, are found to be in agreement (largest deviation approximately $0.27 U_{f}$ ) with the DNS and LES data. Thus, this method to estimate the LSC velocity from the temperature measurement is proved to be a very reliable instrument in the IC liquid-sodium experiments.

The viscous boundary-layer thickness ((4.19) and table 4.2) for the liquidsodium DNS and LES agree within a small deviation of approximately $3 \%$. With inclination, the viscous boundary-layer thickness first decreases slightly (by a few per cent) and then gradually increases. Note that the viscous boundary layers defined on the velocity magnitude (Ching et al., 2017) and on the wall shear stress (Scheel \& Schumacher, 2016) are, respectively, approximately two and three times thicker than the boundary layer defined by (4.19) and table 4.2 .

\subsubsection{Dynamics of the large-scale flow}

In this section, we focus on the reconstruction of the rich structural dynamics of the large-scale IC flows in liquid sodium.

It is well known from the previous RBC studies that the LSC in RBC can show different azimuthal orientations (Brown \& Ahlers, 2006; Wagner et al., 2012) and can exhibit complicated dynamics with twisting (Funfschilling \& Ahlers, 2004; Funfschilling et al., 2008; He et al., 2016) and sloshing (Xi et al., 2006, 2009; Zhou et al., 2009; Brown \& Ahlers, 2009; Bai et al., 2016; Zürner 
et al., 2019). In very-low-Prandtl-number RBC, this complicated behaviour of the LSC was reported in experiments with mercury by Cioni et al. (1997) and in the simulations by Schumacher et al. (2016); Scheel \& Schumacher (2016, 2017).

In our simulations and experiments in liquid sodium, we observe the twisting and sloshing dynamics of the LSC in the RBC configuration of the flow, i.e. without any cell inclination, as well as for small inclination angles $\beta$ until a certain critical $\beta=\beta_{s}$. The experimental data suggest that a transition to the non-twisting behaviour of the LSC is quite sharp and it is presumably caused by the increasing stratification of the temperature at larger inclination angles (Khalilov et al., 2018).

No LSC cessations were captured in the experiments so far. LSC precession happens only in the RBC case (Khalilov et al., 2018). Only one LSC reversal was captured in a seven-hour-long RBC experiment in liquid sodium (Mamykin et al., 2018). Further investigations regarding LSC cessations and reversals need to be carried out in the future.

In figure 4.10, the time evolution of the volume-averaged components of the heat-flux vector parallel to the cylinder axis, $\left\langle\Omega_{z}\right\rangle_{\mathrm{V}}$ are presented, as they are obtained in the DNS for $R a=1.67 \times 10^{7}, \operatorname{Pr}=0.0094$ and four different inclination angles between $\beta=0^{\circ}$ (RBC) and $\beta=90^{\circ}$ (VC). Obviously, in the $\mathrm{RBC}$ case, the fluctuations of the heat flux around its mean value are extreme and reach up to $\pm 44 \%$ of $\left\langle\Omega_{z}\right\rangle$. The strength of the fluctuations gradually decreases with growing inclination angle $\beta$ and amount only to $\pm 3 \%$ of $\left\langle\Omega_{z}\right\rangle$ in the $\mathrm{VC}$ case. In figure 4.10 one can see that for the inclination angles $\beta=36^{\circ}$ and $\beta=72^{\circ}$, the mean heat transport is stronger than in the $\mathrm{RBC}$ or VC cases. This supports a general tendency that in small- $P r$ fluids the heat transport becomes more efficient, when the convection cell is tilted. Figure $4.5 \mathrm{a}$ and table 4.3 provide a more detailed evidence of this fact, based on our measurements and numerical simulations.

The twisting mode. In figure 4.11 we present the dynamics of the LSC twisting mode. There, the temporal evolution of the phases of the LSC in circle 1 (closer to the heated plate) and in circle 5 (closer to the cold plate) are presented for different inclinations angles $\beta$ of the convection cell filled with liquid sodium, as obtained in our DNS and measurements.

The main evidence for the existence of the twisting mode is the visible strong anti-correlation of the phases $\theta_{1}(t)$ and $\theta_{5}(t)$, which are measured via the probes at the circles 1 and 5 , respectively. It is present in the RBC case (figure 4.11a,b) 


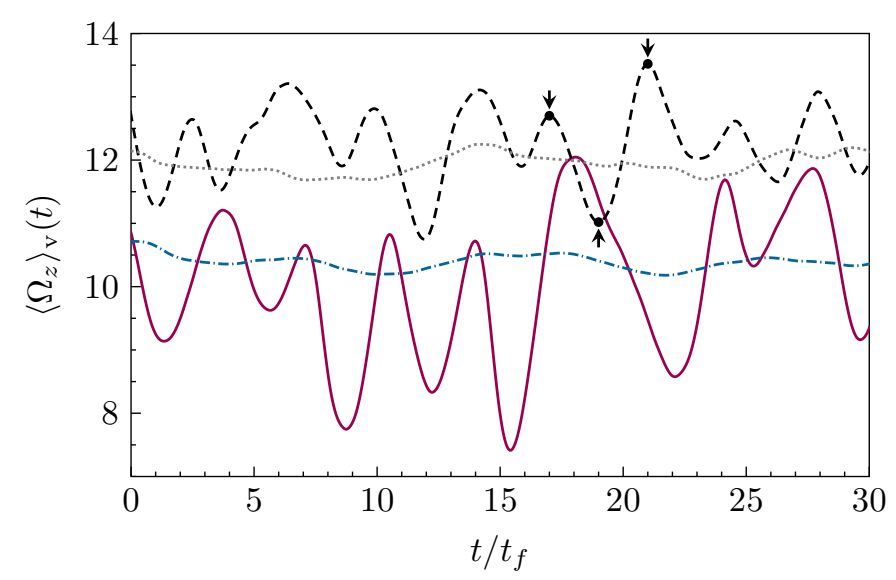

Figure 4.10: Time dependences of the volume-averaged component of the heat flux vector along the cylinder axis, $\left\langle\Omega_{z}\right\rangle_{\mathrm{V}}$, as obtained in the DNS for $R a=1.67 \times 10^{7}, \operatorname{Pr}=0.0094$ and four different inclination angles $\beta=0^{\circ}$ (solid line), $\beta=36^{\circ}$ (dash line), $\beta=72^{\circ}$ (dotted line) and $\beta=90^{\circ}$ (dash-dotted line). Time is normalised with $t_{f}=R(\alpha g R \Delta)^{-1 / 2}$. The arrows indicate the dimensionless times, which are marked in figure 4.12 with vertical lines and corresponding 3-dimensional snapshots are shown in figure 4.13.

and for the inclination angles $\beta=20^{\circ}$ (figure 4.11c) and $\beta=36^{\circ}$ (figure 4.11d). The measurements and DNS at the inclination angles $\beta \geq 40^{\circ}$ (figure 4.11e) show that, with increasing $\beta$, the above anti-correlation vanishes. At large inclination angles, there is no visible anti-correlation of the phases $\theta_{1}(t)$ and $\theta_{5}(t)$ and one can conclude that the twisting movement of the LSC is not present any more (figure 4.11f,g,h).

The dynamics of the twisting mode of the LSC can be further studied with the Fourier analysis. Thus, from the DNS data $\left(R a=1.67 \times 10^{7}, \operatorname{Pr}=\right.$ $0.0094)$ we obtain that the period duration $T_{s}(\beta)$ equals $T_{s}\left(0^{\circ}\right)=8.8 t_{f}$ for the $\mathrm{RBC}$ case and is equal to $T_{s}\left(36^{\circ}\right)=7.4 t_{f}$ for the inclination angle $\beta=36^{\circ}$. The experimental data give $T_{s}\left(0^{\circ}\right) \approx 9.2 t_{f}$ for $\mathrm{RBC}$ and $T_{s}\left(20^{\circ}\right) \approx 8.7 t_{f}$ for $\beta=20^{\circ}$. Note that the frequency $\omega$ of the LSC twisting is approximately proportional to the Reynolds number $\omega \cdot t_{\kappa} \sim R e$ (Cioni et al., 1997). Therefore, the slightly larger period durations in the experiments compared to those in the DNS are consistent with slightly lower Reynolds numbers and Rayleigh numbers there.

We also compare the twisting frequencies obtained in our DNS with results by Schumacher et al. (2016), Xi et al. (2009), Xie et al. (2013), Funfschilling \& Ahlers (2004). For low Prandtl numbers the numerical results by Schumacher et al. (2016) give a period of $4.8 t_{f}$ for $\operatorname{Pr}=0.021$ and $R a=10^{7}$. At higher Prandtl numbers the period is much longer. For water it is approximately 

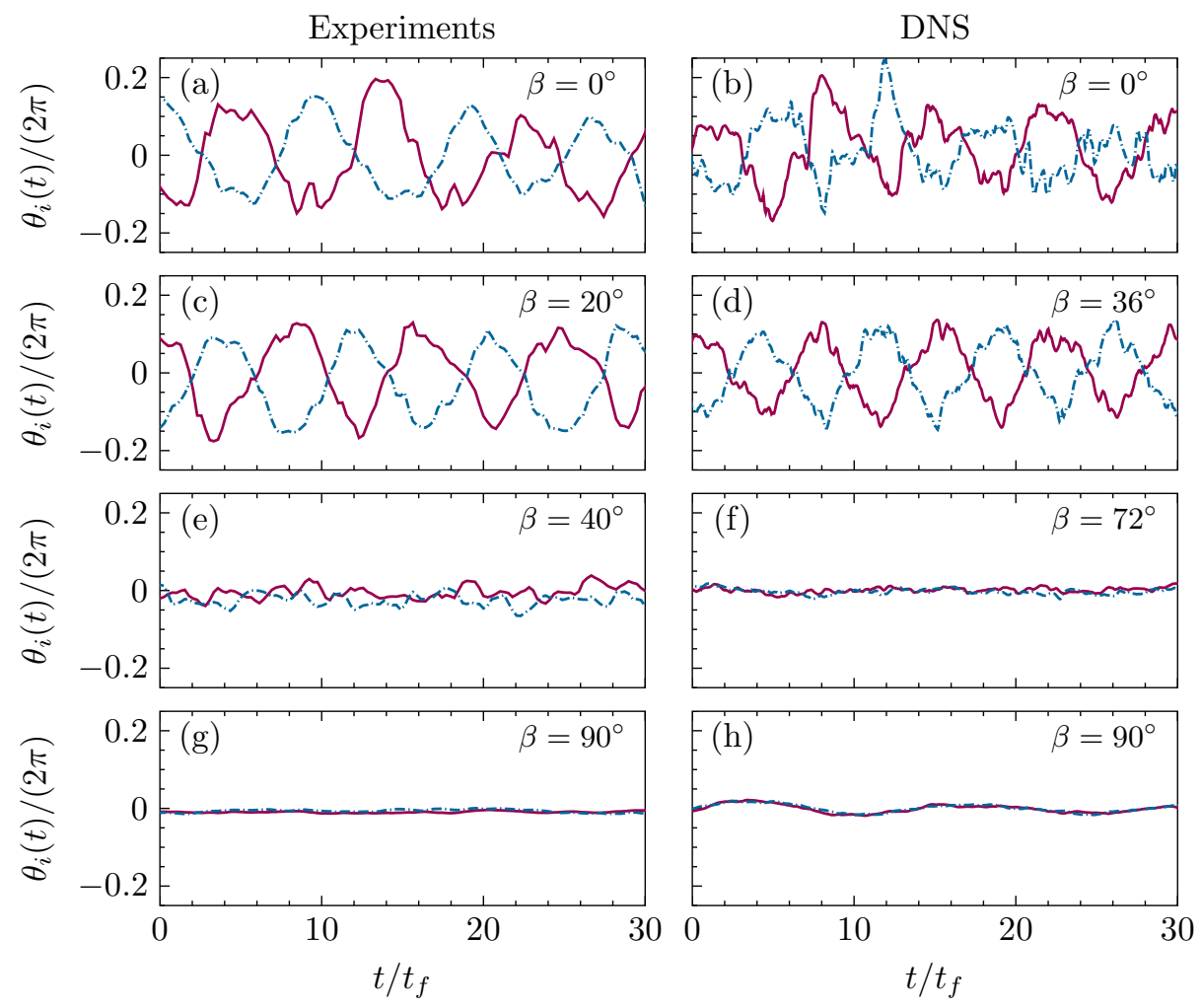

Figure 4.11: Temporal evolution of the phase $\theta_{1}$ in the circle 1 (solid lines) and of the phase $\theta_{5}$ in the circle 5 (dash-dotted line) of the convection cell (see locations of the circles in figure 4.2), as obtained in the experiments for $R a=1.42 \times 10^{7}, \operatorname{Pr} \approx 0.0093(a, c, e, g)$ and in the DNS for $R a=1.67 \times 10^{7}, \operatorname{Pr}=0.0094(b, d, f, h)$ for the cell inclination angles $\beta=0^{\circ}(a, b), \beta=20^{\circ}(c), \beta=36^{\circ}(d), \beta=40^{\circ}$ $(e), \beta=72^{\circ}(f)$ and $\beta=90^{\circ}(g, h)$. 
$30 t_{f}$ at $\operatorname{Pr}=5.3$ and $R a=5 \times 10^{9}$ (Xi et al., 2009). For even lager Prandtl number, $\operatorname{Pr}=19.4$, Xie et al. (2013) found $T_{s} \approx 85 t_{f}$ at $R a=2 \times 10^{11}$. The twisting frequency is lower with increasing $\operatorname{Pr}$ at high Prandtl numbers $(\operatorname{Pr}>1)$. However, the twisting frequency is slightly lower at $\operatorname{Pr}=0.0094$ compared to $\operatorname{Pr}=0.021$ for similar Rayleigh number.

The sloshing mode. Besides the twisting mode there is another mode present in the flow. This is the sloshing mode; it brings together the hot and cold streams of the LSC. Both the twisting and the sloshing mode are observed simultaneously, and the full LSC dynamics is determined by a combination of both modes. To quantify the sloshing motion, we calculate the off-centre distance (4.21) and plot it over time (fig. 4.12c) for the example case (DNS, $\left.R a=1.67 \times 10^{7}, \operatorname{Pr}=0.0094, \beta=36^{\circ}\right)$. The sloshing mode has the same frequency as the twisting mode (correlation of both signals is $>0.8$ ), but it shows a phase difference of approximately $\frac{1}{4}$-period. From the comparison of $d_{\mathrm{oc}}(t)$ and $\left\langle\Omega_{z}\right\rangle_{\mathrm{V}}(t)$ we learn that, first the hot and cold streams are brought close to each other, and then, with a short delay, the heat transport increases.

The sloshing mode is coherent through the entire height of the cylinder, quantified by a correlation of at least 0.8 between the sloshing signal $\left(d_{\text {oc }}\right)$ at mid-height circle 3 and the lower/higher circles (1, 2, 4 and 5). The correlation of circles 1 and 5 is approximately 0.75 .

Let us investigate the IC flow in liquid sodium for the inclination angle $\beta=36^{\circ}$, as in figure $4.11 \mathrm{~d}$, where a very strong LSC twisting is observed. In figure 4.12 we analyse this flow in more detail. Figure 4.12a presents the evolution of the temperature in time, which is measured by the probes B3, D3, F3 and H3 that are placed in the central circle 3.

One can see that the temperature dependencies on time at the locations B3 and F3 are synchronous. So are the temperature dependencies on time at the locations H3 and D3. The temperatures at the locations B3 and D3 are anticorrelated. So are the temperatures at the locations H3 and F3. Thus, when at the location B3 the fluid is extremely hot, the lowest temperature is obtained near D3, which is located only $90^{\circ}$ azimuthally below B3. Analogously, when the fluid is hot at the location H3, its lowest temperature is obtained near the location F3, which is $90^{\circ}$ below H3 (see also figure 4.13). These events happen at the times $t / t_{f}=17$ and $t / t_{f}=21$ in figure 4.12 , respectively. Thus, at the times $t / t_{f}=17$ and $t / t_{f}=21$ a big hot and a big cold plume approach each other very closely. This sloshing movement happens periodically, alternately, near one side of the sidewall, then on the opposite side. 
(a)

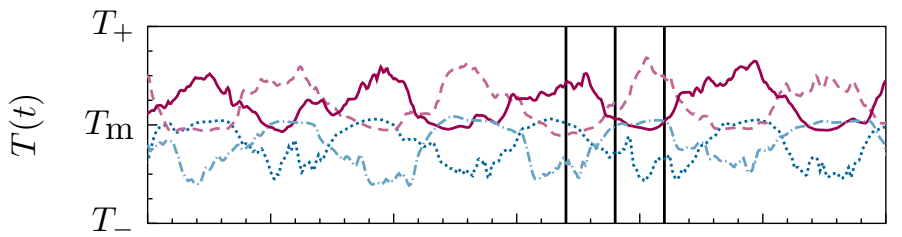

(b)

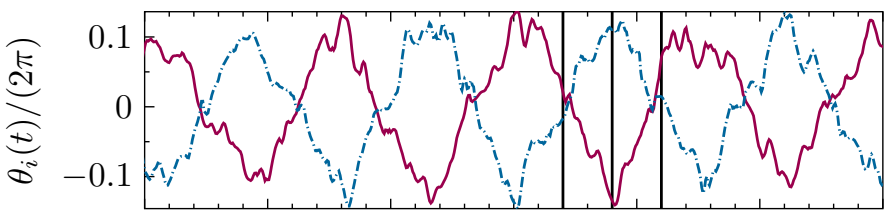

(c)

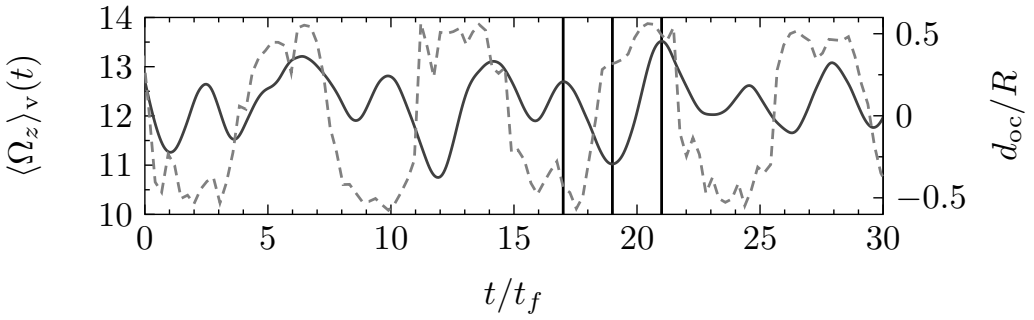

Figure 4.12: Temporal evolution of different quantities obtained in the DNS for $R a=1.67 \times 10^{7}, \operatorname{Pr}=0.0094$ and the cell inclination angle $\beta=36^{\circ}$ : (a) the temperature $T$ at the probes B3 (solid line), D3 (dash-dotted line), F3 (dotted line) and H3 (dash line); (b) the phase $\theta_{1}$ in the circle 1 (solid line) and the phase $\theta_{5}$ in the circle 5 (dash-dotted line) and $(c)$ the volume-averaged component of the heat flux vector along the cylinder axis, $\left\langle\Omega_{z}\right\rangle_{\mathrm{V}}$ (solid line) and the off-center-distance, $d_{\mathrm{oc}}$ (dashed line). The three vertical lines mark the times at which the snapshots in figure 4.13 are taken. The phases $\theta_{1}(t)$ and $\theta_{5}(t)$ in $(b)$ have a period of $T_{\theta}=$ $(7.4 \pm 0.2) t_{f}$, which is determined by the Fourier analysis. 

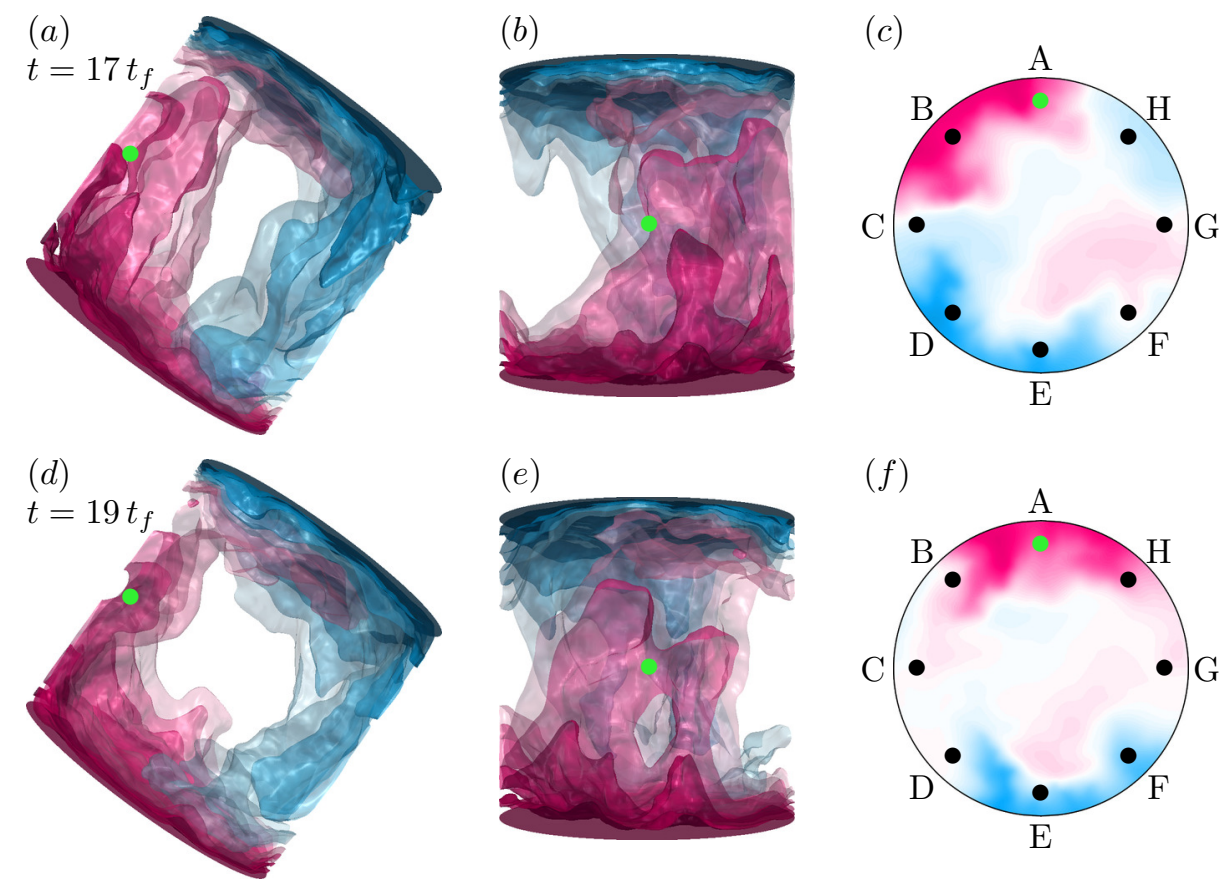

(e)

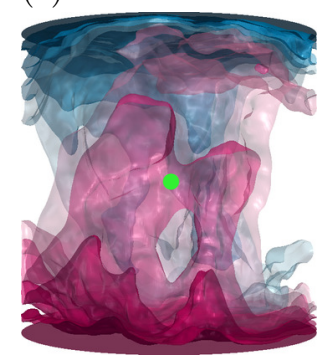

$(f)$
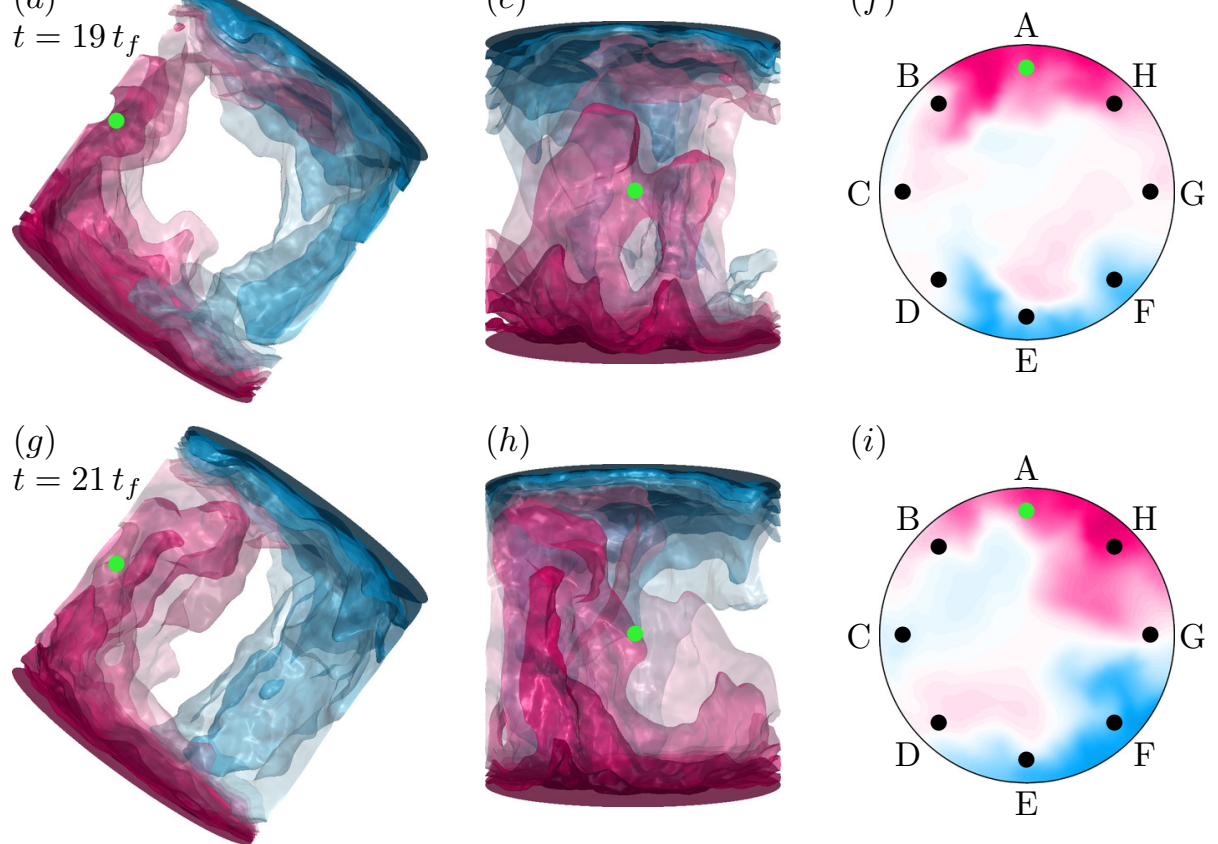

$(h)$

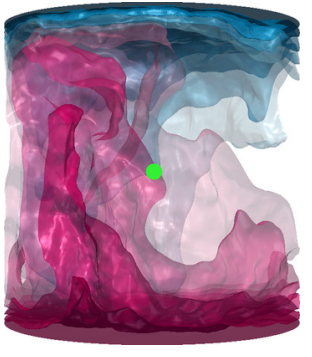

(i)

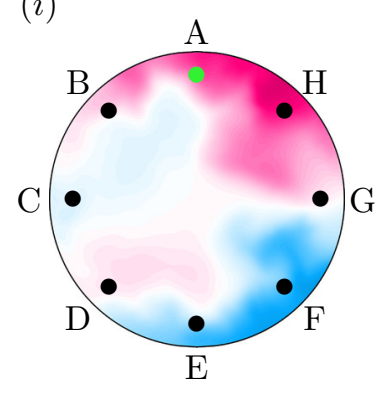

Figure 4.13: 3D side views $(a, d, g)$ and views orthogonal to the side views and to the cylinder axis $(b, e, h)$ of the temperature isosurfaces and the corresponding horizontal slices at the mid-height of the instantaneous temperature fields as seen from the cold plate $(c, f, i)$, which are obtained in the DNS for $R a=1.67 \times 10^{7}, \operatorname{Pr}=0.0094$ and the cell inclination angle $\beta=36^{\circ}$ at the times $(a, b, c)$ $t=17 t_{f},(d, e, f) t=19 t_{f},(g, h, i) t=21 t_{f}$. The dot (green online) marks the location A3. The here presented snapshots correspond to the times, marked in the figure 4.12 with the vertical lines. 


\begin{tabular}{rlr}
$n$ & $\theta_{\text {err }}$ & \multicolumn{1}{c}{$\delta_{\text {err }}$} \\
\hline 8 & $8.6^{\circ}$ & $12.2 \%$ \\
16 & $2.6^{\circ}$ & $3.6 \%$ \\
32 & $0.6^{\circ}$ & $0.8 \%$ \\
64 & $0.07^{\circ}$ & $0.1 \%$ \\
\hline
\end{tabular}

Table 4.5: Uncertainties $\theta_{\text {err }}=\left|\theta^{(n)}-\theta^{(512)}\right|$ and $\delta_{\text {err }}=\mid \delta^{(n)} / \delta^{(512)}-1$. $\mid$ that are caused by the limited number of probes $n$ while using the cosmethod to determine the phase $\theta_{i}$ and strength $\delta_{i}$ of the LSC. All values are averaged over the 5 circles and 400 snapshots of the DNS data for $R a=1.67 \times 10^{7}, \operatorname{Pr}=0.0094, \beta=0^{\circ}$.

Figure $4.12 \mathrm{~b}$ presents the evolution in time of the LSC phase $\theta_{1}$ in the circle 1 (close to the heated plate) and of the phase $\theta_{5}$ in circle 5 (close to the cold plate). These phases are anti-correlated, and the twisting of the LSC near the plates is maximal (at $t / t_{f}=19$ ) when the hot and the cold streams of the LSC in the central cross-section are located near the opposite sides of the cylinder sidewall.

In figure $4.12 \mathrm{c}$ the temporal evolution of the volume-averaged heat-flux vector parallel to the cylinder axis, $\left\langle\Omega_{z}\right\rangle_{\mathrm{V}}$, is presented. Again, a very strong relationship with the LSC twisting and sloshing is observed. The maximal values of $\left\langle\Omega_{z}\right\rangle_{\mathrm{V}}$ are obtained when the hot and cold LSC streams meet, thanks to sloshing, while the minimum value is obtained at the time periods when the LSC is strongly twisted.

In figure 4.13, the above described process, namely, the azimuthal movement of the hot and cold batches of fluid in the form of an oscillatory motion against each other, is illustrated with three-dimensional side views in two perpendicular directions. Additionally, the corresponding horizontal crosssections of the instantaneous temperature fields at the mid-height of the cylinder are presented there. In the supplementary movies to this paper, available at https://doi.org/10.1017/jfm.2019.935, the described dynamics of the LSC can be observed in detail.

Statistics of the large-scale circulation. In figure 4.14a the standard deviations of the phases $\theta_{i}$ in the circles $i=1,3$ and 5 are presented, while figure $4.14 \mathrm{~b}$ shows the corresponding time-averaged strengths of the LSC, $\left\langle\delta_{i}\right\rangle_{t}$, as they are obtained in the liquid-sodium measurements and DNS. The measurements show that the standard deviations of the phases $\theta_{1}$ (near the heated plate) and $\theta_{5}$ (near the cooled plate) are relatively large for small inclination angles, while being small for large inclination angles. There exist almost im- 

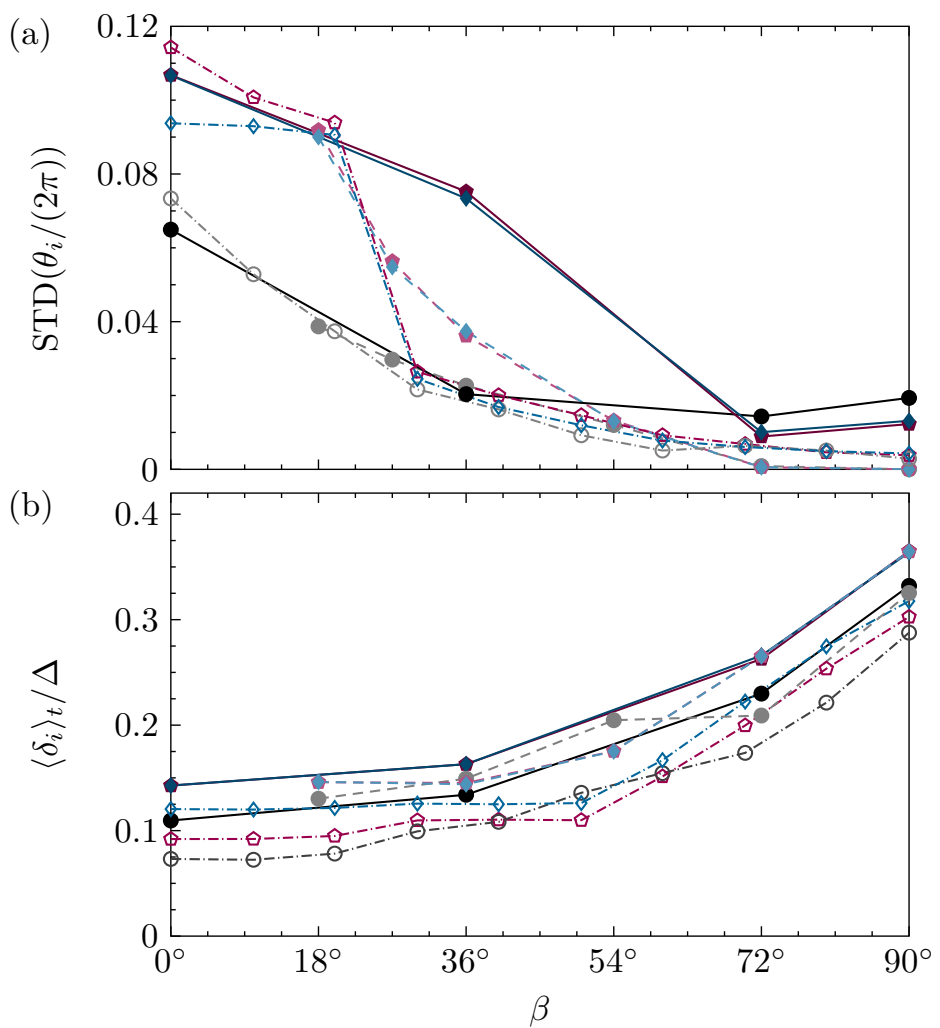

Figure 4.14: (a) Standard deviations of the phase angles $\theta_{i}$ in radians normalized by $2 \pi$ and $(b)$ the time-averaged strengths of the large scale circulation, $\left\langle\delta_{i}\right\rangle_{t}$, as obtained at the probe circle 1 (pink colour symbols and lines), circle 3 (grey colour) and circle 5 (blue colour) in the experiments (dash-dotted lines), the DNS for $R a=1.67 \times 10^{7}, \operatorname{Pr}=0.0094$ (solid lines) and DNS for $R a=10^{6}$, $\operatorname{Pr}=0.094$ (dash lines). 
mediate drops of $\theta_{1}(\beta)$ and $\theta_{5}(\beta)$ that happen between $\beta=20^{\circ}$ and $\beta=40^{\circ}$, which indicate a sharp transition between the twisting and sloshing modes of the LSC and the usual mode of the LSC, when it is not twisted and located basically in the central vertical cross-section along the axis of the cylindrical sample. The standard deviations of $\theta_{1}, \theta_{3}$ and $\theta_{5}$, obtained in the DNS, show generally a similar behaviour as those measured in the experiments, but due to only a few considered inclination angles in the DNS, it is impossible to resolve the sudden drop which is observed in the measurements. Also one should notice that the data in figure 4.14 are very sensitive to the time of statistical averaging, which is extremely short in the DNS compared to the experiment.

The results for the time-averaged strengths of the $\mathrm{LSC},\left\langle\delta_{i}\right\rangle_{t}$, obtained in the measurements and DNS (figure 4.14b) show similar trends. In the RBC case $\left(\beta=0^{\circ}\right)$, the LSC strength is small and grows smoothly with the inclination angle $\beta$. However, the mean strength of the LSC in the measurements is approximately $0.02 \Delta$, i.e. $2 \%$ of the temperature difference, weaker that the one in the liquid-sodium DNS. This is consistent with the lower Nusselt number, as well as the lower Rayleigh number of the experiments. Surprisingly, the liquid-sodium DNS data show a similar strength of the LSC as the data from the auxiliary DNS for the same $R a P r$.

Evaluating the LSC phase angle $\theta_{i}$ and strength $\delta_{i}$ using the cos method (Cioni et al., 1997) by fitting only 8 probes results in an uncertainty of approximately $8.6^{\circ}$ and $12.2 \%$, respectively. In table 4.5 we compare the mean deviation of the fit with a given number of probes $n$ compared to the case $n=512$. We find that the use of 32 probes is sufficient to reduce the uncertainty to below $1^{\circ}$ and $1 \%$, respectively. However, using only 8 probes in the experiment is acceptable, especially considering that every additional probe disturbs the flow.

\subsection{Conclusions}

In our complementary and cross-validating experimental and numerical studies, we have investigated inclined turbulent thermal convection in liquid sodium $(\operatorname{Pr} \approx 0.009)$ in a cylindrical container of aspect ratio one. The results of the conducted measurements, DNS and LES demonstrated a good quantitative agreement, and the uncertainty margin has been extensively discussed.

For the limiting cases of inclined convection, which are Rayleigh-Bénard convection (with the cell inclination angle $\beta=0^{\circ}$ ) and vertical convection $\left(\beta=90^{\circ}\right)$, we have experimentally studied the scaling relations of the mean heat flux (Nusselt number) with Rayleigh number, for $R a$ around $10^{7}$. The 
scaling exponents were found to be approximately 0.22 in both cases, but the absolute values of $N u$ are found to be larger in $\mathrm{VC}$, compared to those in $\mathrm{RBC}$. At the considered Rayleigh number of approximately $1.5 \times 10^{7}$, any inclination of the RBC cell generally leads to an increase of the mean heat flux. The maximal $N u$ is obtained, however, for a certain intermediate value of $\beta$ and may increase up to $29 \%$ (compared to the RBC case) for liquid sodium at the considered Rayleigh number.

It was proved, in particular, that the use of the cross-correlation time of the neighbouring temperature probes is a reliable tool to evaluate velocities during the temperature measurements in liquid sodium.

We showed (cf. tab. 5) that, using the cos method (4.20) to determine the phase $\theta_{i}$ and strength $\delta_{i}$ of the LSC with only 8 probes, one obtains reliable results with an uncertainty of approximately $8.6^{\circ}$ and $\pm 12.2 \%$, respectively.

Furthermore, we have found that for small Prandtl numbers there exists a similarity of IC flows of the same $R a P r$, due to the similar ratio, $t_{\kappa} / t_{f} \sim$ $\sqrt{R a P r}$, between the thermal diffusion time scale, $t_{\kappa}$, and the free-fall time scale, $t_{f}$. Since in small- $\operatorname{Pr}$ convective flows, the viscous diffusion time scale, $t_{\nu}$, is much larger than $t_{\kappa}$, the value of $R a \operatorname{Pr}$ determines basically the mean temperature and heat-flux distributions. The Nusselt numbers and the relative Reynolds numbers of the liquid-sodium DNS and axillary DNS at similar $\mathrm{Ra} P r$ are similar for any inclination of the convection cell with respect to the gravity vector. This property can be very useful for the investigation of, e.g., the scaling relations of $N u$ and $R e$ with $R a$ and $\operatorname{Pr}$ or of the mean temperature or heat-flux distributions, at extremely high $R a$ and/or extremely small $\mathrm{Pr}$.

For small inclination angles, the large-scale circulation exhibits complex dynamics, with twisting and sloshing modes and these modes strongly influence the instantaneous heat transport in the system $( \pm 44 \%$ of the mean heat transport). In a strongly twisted LSC, the volume-averaged vertical heat flux is minimal, and it is maximal, shortly after the LSC sloshing mode brought together the hot and cold streams of the LSC. Note that both modes are present in the flow simultaneously. Figures 4.12 and 4.13 and supplementary movies illustrate the studied LSC dynamics. Additional investigations will be needed to study the even more complex behaviour of the LSC in IC of low- $P r$ fluids in elongated containers with $L \gg D$.

Acknowledgements. This work is supported by the Priority Programme SPP 1881 Turbulent Superstructures of the Deutsche Forschungsgemeinschaft (DFG) under the grant Sh405/7. O.S. also thanks the DFG for the support under the grant Sh405/4 - Heisenberg 
fellowship. The authors acknowledge the Leibniz Supercomputing Centre (LRZ) for providing computing time and the Institute of Continuous Media Mechanics (ICMM UB RAS) for providing resources of the Triton supercomputer. 


\section{Summary \& Conclusions}

This work explored some of the fascinating dynamics of turbulent thermal convection in liquid metals by conducting direct numerical simulations and comparing some of them with experimental results. The highly resolved direct numerical simulations of turbulent thermal convection in liquid sodium are unprecedented, in the duration of collecting statistical data in combination with such a high Rayleigh number $\left(R a=1.67 \times 10^{7}\right)$ and low Prandtl number $(\operatorname{Pr}=0.0094)$. The focus of this work was set to three different aspects concerning natural turbulent thermal convection. First, the effect of the competition between buoyancy and shear, that arises when the RBC cell is tilted with respect to gravity, on the heat and momentum transport in the system and on the global flow structures. Second, the effect of lateral confinement of the fluid layer within slender cylindrical containers. These boundary conditions strongly affect the flow structures emerging in turbulent convection. Multi-roll flow states were identified and it was found, that they show some differences and some similarities to multi-roll flow states in high Prandtl number fluids. Third, the fluids of interest are liquid metals with Prandtl numbers less than one, while special attention was given to liquid sodium because it has one of the lowest Prandtl numbers $(\operatorname{Pr} \approx 0.009)$.

Inclined convection. A favourable combination of the Rayleigh number, Prandtl number, aspect ratio and inclination angle can lead to a large increase in heat transport compared to that in classical Rayleigh-Bénard convection. Among the studied cases, the highest relative increase of the heat transport was observed for $\operatorname{Ra}=10^{7}, \operatorname{Pr}=0.1$ and an inclination angle of $\beta=\pi / 4$ in an aspect ratio $1 / 5$ cylinder. In this case, the heat transport is increased by a factor of approximately 2.3 (Zwirner \& Shishkina, 2018).

Changes in the global flow structure were identified that lead to the significant increase of the heat transport. First, inside a geometrically confined, slender container there is a stronger enhancement of the large-scale circulation, which is caused by the shear flow in inclined convection. Additionally, the large-scale Reynolds number, $R e_{U}$, can be several times larger in inclined convection compared to RBC. Thus, it increases by a factor of about 4.5 at the optimal inclination angle compared to no inclination for the slender cylinder and $R a=10^{9}$ and $\operatorname{Pr}=1$. In the aspect ratio one cylinder, the large-scale Reynolds number increased only by a factor of 1.6, for the same Rayleigh and Prandtl numbers (Zwirner \& Shishkina, 2018).

Second, inside the slender cylinder with inclination, plumes stack onto each 
other forming plume columns that eventually reach and impinge on the opposite boundary layer. Since the optimal heat transport is always observed as soon as those plume columns span the whole cylinder, the interaction of the plumes with the opposite BL is certainly important for the heat transport enhancement. This impinging not only squeezes the thermal boundary layer and increases the temperature gradient along the cylinder axis close to the plate (which increases the heat transport), but also it enhances the large-scale circulation and with it, the capability of transferring the heat to the opposite plate (Zwirner \& Shishkina, 2018).

Third, usually at an inclination angle, larger than the optimal one, the flow becomes laminar-like or even steady. This transition to steady flow was observed for all parameters except for the combination of the highest Rayleigh number and the lowest Prandtl number for a slender cylinder of aspect ratio $\Gamma=1 / 5$. Furthermore, this transition to steady flow may shows also oscillatory states, which means, that the route to turbulence in inclined convection is similar to that in RBC. Moreover, this also implies, that turbulent heat transport is not necessarily more efficient than laminar heat transport. Additionally, we observed that for lower Prandtl number and/or larger aspect ratio, the optimal heat transport is reached at a larger value of $\beta / \pi$ (Zwirner \& Shishkina, 2018).

Since in a slender geometry the side walls strongly affect the flow, the scaling relations for convection between infinitely extended plates do not hold in confined convection. Another observation is that the symmetry is broken by the tilt of the cylinder and asymmetric flow fields emerge. For turbulent RBC inside the slender cylinder we did not find a prevailing large-scale circulation as in the aspect-ratio-one cell. However, the presence of the LSC is not that important in RBC, since the heat transport is about the same in the $\Gamma=1$ and $\Gamma=1 / 5$ cases. This is in conformity with previous results by Ciliberto et al. (1996). Important to note is, though, that in inclined convection inside small aspect ratio cylinders, the LSC appears to play a very important role for the heat transport. Thus, the data suggest that the highest increase in heat transport for a certain aspect ratio not only depends on the inclination angle but also on the Rayleigh number and Prandtl number (Zwirner \& Shishkina, 2018).

Multiple-roll flow states. The existence of single- and double-roll states in $\mathrm{RBC}$ were observed and it was confirmed that the double-roll state has indeed less efficient heat transport compared to the single-roll state $\left(\operatorname{Ra}=10^{6}, \operatorname{Pr}=\right.$ 
0.1 and $\Gamma=1 / 5)$. Advancing from this observation, also more than two rolls were found at slightly higher Rayleigh number $\left(R a=5 \times 10^{6}\right)$. The convective flow structure inside a slender cylinder (aspect ratio $\Gamma=1 / 5$ ) consists of a single large-scale circulation roll or up to four rolls on top of each other. The rolls do not necessarily have the same height and the more rolls there are, the more the heat transport is inhibited. This effect seems to be much stronger in low-Prandtl number fluids $(\operatorname{Pr}=0.1)$ compared to water $(\operatorname{Pr} \approx 5$, cf. Xi \& Xia (2008)) (Zwirner et al., 2020b).

The mechanism, that breaks up the single large-scale circulation roll was identified as the elliptical instability. This instability enhances the vertical component of vorticity $\left(\omega_{z}\right)$, which causes azimuthal flow and thus the twisting and breakup of the large-scale circulation. Moreover, the time and volume averaged exact relationships of the energy dissipation rates, the enstrophy and the Nusselt number almost hold instantaneously in time, only with a small time shift between the signals. This is also the main indicator of the elliptical instability, as the vertical component of the enstrophy $\left(\omega_{z}^{2}\right)$ increases with a short delay in time after the horizontal component of the enstrophy (measure of the strength of the LSC) increased. The most likely flow state was a tworoll structure, where the rolls are located on top of each other (Zwirner et al., $2020 b)$.

Convection in liquid sodium. In the complementary and cross-validating experimental and numerical studies, inclined turbulent thermal convection in liquid sodium $(\operatorname{Pr} \approx 0.009)$ in a cylindrical container of aspect ratio one was investigated. The results of the conducted measurements, DNS and LES demonstrated a good quantitative agreement, and the uncertainty margin has been extensively discussed (Zwirner et al., 2020a).

Since in the low- $\operatorname{Pr}$ convective flows, the viscous diffusion time scale $\left(t_{\nu}\right)$ is much larger than $t_{\kappa}$, the value of $R a \operatorname{Pr}$ determines basically the mean temperature and heat flux distributions (Zwirner et al., 2020a). The Nusselt numbers and the relative Reynolds numbers obtained in the liquid sodium DNS and in axillary DNS at similar $R a \operatorname{Pr}$ were found to be similar for any inclination of the convection cell with respect to the gravity vector (Zwirner et al., 2020a). This property can be very useful for the investigation of, e.g., the scaling relations of $N u$ and $R e$ with $R a$ and $P r$ or of the mean temperature or heat flux distributions for the combination of extremely high $R a$ and extremely low $\operatorname{Pr}$ (Zwirner et al., 2020a).

For small inclination angles, the large-scale circulation exhibits complex dy- 
namics, with twisting and sloshing modes and these modes strongly influence the instantaneous heat transport in the system $( \pm 44 \%$ of the mean heat transport). In a strongly twisted LSC, the volume-average vertical heat flux is minimal, and it is maximal, shortly after the LSC sloshing mode brought together the hot and cold streams of the LSC. Note that both modes are present in the flow simultaneously (Zwirner et al., 2020a).

Conclusion. The present work demonstrated the influence of the aspect ratio, small Prandtl numbers and inclination angle on the heat transport and flow structure in turbulent thermal convection. It was shown that the dynamics of the large-scale circulation, i.e. the twisting and sloshing, significantly effects the instantaneous heat transport. The flow structure responsible for the strong increase of the heat transport in slender, inclined cylinders in liquid metal convection are plume columns supported by the large-scale circulation, which impinge on the opposite thermal boundary layers. In confined RBC, multiple rolls can form on top of each other and affect the heat transport efficiency.

Altogether, this work explored the flow structures and their effect on the heat transport in inclined turbulent thermal convection of low-Prandtl number fluids. 


\section{References}

Ahlers, G., Brown, E., Araujo, F. F., Funfschilling, D., GrossMANn, S. \& Lohse, D. 2006 a Non-Oberbeck-Boussinesq effects in strongly turbulent Rayleigh-Bénard convection. J. Fluid Mech. 569, 409-446.

Ahlers, G., Brown, E. \& Nikolaenko, A. $2006 b$ The search for slow transients, and the effect of imperfect vertical alignment, in turbulent Rayleigh-Bénard convection. J. Fluid Mech. 557, 347-367.

Ahlers, G., Grossmann, S. \& Lohse, D. 2009 Heat transfer and large scale dynamics in turbulent Rayleigh-Bénard convection. Rev. Mod. Phys. 81, 503-537.

BAI, K., Ji, D. \& Brown, E. 2016 Ability of a low-dimensional model to predict geometry-dependent dynamics of large-scale coherent structures in turbulence. Phys. Rev. E 93, 023117.

Bailon-Cuba, J., Shishkina, O., Wagner, C. \& Schumacher, J. 2012 Low-dimensional model of turbulent mixed convection in a complex domain. Phys. Fluids 24, 107101.

BARs, M. Le \& Dizes, S. LE 2006 Thermo-elliptical instability in a rotating cylindrical shell. J. Fluid Mech. 563, 189-198.

Belmonte, A., Tilgner, A. \& Libchaber, A. 1995 Turbulence and internal waves in side-heated convection. Phys. Rev. E 51, 5681-5687.

Belyaev, I.A., L.G., Genin, Listratov, Ya.I., Melnikov, I.A., SviriDov, V.G., Sviridov, E.V., Ivochkin, Yu.P., Razuvanov, N.G. \& ShPANSKY, YU.S. 2013 Specific features of liquid metal heat transfer in a tokamak reactor. Magnetohydrodynamics 49, 177-190.

BÉNARD, H. 1900 Les tourbillons cellulairs dans une nappe liquide. Rev. Gén. Sciences Pure Appl. 11, 1261-1271, 1309-1328.

BÉNARD, H. 1901 Les tourbillons cellulairs dans une nappe liquide. Méthodes optiques d'observation et d'enregistrement. J. Phys. Théor. Appl. 10, 254-266.

Blasius, H. 1908 Grenzschichten in Flüssigkeiten mit kleiner Reibung. Z. Math. Phys. 56, 1-37.

Bodenschatz, E., Pesch, W. \& Ahlers, G. 2000 Recent developments in Rayleigh-Bénard convection. Annu. Rev. Fluid Mech. 32, 709-778.

Brown, E. \& Ahlers, G. 2006 Rotations and cessations of the large-scale circulation in turbulent Rayleigh-Bénard convection. J. Fluid Mech. 568, 351-386.

Brown, E. \& Ahlers, G. 2009 The origin of oscillations of the large-scale circulation of turbulent Rayleigh-Bénard convection. J. Fluid Mech. 638, 383-400.

Castaing, B., Rusaouën, E., Salort, J. \& Chillà, F. 2017 Turbulent heat transport regimes in a channel. Phys. Rev. Fluids 2, 062801.

Chen, F., Huai, X., Cai, J., Li, X. \& Meng, R. 2013 Investigation on the applicability of turbulent-Prandtl-number models for liquid lead-bismuth eutectic. Nuclear Engineering and Design 257, 128 - 133. 
Chen, Y.-M. \& Pearlstein, A. J. 1989 Stability of free-convection flows of variable-viscosity fluids in vertical and inclined slots. J. Fluid Mech. 198, 513-541.

Chillà, F., Rastello, M., Chaumat, S. \& Castaing, B. 2004 Long relaxation times and tilt sensitivity in Rayleigh-Bénard turbulence. Eur. Phys. J. B 40 (2), 223-227.

Chillà, F. \& Schumacher, J. 2012 New perspectives in turbulent Rayleigh-Bénard convection. Eur. Phys. J. E 35, 58.

Ching, E. S. C., Dung, O.-Y. \& Shishrina, O. 2017 Fluctuating thermal boundary layers and heat transfer in turbulent Rayleigh-Bénard convection. J. Stat. Phys. 167, 626-635.

Chong, K. L., Huang, S.-D., Kaczorowski, M. \& XiA, K.-Q. 2015 Condensation of coherent structures in turbulent flows. Phys. Rev. Lett. 115, 264503.

Chong, K. L., Wagner, S., Kaczorowski, M., Shishkina, O. \& Xia, K.-Q. 2018 Effect of Prandtl number on heat transport enhancement in Rayleigh-Bénard convection under geometrical confinement. Phys. Rev. Fluids 3, 013501.

Chong, K. L. \& XIA, K.-Q. 2016 Exploring the severely confined regime in Rayleigh-Bénard convection. J. Fluid Mech. 805, R4.

Christensen, U. R. \& Tilgner, A. 2004 Power requirement of the geodynamo from ohmic losses in numerical and laboratory dynamos. Nature 429, $169-171$.

Churchill, S. W. \& Chu, H. H. S. 1975 Correlating equations for laminar and turbulent free convection from a vertical plate. Int. J. Heat Mass Transfer 18, 1323-1329.

Ciliberto, S., Cioni, S. \& Laroche, C. 1996 Large-scale flow properties of turbulent thermal convection. Phys. Rev. E 54, R5901-R5904.

Cioni, S., Ciliberto, S. \& Sommeria, J. 1997 Strongly turbulent Rayleigh-Bénard convection in mercury: Comparison with results at moderate Prandtl number. J. Fluid Mech. 335, 111-140.

Daniels, K. E., Plapp, B. B. \& Bodenschatz, E. 2000 Pattern formation in inclined layer convection. Phys. Rev. Lett. 84, 5320-5323.

Daniels, K. E., Wiener, R. J. \& Bodenschatz, E. 2003 Localized transverse bursts in inclined layer convection. Phys. Rev. Lett. 91, 114501.

DeardorfF, J. W. 1970 A numerical study of three-dimensional turbulent channel flow at large Reynolds numbers. J. Fluid Mech. 41, 453-480.

Dropkin, D. \& Somerscales, E. 1965 Heat transfer by natural convection in liquids confined by two parallel plates which are inclined at various angles with respect to the horizontal. J. Heat Transfer. 87 (1), 77-82.

Ferziger, J. H. \& Perić, M. 2002 Computational Methods for Fluid Dynamics, 3rd edn. Springer.

FletcheR, R. 1976 Conjugate gradient methods for indefinite systems. In Numerical Analysis (ed. G. Alistair Watson), 506, 73-89. Springer Berlin Heidelberg. 
Frick, P., Khalilov, R., Kolesnichenko, I., Mamykin, A., Pakholkov, V., Pavlinov, A. \& Rogozhkin, S. A. 2015 Turbulent convective heat transfer in a long cylinder with liquid sodium. Europhys. Lett. 109, 14002.

Fujis, T., Takeuchi, M., FujiI, M., Suzaki, K. \& Uehar, H. 1970 Experiments on natural-convection heat transfer from the outer surface of a vertical cylinder to liquids. Int. J. Heat Mass Transfer 13, 753-770.

Fujimura, K. \& Kelly, R. E. 1993 Mixed mode convection in an inclined slot. J. Fluid Mech. 246, 545-568.

Funfschilling, D. \& Ahlers, G. 2004 Plume motion and large-scale dynamics in a cylindrical Rayleigh-Bénard cell. Phys. Rev. Lett. 92, 194502.

Funfschilling, D., Brown, E. \& Ahlers, G. 2008 Torsional oscillations of the large-scale circulation in turbulent Rayleigh-Bénard convection. J. Fluid Mech. 607, 119-139.

Garaud, P. 2018 Double-diffusive convection at low Prandtl number. Ann. Rev. Fluid Mech. 50 (1), 275-298.

George, W. K. \& CAPP, S. P. 1979 A theory for natural convection turbulent boundary layers next to heated vertical surfaces. Int. J. Heat Mass Transfer 22, 813-826.

Glazier, J. A., Segawa, T., A., Naert \& M., Sano 1999 Evidence against 'ultrahard' thermal turbulence at very high Rayleigh numbers. $\mathrm{Na}$ ture 398, 307-310.

Green, G., Vlaykov, D. G., Mellado, J. P. \& Wilczek, M. 2020 Resolved energy budget of superstructures in Rayleigh-Bénard convection. J. Fluid Mech. 887, A21.

Grossmann, S. \& Lohse, D. 2000 Scaling in thermal convection: A unifying theory. J. Fluid Mech. 407, 27-56.

Grossmann, S. \& Lohse, D. 2001 Thermal convection for large Prandtl numbers. Phys. Rev. Lett. 86, 3316-3319.

Grossmann, S. \& Lohse, D. 2011 Multiple scaling in the ultimate regime of thermal convection. Phys. Fluids 23, 045108.

Guo, S.-X., Zhou, S.-Q., Cen, X.-R., Qu, L., Lu, Y.-Z., Sun, L. \& SHANG, X.-D. 2015 The effect of cell tilting on turbulent thermal convection in a rectangular cell. J. Fluid Mech. 762, 273-287.

Hanasoge, S., Gizon, L. \& Sreenivasan, K. R. 2016 Seismic sounding of convection in the sun. Ann. Rev. Fluid Mech. 48, 191-217.

He, X., Bodenschatz, E. \& Ahlers, G. 2016 Azimuthal diffusion of the large-scale-circulation plane, and absence of significant non-Boussinesq effects, in turbulent convection near the ultimate-state transition. J. Fluid Mech. 791, R3.

Heinzel, A., Hering, W., Konys, J., Marocco, L., Litfin, K., Mueller, G., Pacio, J., Schroer, C., Stieglitz, R., Stoppel, L., Weisenburger, A. \& Wetzel, T. 2017 Liquid metals as efficient hightemperature heat-transport fluids. Energy Technology 5, 1026-1036. 
Horanyi, S., Krebs, L. \& Müller, U. 1999 Turbulent Rayleigh-Bénard convection low Prandtl-number fluids. Int. J. Heat \& Mass Transfer 42, 3983-4003.

Horn, S. \& Schmid, P. J. 2017 Prograde, retrograde, and oscillatory modes in rotating Rayleigh-Bénard convection. J. Fluid Mech. 831, 182-211.

Horn, S. \& Shishkina, O. 2015 Toroidal and poloidal energy in rotating Rayleigh-Bénard convection. J. Fluid Mech. 762, 232-255.

Horn, S., Shishkina, O. \& Wagner, C. 2013 On non-OberbeckBoussinesq effects in three-dimensional Rayleigh-Bénard convection in glycerol. J. Fluid Mech. 724, 175-202.

HuAng, L. \& El-GenK, M. S. 1994 Heat transfer of an impinging jet on a flat surface. International Journal of Heat and Mass Transfer 37 (13), $1915-1923$.

Huang, S.-D., Kaczorowski, M., Ni, R. \& Xia, K.-Q. 2013 Confinement induced heat-transport enhancement in turbulent thermal convection. Phys. Rev. Lett. 111, 104501.

IssA, R. 1986 Solution of the implicitly discretised fluid flow equations by operator-splitting. J. Comput. Phys. 62, 40-65.

Jambunathan, K., Lai, E., Moss, M. A. \& Button, B. L. 1992 A review of heat transfer data for single circular jet impingement. Int. J. Heat Es Fluid Flow 13 (2), 106-115.

Kerswell, R. R. 2002 Elliptical instability. Annu. Rev. Fluid Mech. 34, 83-113.

Khalilov, R., Kolesnichenko, I., Pavlinov, A., Mamykin, A., ShesTAKOV, A. \& FRICK, P. 2018 Thermal convection of liquid sodium in inclined cylinders. Phys. Rev. Fluids 3, 043503.

King, E. M. \& Aurnou, J. M 2013 Turbulent convection in liquid metal with and without rotation. Proc. Natl. Acad. Sci. 110, 6688-6693.

KIs, P. \& Herwig, H. 2012 The near wall physics and wall functions for turbulent natural convection. Int. J. Heat Mass Transfer 55, 2625-2635.

Kolesnichenko, I., Khalilov, R., Teimurazov, A. \& Frick, P. 2017 On boundary conditions in liquid sodium convective experiments. J. Physics: Conference Series 891 (1).

Kolesnichenko, I.V., Mamykin, A. D., Pavlinov, A. M., Pakholkov, V. V., Rogozhinin, S. A., Frick, P. G., Khalilov, R. I. \& ShepELEV, S. F. 2015 Experimental study on free convection of sodium in a long cylinder. Thermal Engineering 62, 414-422.

Kooij, G. L., Botchev, M. A., Frederix, E. M.A., Geurts, B. J., Horn, S., Lohse, D., van der Poel, E. P., Shishkina, O., Stevens, R. J. A. M. \& Verzicco, R. 2018 Comparison of computational codes for direct numerical simulations of turbulent Rayleigh-Bénard convection. Comp. Fluids 166, 1-8.

Kraichnan, R. 1962 Turbulent thermal convection at arbitrary Prandtl number. Phys. Fluids 5, 1374-1389. 
Krug, D., Lohse, D. \& Stevens, R. J. A. M. 2020 Coherence of temperature and velocity superstructures in turbulent Rayleigh-Bénard flow. $J$. Fluid Mech. 887, A2.

Kunnen, R. P. J., Stevens, R. J. A. M., Overkamp, J., Sun, C., van Heijst, G. F. \& Clercx, H. J. H. 2011 The role of Stewartson and Ekman layers in turbulent rotating Rayleigh-Bénard convection. J. Fluid Mech. 688, 422-442.

Landau, L. D. \& Lifshitz, E. M. 1987 Fluid Mechanics, 2nd edn., Course of Theoretical Physics, vol. 6. Butterworth Heinemann.

Landman, M. J. \& Saffman, P. G. 1987 The threedimensional instability of strained vortices in a viscous fluid. Phys. Fluids 30 (8), 2339-2342.

Langebach, R. \& Haberstroh, Ch. 2014 Natural Convection in Inclined Pipes - A New Correlation for Heat Transfer Estimations. In Advances in Cryogenic Engineering (ed. J.G. Weisend et al. ), AIP Conf. Proc., vol. 1573, pp. 1504-1511.

Leweke, T., Dizes, S. Le \& Williamson, C. H. K. 2016 Dynamics and instabilities of vortex pairs. Annual Rev. Fluid Mech. 48 (1), 507-541.

Leweke, T. \& Williamson, C. H. K. 1998 Cooperative elliptic instability of a vortex pair. J. Fluid Mech. 360, 85-119.

Lohse, D. \& XIA, K.-Q. 2010 Small-scale properties of turbulent RayleighBénard convection. Annu. Rev. Fluid Mech. 42, 335-364.

LORD RAYLEIGH 1916 On convection currents in a horizontal layer of fluid, when the higher temperature is on the under side. Phil. Mag. 32, 529-546.

Lorenz, H. H. 1934 Die Wärmeübertragung von einer ebenen, senkrechten Platte an öl bei natürlicher Konvektion. Zs. f. tech. Phys. 9 (15), 362-366.

Lorenzani, S. \& Tilgner, A. 2003 Inertial instabilities of fluid flow in precessing spheroidal shells. J. Fluid Mech. 492, 363-379.

Malkus, M. V. R. 1954 The heat transport and spectrum of thermal turbulence. Proc. R. Soc. London A 225, 196-212.

Mamykin, A., Frick, P., Khalilov, R., Kolesnichenko, I., Pakholkov, V., Rogozhkin, S. \& Vasiliev, A. 2015 Turbulent convective heat transfer in an inclined tube with liquid sodium. Magnetohydrodynamics 51, 329-336.

Mamykin, A., Kolesnichenko, I., Pablinov, A. M. \& Khalilov, R. 2018 Large scale circulation in turbulent Rayleigh-Bénard convection of liquid sodium in cylindrical cell. J. Phys.: Conf. Ser. 1128, 012019.

Mandrykin, S. D. \& Teimurazov, A. S. 2019 Turbulent convection of liquid sodium in an inclined cylinder of unit aspect ratio. J. Appl. Mech. Tech. Phys. 60 (7), (in press).

McDonald, J. S. \& Connolly, T. J. 1960 Investigation of Natural Convection Heat Transfer in Liquid Sodium. Nuclear Science and Engineering 8 (5), 369-377.

Mishra, P. K., De, A. K., Verma, M. K. \& Eswaran, V. 2011 Dynamics of reorientations and reversals of large-scale flow in Rayleigh-Bénard convection. J. Fluid Mech. 668, 480-499. 
NG, C. S., Ooi, A., Lohse, D. \& Chung, D. 2015 Vertical natural convection: application of the unifying theory of thermal convection. J. Fluid Mech. 764, 0349-361.

NG, C. S., Ooi, A., Lohse, D. \& Chung, D. 2017 Changes in the boundary-layer structure at the edge of the ultimate regime in vertical natural convection. J. Fluid Mech. 825, 550-572.

NG, C. S., Ooi, A., Lohse, D. \& Chung, D. 2018 Bulk scaling in wallbounded and homogeneous vertical natural convection. J. Fluid Mech. 841, $825-850$.

Niemela, J. J., Skrbek, L., Sreenivasan, K. R. \& Donnely, R. J. 2000 Turbulent convection at very high Rayleigh numbers. Nature 404, 837-841.

Onea, A., Hering, W., Lux, M. \& Stieglitz, R. 2017 a Numerical optimization of cold trap designs for the Karlsruhe Sodium Laboratory. Int. J. Heat Mass Transfer 113, 984-999.

Onea, A., Perez-Martin, S., Jaeger, W., Hering, W. \& Stieglitz, R. $2017 b$ Liquid metals as heat transfer fluids for science and technology, 1st edn. Advances in New Heat Transfer Fluids. From Numerical to Experimental Techniques, CRC Press.

Pandey, A., Scheel, J. D. \& Schumacher, J. 2018 Turbulent superstructures in Rayleigh-Bénard convection. Nat. Commun. 9, 2118.

van der Poel, E. P., Stevens, R. J. A. M. \& Lohse, D. 2011 Connecting flow structures and heat flux in turbulent Rayleigh-Bénard convection. Phys. Rev. E 84, 045303(R).

van der Poel, E. P., Stevens, R. J. A. M., Sugiyama, K. \& Lohse, D. 2012 Flow states in two-dimensional Rayleigh-Bénard convection as a function of aspect ratio and Rayleigh number. Phys. Fluids 24, 085104.

Prabhakaran, P., Weiss, S., Krekhov, A., Pumir, A. \& BodenSChatz, E. 2017 Can hail and rain nucleate cloud droplets? Phys. Rev. Lett. 119, 128701.

PrandTL, L. 1905 Über Flüssigkeitsbewegung bei sehr kleiner Reibung. In Verhandlungen des III. Int. Math. Kongr., Heidelberg, 1904, pp. 484-491. Teubner.

Riedinger, X., Tisserand, J.-C., Seychelles, F., Castaing, B. \& Chillá, F. 2013 Heat transport regimes in an inclined channel. Phys. Fluids 25, 015117.

Roche, P.-E., Gauthier, F., Kaiser, R. \& Salort, J. 2010 On the triggering of the ultimate regime of convection. New J. Phys. 12 (8), 085014.

Rossby, T. H. 1969 A study of Bénard convection with and without rotation. J. Fluid Mech. 36, 309-335.

Saunders, O. A. 1939 Natural convection in liquids. Proc. Roy. Soc. (London) A 172 (948), 55-71.

Scheel, J. D. \& Schumacher, J. 2016 Global and local statistics in turbulent convection at low Prandtl numbers. J. Fluid Mech. 802, 147-173. 
Scheel, J. D. \& Schumacher, J. 2017 Predicting transition ranges to fully turbulent viscous boundary layers in low Prandtl number convection flows. Phys. Rev. Fluids 2, 123501.

Schlichting, H. \& Gersten, K. 2000 Boundary-Layer Theory, 8th edn. Springer.

Schmidt, E. \& Beckmann, W. 1930 Das Temperatur- und Geschwindigskeitsfeld vor einer Wärme abgebenden senkrechten Platte bei natürlicher Konvektion. Forsch. Ing.-Wes. (Techn. Mech. Thermodyn.) 1, 341-349, 391-406.

Schumacher, J., Bandaru, V., Pandey, A. \& Scheel, J. D. 2016 Transitional boundary layers in low-Prandtl-number convection. Phys. Rev. Fluids 1, 084402.

Schumacher, J., Götzfried, P. \& Scheel, J. 2015 Enhanced enstrophy generation for turbulent convection in low-Prandtl-number fluids. Proc. National Academy of Sciences 112 (31), 9530-9535.

ShishkinA, O. 2016 Momentum and heat transport scalings in laminar vertical convection. Phys. Rev. E 93, 051102(R).

Shishkina, O., Emran, M., Grossmann, S. \& Lohse, D. 2017 Scaling relations in large-Prandtl-number natural thermal convection. Phys. Rev. Fluids 2, 103502.

Shishrina, O., Grossmann, S. \& Lohse, D. 2016 a Heat and momentum transport scalings in horizontal convection. Geophys. Res. Lett. 43, 12191225 .

ShishkinA, O. \& Horn, S. 2016 Thermal convection in inclined cylindrical containers. J. Fluid Mech. 790, R3.

Shishkina, O., Horn, S., Wagner, S. \& Ching, E. S. C. 2015 Thermal boundary layer equation for turbulent Rayleigh-Bénard convection. Phys. Rev. Lett. 114, 114302.

Shishkina, O., Stevens, R. J. A. M., Grossmann, S. \& Lohse, D. 2010 Boundary layer structure in turbulent thermal convection and its consequences for the required numerical resolution. New J. Phys. 12, 075022.

Shishkina, O. \& Wagner, S. 2016 Prandtl-number dependence of heat transport in laminar horizontal convection. Phys. Rev. Lett. 116, 024302.

Shishkina, O., Weiss, S. \& Bodenschatz, E. $2016 b$ Conductive heat flux in measurements of the Nusselt number in turbulent Rayleigh-Bénard convection. Phys. Rev. Fluids 1, 062301(R).

Shraiman, B. I. \& Siggia, E. D. 1990 Heat transport in high-Rayleighnumber convection. Phys. Rev. A 42, 3650-3653.

Spiegel, E. A. 1962 Thermal turbulence at very small Prandtl number. J. Geophys. Res. 67, 3063-3070.

Stevens, R. J. A. M., Blass, A., Zhu, X., Verzicco, R. \& Lohse, D. 2018 Turbulent thermal superstructures in Rayleigh-Bénard convection. Phys. Rev. Fluids 3, 041501. 
Stevens, R. J. A. M., Clercx, H. J. H. \& Lohse, D. 2011 Effect of plumes on measuring the large scale circulation in turbulent RayleighBénard convection. Phys. Fluids 23, 095110.

Stevens, R. J. A. M., van der Poel, E. P., Grossmann, S. \& Lohse, D. 2013 The unifying theory of scaling in thermal convection: The updated prefactors. J. Fluid Mech. 730, 295-308.

Sun, C., XI, H.-D. \& XIA, K.-Q. 2005 Azimuthal symmetry, flow dynamics, and heat transport in turbulent thermal convection in a cylinder with an aspect ratio of 0.5. Phys. Rev. Lett. 95, 074502.

Takeshita, T., Segawa, T., Glazier, J. A. \& Sano, M. 1996 Thermal turbulence in mercury. Phys. Rev. Lett. 76, 1465-1468.

Teimurazov, A. \& Frick, P. 2017 Thermal convection of liquid metal in a long inclined cylinder. Phys. Rev. Fluids 2, 113501.

Teimurazov, A., Frick, P. \& Stefani, F. 2017 Thermal convection of liquid metal in the titanium reduction reactor. IOP Conference Series: $M a-$ terials Science and Engineering 208, 012044.

Vasil'ev, A. Yu., Kolesnichenko, I. V., Mamykin, A. D., Frick, P. G., Khalilov, R. I., Rogozhkin, S. A. \& Pakholkov, V. V. 2015 a Turbulent convective heat transfer in an inclined tube filled with sodium. Technical Physics 60, 1305-1309.

Vasil'ev, A. Yu., Kolesnichenko, I. V., Mamykin, A. D., Frick, P. G., Khalilov, R. I., Rogozhkin, S. A. \& Pakholkov, V. V. $2015 b$ Turbulent convective heat transfer in an inclined tube filled with sodium. Technical Physics 60, 1305-1309.

Versteegh, T. A. M. \& Nieuwstadt, F. T. M. 1999 A direct numerical simulation of natural convection between two infinite vertical differentially heated walls scaling laws and wall functions. Int. J. Heat Mass Transfer 42, 3673-3693.

Verzicco, R \& Camussi, R 1997 Transitional regimes of low-Prandtl thermal convection in a cylindrical cell. Phys. Fluids 9 (5), 1287-1295.

Vogt, T., Horn, S., Grannan, A. M. \& Aurnou, J. M. 2018 Jump rope vortex in liquid metal convection. PNAS 115, 12674-12679.

Wagner, S. \& Shishkina, O. 2013 Aspect ratio dependency of RayleighBénard convection in box-shaped containers. Phys. Fluids 25, 085110.

WAGNer, S. \& Shishkina, O. 2015 Heat flux enhancement by regular surface roughness in turbulent thermal convection. J. Fluid Mech. 763, 109-135.

Wagner, S., Shishkina, O. \& Wagner, C. 2012 Boundary layers and wind in cylindrical Rayleigh-Bénard cells. J. Fluid Mech. 697, 336-366.

WALEFFE, F. 1990 On the threedimensional instability of strained vortices. Phys. Fluids 2 (1), 76-80.

WEI, P. \& XIA, K.-Q. 2013 Viscous boundary layer properties in turbulent thermal convection in a cylindrical cell: the effect of cell tilting. J. Fluid Mech. 720, 140-168.

Weiss, S. \& Ahlers, G. 2013 Effect of tilting on turbulent convection: cylindrical samples with aspect ratio $\gamma=0.50$. J. Fluid Mech. 715, 314-334. 
Weiss, S., He, X., Ahlers, G., Bodenschatz, E. \& Shishkina, O. 2018 Bulk temperature and heat transport in turbulent Rayleigh-Bénard convection of fluids with temperature-dependent properties. J. Fluid Mech. 851, 374-390.

Weller, H. G., H., G. Tabor, Jasak \& Fureby, C. 1998 A tensorial approach to computational continuum mechanics using object-oriented techniques. Computers in Physics 12, 620-631.

XI, H.-D. \& XIA, K.-Q. 2007 Cessations and reversals of the large-scale circulation in turbulent thermal convection. Phys. Rev. E 76, 036301.

XI, H.-D. \& XIA, K.-Q. 2008 Flow mode transitions in turbulent thermal convection. Phys. Fluids 20, 055104.

Xi, H.-D., Zhou, Q. \& XiA, K.-Q. 2006 Azimuthal motion of the mean wind in turbulent thermal convection. Phys. Rev. E 73, 056312.

Xı, H.-D., Zhou, S.-Q., Zhou, Q., Chan, T. S. \& Xia, K.-Q. 2009 Origin of the temperature oscillations in turbulent thermal convection. Phys. Rev. Lett. 102, 044503.

XIE, H. Q., WeI, P. \& XIA, .K-Q. 2013 Dynamics of the large-scale circulation in high-prandtl-number turbulent thermal convection. J. Fluid Mech. 717, 322-346.

Yu, H., LI, N. \& ECKE, R. E. 2007 Scaling in laminar natural convection in laterally heated cavities: Is turbulence essential in the classical scaling of heat transfer? Phys. Rev. E 76, 026303.

Zhang, X., van Gils, D. P. M., Horn, S., Wedi, M., Zwirner, L., Ahlers, G., Ecke, R. E., Weiss, S., Bodenschatz, E. \& ShishkINA, O. 2020 Boundary zonal flow in rotating turbulent Rayleigh-Bénard convection. Phys. Rev. Lett. 124, 084505.

Zhang, X. \& Zikanov, O. 2014 Mixed convection in a horizontal duct with bottom heating and strong transverse magnetic field. J. Fluid Mech. 757, $33-56$.

Zhilin, V.G., Sviridov, V.G., Razuvanov, N.G., Ivochkin, Y.P., Listratov, Ya.I., Sviridov, E.V. \& Belyaev, I.A. 2009 Influence of secondary flows on the liquid metal heat transfer in a horizontal pipe with nonuniform heating in presence of a magnetic field. Thermal Processes in Engineering 1, 199-203.

Zhou, Q., Xi, H.-D., Zhou, S.-Q., Sun, C. \& XiA, K.-Q. 2009 Oscillations of the large-scale circulation in turbulent Rayleigh-Bénard convection: The sloshing mode and its relationship with the torsional mode. J. Fluid Mech. 630, 367-390.

Zhou, Q. \& XIA, K.-Q. 2010 Measured instantaneous viscous boundary layer in turbulent Rayleigh-Bénard convection. Phys. Rev. Lett. 104, 104301.

Zimin, V. D., Frik, P. G. \& Shaidurov, V. G. 1982 Turbulent convection in a cubic cell heated simultaneously on the side and the bottom. Fluid Dynamics 17, 286-290. 
Zürner, T., Schindler, F., Vogt, T., Eckert, S. \& Schumacher, J. 2019 Combined measurement of velocity and temperature in liquid metal convection. J. Fluid Mech. 876, 1108-1128.

Zwirner, L., Khalilov, R., Kolesnichenko, I., Mamykin, A., Mandrykin, S., Pavlinov, A., Shestakov, A., Teimurazov, A., Frick, P. \& ShishkinA, O. 2020a The influence of the cell inclination on the heat transport and large-scale circulation in liquid metal convection. $J$. Fluid Mech. 884, A18.

Zwirner, L. \& Shishkina, O. 2018 Confined inclined thermal convection in low-Prandtl-number fluids. J. Fluid Mech. 850, 984-1008.

Zwirner, L., Tilgner, A. \& Shishkina, O. $2020 b$ Elliptical Instability and Multi-Roll Flow Modes of the Large-scale Circulation in Confined Turbulent Rayleigh-Bénard Convection, arXiv: 2002.06951. 